\title{
Engineered Living Materials: Prospects and Challenges for Using Biological Systems to Direct the Assembly of Smart Materials
}

\author{
Dr. Peter Q. Nguyen, Noémie-Manuelle Dorval Courchesne [Prof.], Anna Duraj-Thatte Dr., \\ Pichet Praveschotinunt, and Neel S. Joshi ${ }^{*}$ [Prof.] \\ School of Engineering and Applied Sciences, Wyss Institute for Biologically Inspired Engineering, \\ Harvard University, Cambridge, MA 02138, USA
}

\begin{abstract}
Vast potential exists for the development of novel, engineered platforms that manipulate biology for the production of programmed advanced materials. Such systems would possess the autonomous, adaptive, and self-healing characteristics of living organisms but would be engineered with the goal of assembling bulk materials with designer physicochemical or mechanical properties, across multiple length scales. Early efforts towards such Engineered Living Materials (ELMs) are reviewed here, with an emphasis on the engineered bacterial systems, living composite materials which integrate inorganic components, successful examples of large-scale implementation, and production methods. In addition, a conceptual exploration of the fundamental criteria of ELM technology and its future challenges is presented. Cradled within the rich intersection of synthetic biology and self-assembling materials, the development of ELM technologies will allow us to leverage the power of biology to grow complex structures and objects using a palette of bionanomaterials.
\end{abstract}

\section{Keywords}

Engineered Living Materials; Synthetic Biology; Biomaterials; Smart Materials; Self-healing

\section{Introduction}

Next generation advanced materials must include 'smart' functional properties that surpass existing capabilities, such as adaptation to environmental cues, the ability to dynamically switch between different material states, and self-healing capabilities. Materials with many of these properties have already been developed by living systems over the last $\sim 4.5$ billion years, as they have created biological structures for the modification of their environment and survival. From a materials standpoint, cells can be considered as nanomaterials factories that constantly sense their environment, draw from a plethora of energy sources and simplistic molecular building blocks, refashion these molecules into new structurally and functionally more complex materials, and maintain these materials over time. Given the 
amazing complexity of materials that biology is capable of producing, it is no surprise that bioinspired approaches to the design and engineering of materials is a fertile area of exploration. A core strategy that living systems employ is the use of biomolecular selfassembly to create large structures from molecular building blocks that have evolved to associate in a precise manner. Nowhere is this more apparent than the amazing accuracy of the self-assembling material employed by living systems for long-term information storage DNA. Thus, robust bottom-up fabrication methods are a fundamental aspect of biological materials. Much effort in the past few decades has focused on unravelling the mechanisms and regulation of biological self-assembly systems. As we have gleaned these core principles, they have been successfully applied in engineering efforts, creating artificial selfassembling materials composed of peptides, proteins, DNA, and carbohydrates. However, many of these engineered materials utilize biomolecular building blocks under highly controlled conditions - they are often purified and assembled in vitro. Unfortunately, many of the unique properties of living systems are lost under such conditions. A parallel engineering effort that has occurred in the past decade is Synthetic Biology, which focuses on applying knowledge of genetic regulation and biological systems towards the systematic development of forward-engineered artificial biological circuits.

A rich area has blossomed at the interface of self-assembling biomaterials and synthetic biology. Broadly, this area can be classified as biohybrid materials and encompasses any composite material that has a biologically-derived component and a synthetic component. The biological component may be purified biomolecules, such as proteins or DNA, or living cells. The synthetic component could be organic or inorganic polymers, minerals, ceramics, or even metals. Examples of such biohydrid materials/devices include cells that are spatially embedded or encapsulated in polymeric structures, such as stimuli-responsive materials composed of cells covalently linked with polymers ${ }^{[1]}$, microfluidic devices recapitulating complex organ-level systems ${ }^{[2-6]}$, propulsion of microparticles using attached bacteria ${ }^{[7]}$, wearables harboring genetically engineered cells ${ }^{[8]}$, encapsulated cells in fibers ${ }^{[9-11]}$ or solgels $^{[12,13]}$, and optically positioned cells on 3D microscaffold structures ${ }^{[14]}$. However, in these examples of biohybrid materials, biological component, whether it is biopolymeric or includes living cells, is just one element of the engineered structure and does not actively create or modulate the bulk structure of the material. This can be contrasted with actual biological systems, in which cells not only produce the material through a combination of organized self-replication and biopolymer synthesis, but also modify its properties through context-dependent active processes over its lifetime.

Here, we provide a comprehensive review of an emerging area within biohybrid materials, Engineered Living Materials (ELMs). We define ELMs as engineered materials composed of living cells that form or assemble the material itself, or modulate the functional performance of the material in some manner (Figure 1). A key difference between ELMs and other biohybrid devices is that in ELMs, the living cells act as materials factories, drawing upon energy feedstocks from their environment to create biopolymeric building blocks and direct the formation of, or maintain, the desired material. The engineered aspect of an ELM may take on a variety of forms, such as genetic engineering of the material components or simply spatial/mechanical engineering to restrict or position the cells. The ELM may be composed predominantly of the cellular biomass or could secrete materials that form a large part of the 
structure (such as in biofilms). Furthermore, ELMs may be designed to integrate other inorganic polymers, particles, or scaffolds into the material in some way as part of the material assembly process. Unlike most other self-assembled biomaterials that utilize purified components, ELM should be able to robustly direct the formation of the material in complex conditions containing a multitude of components, such as nutrient feedstocks or metabolic wastes. Because they are living, ELMs may also allow for significant programmed stimuli-responsive changes over the viable lifetime of the cellular components. If desired, an ELM can, at a particular juncture, be processed to remove or kill the living cells, retaining the assembled materials without concern for continued maintenance or potential biohazard threats of the cells.

The bioinspiration for ELMs can be found throughout nature, in how living systems manipulate and reshape the environment around them for their own gain (Figure 2). Consider a seed that has been honed by eons of genetic evolution, to contain all the necessary information within it to build nanomachines that harvest the promethean energy from the Sun. This harnessed energy is used to power the process of stitching together gas molecules from the atmosphere into larger molecules that form more cells and an extraordinary large-scale assembly of extracellular materials, cellulose and lignin. Throughout this structure building progression, which may occur over the course of decades, the embedded living cells continually orchestrate the organization of new nanoscale building blocks that ultimately enable the adoption of macroscale morphologies and functions to optimally respond to the changing environment. Trees, and the wood they produce, are an amazing example of the complex materials that biology is capable of generating. Such ability to encode for a rich palate of functional materials, drawing from diverse sources of energy and matter in the environment, directing the precise assembly of complex structures throughout multiple length scales, and adapting to the environment in a spatial and temporal manner are the key endeavors of ELM research.

In this review, we attempt to survey engineering efforts which can be considered ELM systems, based on the criteria described above. Although there are many overlapping biohybrid engineering efforts that possess some aspects of ELMs, we felt it would be prudent to restrict our exploration to technologies that at least conform to the most fundamental aspect: the living cells must fabricate or direct the assembly of the bulk material. It is arguably true that many tissue engineering efforts meet the criteria outlined above. However, as tissue engineering is a broad and well established field with many existing reviews and entire journals devoted to its coverage, we will focus here on ELM efforts targeted toward applications not related to regenerative medicine. As bacteria are the most genetically tractable organisms and are the workhorses of choice for synthetic biology, much ELM research has been undertaken in bacterial systems that generate extracellular matrix (ECM) materials. In Section 1, we describe ongoing efforts at designing ELMs based on the engineering of natural ECM biopolymer components. Next, we explore composite ELMs, in which the cells are engineered to manipulate and incorporate external elements or molecules into the formation of a bulk material. In Section 3, we take a look at successful large-scale efforts of ELM engineering, with some commercially successful examples and an overview is provided of biomanufacturing methods and technologies which can be adapted for ELM production, processing, or assisted assembly. 


\section{Section 1. Engineering Cells and Biofilms as Living Materials}

Engineered Components of Biofilm Matrix of ELM-The pillars of the ELM concept are the idea of engineered living cells playing an active role in the tasks of material synthesis, self-organization into higher order structures, and maintenance of those structures in response to environmental stimuli. A natural starting point for these efforts is with bacteria, since they proliferate rapidly and many of them are genetically tractable. Bacteria also secrete and assemble extracellular matrix components, in the form of polysaccharides, proteins, and DNA, during biofilm formation ${ }^{[15]}$. Exopolysaccharides are produced by most species of bacteria as an ECM component. They are structurally diverse and often poorly characterized, encompassing polymers that are linear and branched, and composed of a wide range of carbohydrate monomers. ${ }^{[16]}$ Functionally, polysaccharides serve as a "molecular glue" that allows bacteria to non-specifically adhere to surfaces and to each other, and can also play a role in protecting cells from harsh environments. ${ }^{[17]}$ As an engineering platform, exopolysaccharides are convenient in that they are often the most abundant ECM component in biofilms. However, structural modifications to polysaccharides using genetic engineering are inherently difficult compared to proteins and nucleic acids because of the lack of standardization in biosynthetic pathways across species. Extracellular proteins are also a common bacterial biofilm matrix component in the form of functional amyloids, pili, and flagella. Functional amyloids, in particular, have generated much recent interests as scaffolds for materials engineering. Unlike polysaccharides, protein structure, and to some extent function, can be manipulated with relative ease as the proteins are directly encoded genetically, making them attractive for imbuing ELMs with specific functional characteristics, such as catalysis or specific binding capabilities. Nucleic acids have also been the subject of remarkable advances in terms of structural programming, which could possibly be applied in ELM systems. However, most of this assembly has occurred on the sub-cellular scale and so far, nobody has exploited natural mechanisms of nucleic acid export to induce super-cellular organization, so this section focuses primarily on examples of protein and polysaccharide engineering with relevance to ELMs. To the extent that these biomolecular components, in addition to the cells themselves, are the building blocks for ELMs, controlling their production and programmed organization is integral to the advancement of ELMs. Bacteria secrete a complex assortment of extracellular polymeric substances that include polysaccharides, proteins, and nucleic acids. In this section, we primarily review work that has focused on engineering biofilm polysaccharides and proteins into synthetic materials that are genetically programmable.

Engineering Bacterial Polysaccharides for ELMs-Bacterial cell surface polysaccharides, such as lipopolysaccharides and capsular polysaccharides, are characterized by enormous structural complexity. The structural modification of these polysaccharides brings an opportunity to create a large variety of functional living materials. One example of this is a strategy developed by Yi et al., where fucose, a common bacterial polysaccharide, is modified by using the cell's in vivo biosynthetic systems (Figure 3). This was accomplished by incorporating the GDP-fucose salvage pathway from Bacterioides fragilis into E. coli, replacing the native E. coli GDP-fucose de novo pathway and enabling the integration various non-native fucose analogs. ${ }^{[18]}$ Thus, various chemical functional groups such as azide, alkyne, ketone or amino groups have been metabolically engineered 
into the polysaccharides. Overall, metabolic glycoengineering has been applied much more extensively in the field of chemical biology, where it is used as a labeling strategy after incorporation of azido-sugars into cell-surface glycans. ${ }^{[19]}$ The prospect of using metabolic engineering to create polysaccharide polymers with non-standard chemical compositions is alluring for ELM development. However, issues related to poor incorporation efficiencies of modified sugars may need to be addressed before this strategy becomes more practical for scalable materials fabrication.

Among the diverse exopolysaccharides produced by bacteria, cellulose has garnered the most attention in recent years. Bacterial cellulose has several unique properties -- it is 15 times stronger than plant-based cellulose, biocompatible, and free of hemicellulose and lignin. ${ }^{[20]}$ Due to its properties, bacterial cellulose has a wide variety of existing commercial uses such as high-quality acoustic speakers, medical wound-dressings, and health foods. [21,22] The identification of Gluconacetobacter xylinum (formerly Acetobacter, among other names) as a bacterium whose metabolic byproducts lead to the formation of a thick pellicle at the air-water interface after days-to-weeks of stationary culture was described in the scientific literature as early as $1886 .{ }^{[23]}$ Since then, it has been a topic of much research and even started to be marketed commercially in edible form as "nata de coco" (when the fermentation occurs in coconut water) in the 1960s. More recently, bacterial cellulose has been a point of convergence for materials science and synthetic biology communities, likely for two main reasons: 1) naturally occurring cellulose producing strains like G. xylinum and others are readily available and extremely easy to culture in the laboratory, and 2) in contrast to pellicles made by other bacteria, those made by $G$. xylinum are mechanically robust, selfstanding mats that can be readily subjected to various physical manipulations, including drying to form a "bacterial leather". This interest in bacterial cellulose as a material has fueled new efforts to develop genetic tools to control its mechanical and functional properties, along with top-down fabrication techniques to fashion it into useful morphologies. Indeed, it has become a popular topic of research among a diverse community that spans academics, industry, students participating in the iGEM competition, and even fashion designers. ${ }^{[24,25]}$

Cellulose, like xanthan, dextran, alginate, and other exopolysaccharides, is made through a specific biosynthetic pathway that can be targeted using established metabolic engineering techniques to either enhance biopolymer production or alter its chemical composition (Figure 4a). One straightforward example of this was described recently by Mangayil et al., who increased the production of cellulose four-fold in Komagataeibacter xylinus by recombinantly overexpressing genes from the endogenous bacterial cellulose synthase (bcs) operon (Figure $4 b$ ). Over-expression of all four genes in the bcs operon, which collectively enable polymer biosynthesis, regulation, chain crystallization and export, led to more efficient glucose usage and thicker cellulose films compared to the wild-type strain. ${ }^{[26]}$ However, the mechanical and piezoelectric properties of films made by the engineered $K$. xylinus did not show much appreciable enhancement compared to those created by the wildtype strain, highlighting the need for enhanced hierarchical assembly strategies to complement conventional metabolic engineering if the true potential of ELMs is to be realized. In another example, Florea, et al. report a new cellulose producing strain, Komagataeibacter rhaeticus iGEM, containing a genetically-encoded cellulose production 
circuit. The strain was engineered to be compatible with a "toolkit" for further manipulation, including an annotated chromosome, a small plasmid library, and a set of reporter genes that are all packaged in a way that should make engineering of this strain accessible to a wider audience. They also introduce an inducible system to control cellulose production based on the AHL-based quorum sensing circuit, and demonstrate its utility in spatially and temporally patterning the living cellulose pellicle during the growth phase of the bacteria (Figure 4c). In addition to controlling bacterial cellulose production, this research group was also able to functionalize the cellulose mat by with a range of cellulose-binding domain (CBD) fusion proteins. ${ }^{[25]}$ In addition to these rational engineering approaches to increasing cellulose production, there may be opportunities to employ directed evolution to this challenge. A major hurdle in this effort is the ability to synthesize and characterize materials created from clonal cell populations in high throughput. However, recent advances in droplet microfluidic encapsulation and processing could open the door to screening of large libraries of genetic variants. Indeed, G. xylinus has been encapsulated in a microfluidic droplet system and has been demonstrated to produce cellulose microspheres over the course of several days after encapsulation. ${ }^{[27]}$

Although cellulose is the best studied exopolysaccharide for materials applications, it should be noted that there are others that exhibit complementary properties and are worth further exploration. In one example, Fang J, et al. were interested in curdlan ( $\beta$-1,3-glucan), a biofilm matrix component derived from a commercially available Agrobacterium sp. [28] Although both cellulose and curdlan are biosynthesized using a common precursor (UDPglucose), curdlan is more readily degradable in humans than cellulose due to the $\beta-1,3$ linkage between glucose units, making it potentially valuable as a tissue engineering scaffold. In order to create a material that combined the mechanical robustness of cellulose with the degradability of curdlan, the researchers introduced a single curdlan synthase gene into the cellulose producing strain Gluconobactesr xylinum. Even though curdlan normally has its own dedicated secretion machinery that was absent in the engineered G. xylinum, both curdlan and cellulose were successfully exported to create a composite pellicle with intertwined fibers from both polysaccharides (Figure 4d). The pellicle's surface morphology and hydrophilicity also exhibited properties in between pure Curdlan or pure cellulose materials. Thus, the ability to engineer cell types to biosynthesize and assemble multiple biopolymers simultaneously could represent an interesting entry point into ELMs with composite structures.

The above examples highlight the potential for ELM fabrication using engineered polysaccharides as their structural matrix. Engineered biosynthetic pathways offer new opportunities to control their chemical composition and material properties. On its own, this level of control is valuable from the perspective of biological manufacturing or new polymers. However, it has particular relevance for the development of ELMs, which strive to coordinate control over metabolic processes at the level of individual cells with dynamic self-organization across multiple length scales.

Engineered Protein Structures as Biofilm Matrix Scaffolds-Although proteins are not usually the most abundant component of the biofilm ECM mass, they are attractive building blocks for controlling the assembly of materials with order beyond the size scale of 
a single cell. This is because protein engineering offers control over polymer sequence, which enables programming of sophisticated functions, like specific molecular recognition, ordered self-assembly, and catalysis. Such functions would be difficult to achieve with polysaccharides. Indeed, naturally occurring biological systems employ proteinaceous building blocks like collagens, keratins, and silks exactly for these reasons.

An early success in re-imagining the microbial cell surface as a programmable scaffold for mediating interactions between cells and with other elements of the extracellular environment involved S-layer proteins. ${ }^{[29]}$ S-layers are an ancient cellular encapsulation strategy found in almost all archaea and in many species of Gram-positive and Gramnegative bacteria that relies on the assembly of proteins into two-dimensions (Figure 5a,b). [30] The lattice-like protein monolayers can adopt crystalline patterns with various symmetries, and play a role in membrane-like barrier function, bacterial adhesion and enzyme scaffolding. ${ }^{[31,32] ~[33]}$ By genetically fusing heterologous domains to S-layers, they can become a useful scaffold for creating novel synthetic nanobiomaterials. Indeed, there exists a rich toolset for displaying various domains by fusing them to S-layer proteins derived from Lactobacillus spp. and others, and these have been used for applications that include vaccines, heavy metal bioremediation, sensor diagnostics, and cell-free nanobiomaterials. While the methodology of S-layer engineering and its applications have been reviewed in detail elsewhere ${ }^{[34]}$, here we will highlight a different variant of bacterial cell-surface display that focuses on controlling higher order interactions that might be of particular interest to the development of ELMs. Morais, et al. engineered a consortium of Lactobacillus plantarum to cooperatively assemble an extracellular artificial cellulosedegrading enzyme complex, or cellulosome (Figure 5c). ${ }^{[35]}$ The consortium consisted of three strains - two had been modified to secrete soluble forms of a recombinant cellulose and xylanase, respectively, each fused to a distinct dockerin domain. A third strain utilized a cell wall anchoring scheme to display an assembly scaffold that exploited dockerin-cohesin interactions to bind both enzymes in close proximity to one another. Together, the consortium was able to degrade cellulosic biomass in the form of pre-treated wheat straw with at least as much efficiency as the corresponding soluble enzymes, but with enhanced stability. This strategy of dividing tasks up among different engineered strains may be a promising approach to ELMs that avoids over-taxing the metabolism of any individual cell type.

Another type of biofilm matrix protein that has rapidly attracted interest as a scaffold for materials engineering is functional amyloids. These are secreted by a wide range of bacteria during biofilm formation and assemble extracellularly into nanofibrous meshes that can play structural, adhesive, and biological roles within biofilms. The most well-studied functional amyloid is the curli system, which are produced by $E$. coli and have also been characterized in Salmonella, Citrobacter, and Enterobacter spp. ${ }^{[36]}$ Curli nanofibers from E. coli are formed from the extracellular self-assembly of CsgA, a 13-kDa protein that is secreted as an unstructured monomer, and then polymerizes extracellularly. Several groups have exploited curli fibers as a highly engineerable scaffold for functional materials fabrication. This is usually accomplished by fusing heterologous protein domains with specific functions to CsgA through genetic engineering. Allen, et al. demonstrated that this overall strategy can be used to achieve in situ, extracellular fiber assembly during cell growth. ${ }^{[37]}$ Furthermore, 
by controlling the production of two different CsgA variants using separate inducible systems, they were able to create engineered biofilms composed of curli fibers with gradient or block co-polymer-like nanostructural features. In another example, by controlling the relative concentration of $\mathrm{Csg} A$ and another protein in the curli system, $\mathrm{CsgB}$, along with other aspects of protein structure, like fused linkers, the nanomechanical properties of the curli fibers could be tuned. ${ }^{[38]}$

Several other studies have shown that genetic control can be coupled with microbiallydirected creation of functional materials. In one example, the Joshi group developed Biofilm-Integrated Nanofiber Display (BIND), which initially demonstrated that a wide range of peptide domains could be displayed on curli fibers, and the resulting biofilm materials would be imbued with the functional properties of those domains, for example specific substrate adhesion or inorganic nanoparticle templating (Figure 5d). ${ }^{[39]}$ While some functional properties can be accessed by short peptide domains, other desirable functionalities, like catalysis, required an alternate strategy, since many large domains cannot be secreted successfully after fusion to CsgA. Thus, the authors in a follow-up study employed a popular and highly efficient engineered two-component 'SpyTag/SpyCatcher' covalent bioconjugation technology to immobilize enzymes on the biofilm after they had been fabricated (Figure 5e). ${ }^{[40]}$ In a subsequent report, this approach was extended to include a panel of several covalent and non-covalent conjugation strategies that were mutually orthogonal, enabling multi-enzyme immobilization directly from mixed cell extracts. Thus, the biologically fabricated curli fiber matrix was transformed into a versatile scaffold for multi-step in vitro biocatalysis. Furthermore, because the conjugation domains exhibit different stabilities under different conditions, one enzyme can be removed from a multi-enzyme system without affecting the others. ${ }^{[41]}$ Other recently published papers have focused on the ability to create engineered curli-based biofilms that are electrically conductive. This was first accomplished by displaying conductive gold nanoparticle binding domains on CsgA in order to create a bio-inorganic hybrid material (Figure 5f) ${ }^{[42]}$ Another example involved fusing an aromatic residue-rich domain derived from the PilA protein of the electroactive marine bacterium, Geobacter sulfurreducens to achieve conductive fibers that were completely genetically encoded. ${ }^{[43]}$

In addition to genetic control over nanoscale properties, some progress has also been made toward creating self-standing, macroscopic, living materials using curli fiber engineering. Using an adaptation of a common protocol for amyloid fiber purification, method has been developed to fabricate macroscopic curli-based materials by simply filtering bacterial cultures. This approach works surprisingly well due to two factors: 1) the extreme robustness of the curli fibers, which are resistant to, among other things, proteoloysis, highly alkaline conditions, and heat and organic solvent induced denaturation, and 2) the ability to form aggregate curli fiber mats in cell culture in the absence of $\mathrm{CsgB}$. The resulting filtered biofilms can be used as functionalized filter membranes for selective analyte removal, or can be crosslinked to create sheets of "amyloid paper" that can be removed from the underlying filter paper surface. Because of the robustness of the curli fibers, the filtered biofilms can be used with viable $E$. coli embedded in them, or decellularized to reveal a material from which cells, DNA, and non-specifically bound proteins have been removed. ${ }^{[44]}$ Finally, although these examples of engineered curli fiber-based materials provide a strong foundation for 
ELM development, the living nature of the biofilms has yet to exploited fully beyond the material production phase. In order to realize the true potential of ELMs, as in the tree analogy given in the introduction to this review, the cells embedded in the biofilm material should play a role in continuously remodeling the curli matrix appropriately in response to cues sensed from the environment. As a first step toward this level of dynamism, the Joshi group has recently reported a genetic circuit for curli fiber-based sequestration of mercury ions. A common challenge in bioremediation efforts is balancing the processes of biomass accumulation and metal adsorption in an environment that may be toxic. They report a gene circuit that regulates the production of a mercury sequestration matrix (i.e., curli fibers) with a mercury-responsive promoter (Figure $5 \mathrm{~g}$ ). ${ }^{[45]}$ Thus, the matrix is produced when mercury is present above a critical concentration. Once the mercury has been sequestered and the concentration of free $\mathrm{Hg}^{2+}$ drops below the critical value, matrix production is switched "off", allowing the cell to focus on propagation. A living material with this type of dynamic responsiveness would be ideal for systems that are expected to act autonomously after deployment in the field, since no outside intervention is needed for proper function.

Superficially, curli fiber engineering is about secreting a self-assembling protein from a genetically tractable cellular chassis. This raises a question as to whether this approach could be easily adapted to be compatible with any combination of chassis and selfassembling protein system. Here it should be noted that one of the reasons that curli fibers have proven to be a convenient starting point for ELM design is because the curli system contains several auxiliary gene products that aid in curli biogenesis. For example, $\mathrm{CsgC}$ is a chaperone that inhibits CsgA assembly intracellularly, $\mathrm{CsgG}$ is a dedicated outer membrane transporter, and $\mathrm{CsgB}$ nucleates $\mathrm{Csg} \mathrm{A}$ assembly to form cell-anchored fibers. Thus, researchers attempting to design ELMs based on self-assembling proteins may have to address these challenges before new systems can be successfully designed de novo.

Nevertheless, some progress has been made in reimagining extracellular assembly modes for secreted proteins. In one example, researchers employed the genetically encoded SpyTagSpyCatcher conjugation scheme to create modular extracellular polymers. ${ }^{[46]}$ First, genes were constructed encoding for proteins with form SpyPart-protein-SpyPart, in which arbitrary protein sequences were flanked by different combinations of SpyTag and SpyCatcher. The genes were recombinantly expressed in B. subtilis, an industrially relevant strain known for its prowess in protein secretion. Once the proteins were secreted, they could form longer polymeric structures through spontaneous covalent bond formation. Thus, multiple cell types could cooperate to form completely novel and modular extracellular polymeric structures.

Genetic Control Over Biofilm Formation and Patterning-In order to realize the full potential of ELMs with microbes as the cellular component, extracellular matrix synthesis and assembly must be combined with engineering approaches to control material organization over length scales much larger than individual cells. Here again, the primary inspiration for developing engineering solutions are arrived at by repurposing control mechanisms from existing biological systems. Microbes already exhibit super-structural organization in multicellular communities in the form of biofilms. Biofilm formation is a complex and well-studied process that is influenced by numerous factors, including not only 
matrix production, but also genetic regulation, quorum sensing, cell differentiation, and metabolic changes. These control mechanisms will need to be understood and harnessed in order for ELMs to achieve the type of hierarchical complexity, responsiveness, and longterm viability of biological systems. Significant work has already been completed to apply synthetic biology tools like genetic circuits and engineered environmental sensors to influence processes like biofilm formation, structure, and dispersal. ${ }^{[47]}$

In one of the first examples of rewiring cellular signaling pathways to induce biofilm dispersal, Lee, et al. employed a range of directed evolution approaches to arrive at functionaltering mutants of the SdiA protein, which normally controls quorum sensing in E. coli. ${ }^{[48]}$ Different mutants either promoted or inhibited biofilm formation, primarily by modulating metabolism of indole, the main quorum sensing signal in E. coli (Figure 6a). This demonstrated that genetic rewiring was a viable strategy to control biofilm formation in response to external signals. The same group went on to demonstrate a more sophisticated biofilm dispersal mechanism involving a consortium of two engineered strains of $E$. coli one playing the role of a "primary colonizer" and another playing the role of a "disperser cell". [49] In previous work, the same group had developed two engineered proteins derived from global regulators of biofilm formation processes (Hha13D6 and BcdAE50Q) that were capable of inducing biofilm dispersal. ${ }^{[47,50]}$ The disperser cells were engineered to constitutively produce a Pseudomonas derived quorum sensing molecule, and also BcdAE50Q under the control of an IPTG-inducible promoter. The primary colonizers were engineered to sense the QS molecule and produce Hha13G6 when its concentration reached a critical threshold. Thus, they showed that biofilms could be formed by the primary colonizers inside the microfluidic device, and when the disperser cells were introduced, they would reach quorum, inducing Hha13D6 production and biofilm dispersal in the primary colonizers. Finally, treatment with IPTG would induce BcdAE50Q production in the disperser cells and removal of both cell types from the chamber (Figure 6b). In subsequent work from the same group, a similar strategy was applied to the fabrication of living membranes that had been colonized with "beneficial biofilms" capable of preventing colonization of undesirable bacteria ${ }^{[51]}$.

In addition to controlling the cooperative processes of microbial cells by directly influencing gene circuitry involved in biofilm regulation, other groups have focused more on selforganization of engineered microbial consortia. Tecon, et al. used two strains of the soil bacterium Pseudomonas putida that exhibit mutualistic behavior based on their cooperative metabolism of toluene as a carbon source. ${ }^{[52]}$ They found that by varying factors like initial bacterial concentration and carbon source to influence the nature of the relationship between these two strains (i.e., mutualism vs. competition), they could induce the consortium to form colonies with different patterns on agar plates (Figure 6c). The fact that these strains autonomously adopted these distinct morphologies based on their metabolic dependencies shows promise in terms of future development of pattern forming ELMs with engineered microbial consortia.

Bioremediation is a popular target application for microbial engineering, and ELMs. Indeed, earlier in this review, we highlighted a recent example of a mercury-sequestering engineered biofilm. ${ }^{[45]}$ In another example, Duprey, et al. engineered a strain of $E$. coli to produce a 
"bacterial biofilter" in response to elevated metal ion concentrations. ${ }^{[53]}$ A heterologous metallic transporter, NiCoT from Novosphingobium aromaticivorans was expressed recombinantly in E. coli in order to enhance cellular uptake of nickel and cobalt ions (Figure 6d). This was coupled with a "synthetic adherence operon" which triggered various biofilm formation processes in response to the metals. The resulting strains could sense metals in their environment and produce biofilms that sequestered metals based on cellular uptake.

Ultimately, the field of biofilm engineering is quite large, and in addition to the examples highlighted above, encompasses various approaches and applications, ranging from bioremediation schemes to continuous flow biocatalysis systems ${ }^{[54,55]}$. However, we attempted to focus on examples that are of particular relevance to advancing the concept of ELMs because their aim is to use engineering tools to control spatio-temporal aspects of super-cellular and material organization.

\section{Section 2. Composite ELM Materials}

To engineer materials with additional functionalities, microorganisms can be grown, assembled, mixed or layered in a synergistic way with other materials to create composites. This concept has ample natural precedent in the ability of microorganisms to participate in the biomineralization of inorganic nanomaterials. One example of this would be coral reefs, which are essentially calcium carbonate deposits that are constructed by marine organisms as a shelter. ${ }^{[56]}$ The building blocks for the assembly of these structures are $\mathrm{Ca}^{2+}$ and $\mathrm{CO}^{2-}$ ions that are found abundantly in the organism's environment, but are not produced by the organism itself. Rather, it produces a carbonic anhydrase enzyme that catalyzes the precipitation of calcium carbonate. ${ }^{[57]}$ Although the individual cnidarian organism themselves are millimeter sized, the reefs created by their collective effort can stretch thousands of kilometers, and comprise a dynamic structural support for other marine life that is constantly being degraded and renewed. In a similar manner, other naturally occurring and engineered microorganisms have been investigated for their ability to catalyze the synthesis of organic materials like polymers, or otherwise orchestrate the assembly of non-biological building blocks that can be found in their environment, but that they did not produce themselves. In other examples, pre-fabricated structures composed of synthetic polymers or inorganic materials serve as a scaffold to direct cell growth and behavior. Here engineers can capitalize on the abundance of established micro- and nano-fabrication techniques that can be leveraged to modulate cellular behavior at a range of length scales. However, in order to meet the criteria for ELMs, the cells must behave reciprocally to influence material function or morphology. Indeed, although this emerging field remains somewhat underexplored, there are several notable examples of cell-polymer composites in which the living cells directly orient and align polymers on which they are growing, and even actuate them to produce directional motion. Thus, the living composite materials described in this section exemplify the cooperative programmed action of living cells with externally provided building blocks, pushing the boundaries of performance in biohybrid systems. Furthermore, they can be used for a variety of applications, including sensing, remediation, bio-energy production, microorganism encapsulation, release of functional molecules, and soft robotics. 


\section{Polymer composites: Assembly of functional microorganism-polymer}

composites-Many approaches to biohybrid device design rely traditional materials processing strategies that have been adapted to incorporate living microorganisms and integrate them with synthetic elements. Polymers are some of the most common materials used to create matrices and form materials with living microorganisms. For instance, microorganisms have been incorporated within hydrogels during the polymerization phase ${ }^{[58]}$, or allowed to diffuse into the pores of a pre-existing matrix ${ }^{[59,60]}$. They have also been adsorbed onto surfaces or onto porous supports ${ }^{[61]}$, layered with other materials ${ }^{[62,63]}$, spun, or used as coatings ${ }^{[1]}$. These broad research efforts are now opening new avenues in the field of "smart" and responsive materials design, which can incorporate living elements as essential functional components. In the following section, we explore specific examples of these polymer-cellular composites that could be classified as ELMs as we have defined it here. We also highlight potential applications, the current limitations and future perspectives for fabricating such functional composites.

Swelling and actuation in living composites-A selection of microorganisms can be used to modify the mechanical properties of polymers and also allow them to swell or respond to stimuli. This is the case with Bacillus spores, which are dormant cells encapsulated in a hardy protein-based shell that swell reversibly in response to changes in humidity. Based on the known ability of these spores to undergo swelling-induced change their diameter of up to $12 \%$ in humid versus dry environments, Sahin and coworkers developed a hygro-morphic actuator system. They started by using atomic force microscopy (AFM) to better understand how the hierarchical structure of the spores (made of a core, cortex, inner coat and outer coat) responds to changes in humidity and can act as a high energy density material. The Bacillus spores were then deposited onto polymeric substrates, creating hygroresponsive actuator materials. ${ }^{[64]}$ A dense suspension of spores was deposited as a coating onto a flexible latex rubber sheet. As a function of humidity, the differential strain exerted by the spores resulted in a change in curvature in the latex sheet. In a subsequent paper, the same researchers demonstrate a scaled-up fabrication of an engine driven by water evaporation and propose this as a method to harvest energy from humidity fluctuations that occur near large bodies of water. ${ }^{[65]}$ While the work with Bacillus spores was groundbreaking, the material actuation depends primarily on the biophysical behavior of the spore coat, rather than any active cellular processes. This suggests that even more sophisticated materials could be fabricated that take advantage of the sensing and biosynthetic capabilities of viable cells. Indeed, other research groups have generalized the concept of hygroresponsiveness to include living composites with other microbe-polymer combinations. Notably, Wang, et al. demonstrated that the principle of humidity triggered actuation can be extended to biofilms created with gram positive bacteria, gram negative bacteria, and yeast. Furthermore, they showed that the resulting materials could be used to form wearable devices that are actuated by sweat from the wearer. ${ }^{[66]}$

Microorganism-induced swelling has also been used to fabricate hydrogel materials. Inspired by the production of $\mathrm{CO}_{2}$ gas in food products prepared by yeast fermentation, researchers developed a method to use yeast to induce the formation of pores in cross-linked acrylamide networks. ${ }^{[67]}$ In a "fermentation polymerization" process, Saccharomyces 
cerevisiae cells and sugar were introduced in standard acrylamide polymerization mixture. As the polymerization proceeded, yeast cells simultaneously converted glucose into $\mathrm{CO}_{2}$ and ethanol, and the $\mathrm{CO}_{2}$ bubbles were trapped by the crosslinked hydrogel network, causing the formation of hierarchically ordered pores and gel swelling. The resulting hydrogels with superpores (formed by $\mathrm{CO}_{2}$ bubbles) and macropores (formed by the polymer crosslinks) were shown to have a high absorption capacity. ${ }^{[67]}$

One can now imagine tuning the mechanical properties for several other flexible and soft polymeric materials by introducing responsive spores, microorganisms that can undergo fermentation and release gases or other molecules that could affect swelling or mechanical properties, or cells that can reinforce the structure of composites.

Soft living robots-Although microorganisms are valuable to ELM development as rapidly proliferating, robust, and programmable chassis, eukaryotic cells have also been employed as active components of composite materials. Indeed, when combined with polymeric materials, mammalian cells offer unique opportunities for ELMs with new capabilities, such as coordinated, directional actuation, and even locomotion. In one example of mammalian cells imbuing a material with locomotive capabilities, sperm-flagella inserted in a micro-tube were used to fabricate magnetically-controlled swimmer micro-bio-robots. ${ }^{[68]}$ Cardiomyocyte cells cultured onto polydimethylsiloxane (PDMS) filaments have also been used to produce swimmers. Researchers used the periodic contractions of the cardiomyocytes to generate a bending wave that propagated through the PDMS filament and propelling the swimmer forward. ${ }^{[69]}$

In general, muscle cells have attracted a great deal of interest for the production of responsive machines and soft robots. Parker and coworkers pioneered the fabrication of actuated muscular thin films when they fabricated devices capable of gripping, pumping, walking, and swimming with fine spatial and temporal control. ${ }^{[70]}$ In this work, researchers used the fibronectin-coated micro-contact printed surfaces to seed ventricular cardiomyocytes. Fibronectin-cardiomyocyte interactions guided the inter- and intracellular organization of the cells, which resulted in different actuation patterns for different patterns of fibronectin (Figure 7a). ${ }^{[70]}$ Later, the same group fabricated an engineered soft jellyfish robot that, just like the muscular thin films, relied on the anisotropy of cardiac tissues to produce a robot mimicking a jellyfish. ${ }^{[71]}$ Simulations were used to determine that feeding and propulsion were the key components for jellyfish stroke cycles, and a sheets of muscle cells couples with electrical stimulation was designed to mimic the axisymmetric musculature of the jellyfish (Figure 7b). The resulting 'medusoids' replicated jellyfish swimming strokes. Recently, a stingray-mimicking soft robot guided with optical stimuli was also developed. ${ }^{[3]}$ Rat cardiomyocytes were patterned onto an elastomeric body enclosing a micropatterned gold skeleton (Figure 7c). Optical stimulation allowed for the activation of the muscle cells using optogenetics. The myocytes cells were engineered to respond to optical stimuli via a light-sensitive ion channel [channelrhodopsin-2 (ChR2)]. Researchers then recreated the undulatory motion of a ray by creating a serpentine pattern with the cardiac cells. For maneuvering, the pectoral fins were independently actuated using left and right stimuli at different frequencies. ${ }^{[72]}$ 
Another group has used 3D printing to fabricate miniaturized walking machines referred to as "bio-bots", in which actuation was based on the contraction of cardiomyocytes or skeletal muscle cells. ${ }^{[73] ~[74] ~ W h i l e ~ s k e l e t a l ~ m u s c l e ~ c e l l s ~ d o ~ n o t ~ e x h i b i t ~ s p o n t a n e o u s ~ c o n t r a c t i l i t y, ~}$ their actuation can be precisely controlled via external signaling. Material properties of 3D printed muscle composite were tuned by incorporating extracellular matrix proteins. ${ }^{[74]}$ Apart of from muscle cells, soft robotics concepts could be applied to a variety of cell types and tissues. Making use of 3D printing and other microfabrication techniques, soft robots are now creating a bridge between the fields of living materials and tissue engineering. They also serve as examples of how ELM field can effectively co-opt design principles from more established fields.

Smart living surfaces-"Living surfaces" have also been fabricated with the goal of releasing functional molecules or responding to external stimuli. To do so, living cells can be entrapped in a polymeric matrix that allows them to maintain their viability and produce or consume substances. Initial examples of "living surfaces" were demonstrated by the Stark Lab using fungi to create antibiotic-releasing ${ }^{[62]}$ and self-cleaning ${ }^{[63]}$ surfaces (Figure 8). In these studies, the authors used a three layer structure to create the surface: 1) a base polymeric layer was used as a mechanical support for the living cells, 2) a living layer consisted of the habitat for the living fungi cells, and 3) a porous top layer allowed for diffusion or nutrients and gases required for the living layer, and also acted as a protection against the external environment. ${ }^{[63]}$

To produce antibiotic-releasing surfaces, Penicillium chrysogenum was used as a penicillinproducing fungi strain. The resulting surfaces were shown to sustain a penicillin release above the minimal inhibitory concentrations for penicillin-sensitive organisms for days, and were effective at inhibiting the growth of Gram-positive bacteria. ${ }^{[62]}$ The "living surface" approach differs from traditional materials approaches for releasing molecules in the spatiotemporal production of the functional molecule to release. In traditional materials systems, the molecule is stored inside of polymer layer prior to release, and as it diffuses out, its concentration within the material is depleted. In the "living surface" approach, the functional molecule is produced continuously by the living cells inside of the material. These cells act as continuous reactors consuming nutrients and releasing products in a sustainable manner (Figure 8a).

To produce self-cleaning surfaces, Penicillium roqueforti was used as living layer capable of metabolizing food spills. In the absence of food on the surface of the porous top layer, the living layer was in a dormant state, and could be kept as such for long periods of time. In the event of a food spill, the fungi layer could metabolize the food and grow, thereby creating a self-cleaning surface (Figure 8b). ${ }^{[63]}$ These reports are the first steps toward smart living surfaces that could produce and release, or metabolize and degrade, a molecule of interest in response to external stimuli.

Microbially manufactured polymer matrices-Apart from being able to interact synergistically with synthetic polymeric substances, bacteria are able to synthesize a variety of biopolymers themselves (Figure 9a). Some of these were discussed above with regard to their role as biofilm extracellular matrix components. Indeed, biofilm matrix synthesis, be it 
in the form of proteins, polysaccharides, or nucleic acids, seems to be a widely conserved feature of most naturally occurring bacterial strains. Here we highlight other examples of biopolymer synthesis by bacteria that could be leveraged for the assembly of ELMs. Some intracellularly produced biopolymers are already exploited as bioplastics precursors, and great effort has been undertaken to increase their production through metabolic engineering and optimization of fermentation processes.

Among these biopolymers, polyhydroxyalkanoates (PHAs), a type of polyester, have been widely studied recently, and along with the metabolic pathways involved in their production ${ }^{[75-79]}$. PHAs are attractive because they can be classified as sustainable materials, since they can be produced via bacterial fermentation, are used to produce recyclable and biodegradable plastic products in a scalable manner already (Figure 9b). ${ }^{[75,80]}$ In addition, the biodegradability and biocompatibility of PHAs has made them promising candidates for the fabrication of a variety of medical devices for drug delivery, tissue engineering and other therapeutic applications. ${ }^{[81]}$ Although most current efforts are focused on materials fabrication using isolated and purified PHAs, their properties also make them ideal candidates for ELMs. However, true ELMs created from PHAs would likely require further cellular programming or fabrication techniques in order to promote cooperative extracellular material assembly and modulation.

Bacteria can also be engineered to play an active role in polymerization reactions even without directly leveraging their metabolic pathways, by providing monomers exogenously and relying on the ability of the cells to modulate their own local environment to promote polymerization. ${ }^{[82]}$ This is the case in a recent report where bacteria catalyze atom transfer radical polymerization (ATRP) reactions through their redox metabolism (Figure 9c).

Researchers found that a range of bacterial cell types were able to produce a highly reducing environment near their cell surface that could lead to the formation of $\mathrm{Cu}(\mathrm{I})$ species from $\mathrm{Cu}$ (II) provided in solution, which enabled polymer formation. Positively charged chemical monomers were selected as they could electrostatically bind to the negatively charged surface of bacteria. After polymerization, two fractions of polymers could be recovered: 1) polymers strongly bound to the cells, referred to as templated polymers, and 2) weakly bound polymers, identified as non-templated. Templated polymers exhibited a higher degree of specific recognition for unlabeled cells that were of the same type as the ones that templated them, but not for other cell types. They could then be used, in combination with their encapsulated cell type, to produce larger cell-polymer clusters via self-assembly. Thus, the authors suggest that the "bacterially-instructed" (i.e., templated) polymers could serve as a selective labeling strategy for particular microbial strains. They also demonstrate the compatibility of their system with clickable polymers and postulate that their strategy could be useful for a range of diagnostic applications, for example rapidly isolating and classifying pathogens. ${ }^{[82]}$

Living carbon composites-Living microorganisms have been recently combined with nanomaterials to form bionic composites, or in other words, composite materials that combine advantages of both the living and non-living worlds. When combined, living organisms provide metabolic pathways that can interact with nanomaterials and selforganization properties, while nanomaterials give additional functionalities to the 
composites, such as electronic properties. Several examples of bionic carbon-based composites, involving graphene and nanotube materials, have been recently reported for uses in electrochemical and electronic devices.

Recently, fermentation was used as a method to produce composite living materials with novel and unexpected properties. Inspired by the bread fermentation using Saccharomyces cerevisiae yeast, the Pugno group initially utilized the bubbles formed during yeast fermentation to organize nanomaterials at the water-oil interface of a mixture, and referred to this process as a microorganism nutrition-based strategy to fabricate nanocomposites with intractable polymers. ${ }^{\left[{ }^{[3]}\right.}$ Although the resulting composite materials were porous (due to the yeast-based $\mathrm{CO}_{2}$ formation) and had improved mechanical properties, they did not exhibit any synergistic effects with the surrounding matrix. ${ }^{[83]}$ Nevertheless, they served as a precursor to the development of materials wherein the living cells played a more direct role in the performance of the final composite materials. Follow-on work form the same group described bionic composites of yeast and carbon materials that were produced using fermentation (Figure 10a). ${ }^{[84]}\left[{ }^{[85]}\right.$ First, fermentation was used to produce yeast-CNT bionic composites with improved mechanical, electrical and optical properties compared with yeast and CNTs simply blended together. ${ }^{[85]}$ Through fermentation, Saccharomyces cerevisiae cells were thought to internalize and encapsulate CNTs. In the final composite, CNTs bridged yeast cells. The resulting reduced volume fraction of CNTs within the film made the fermented composite more resistant to fracture, and the CNT bridges between yeast cells contributed to enhancing electron transfer. Second, yeast-graphene bionic composites with synergistic mechanical and electrical properties were produced. ${ }^{[84]}$ In this case, the graphene sheets formed an electrically conductive layer encasing the yeast cells. Again, this composite showed a higher failure strength, and was able to self-repair after placing the composite material containing living yeast cells back into growth medium. With mother and daughter yeast cells forming a "sandwich-like" configuration around a graphene sheet, cracking due to tension along the graphene plane was repaired by reorganizing the graphene sheets between the growing yeast cells. ${ }^{[84]}$ In these bionic materials, both the living cells and the carbon materials contribute synergistically to the final properties of the materials. Lastly, a recent example of a graphene, CNT and liquid rubber composite produced through yeast fermentation was reported. ${ }^{[86]} \mathrm{CO}_{2}$ bubbles produced during fermentation along with collapsed yeast cells resulted in the transformation of conventional silicone rubber composites to auxetic robust rubber. Through these examples, it appears that composite formation through fermentation is a promising approach for the production of light-weight materials with improved mechanical and electrical properties. Furthermore, the cells used in the fermentation can enhance the performance of the materials by inducing self-healing and remodeling the carbon matrix.

Other examples of living composites rely on the self-organization of bacterial biofilms to induce particular morphologies on carbon materials via self-assembly. For instance, a biofilm of Shewanella oneidensis was used to bind and reduce graphene oxide in solution, forming an electroactive thin film (Figure 10b). ${ }^{[87]}$ Synergistic effects were observed between the living cells and the graphene, resulting in improved charge storage and properties. The composite living films were used to fabricate biomemory devices, in which the "write" and "erase" states were controllable through the redox states of the cytochromes 
found at the surface of the $S$. oneidensis cells. ${ }^{[87]}$ This work is an example of living materials where the living cells both serve as templates to coordinate the self-assembly of nanomaterials, change their redox state, and then participate in the function of the final device. The self-assembly of $S$. oenidensis with carbon materials and the use of electroactive biofilms to reduce graphene oxide to graphene were also applied to the fabrication of hybrid microbial fuel cells, ${ }^{[88]}$ and to the synthesis of carbon dots as catalyst for oxygen production. ${ }^{[89]}$

\section{Living cellular composite materials with non-carbon-based matrices-In}

addition to organic polymers and carbon-based nanomaterials, other inorganic components have also been investigated as matrix elements in living cellular composites. Certainly, metallic and ceramic building blocks are commonly used by biology to build load bearing structures like shells and bones. From an efficiency viewpoint this can be seen as advantageous, since ceramic structures can be fabricated by mineralization of ionic species from the environment, which requires less metabolic effort on the part of the cell. Consequently, there is a wealth of existing protein scaffolds, metabolic pathways, and organismal chassis that can promote and control biomineralization processes. These can be altered, enhanced, or recombined in new ways to create ELMs. Accordingly, some current efforts are focused on bio-inspired approaches that hijack cellular processes to biosynthesize hierarchically ordered structures directly, while in other ELMs the bacteria constitute a smaller fraction of the material as a whole, but play a critical role in self-healing and binding processes.

Bio-inspired silica-based biohybrids and ELMs-The natural abundance of silicon and calcium in the environment make them attractive choices as structural building blocks on the part of many organisms. Although examples of mineralized tissues abound, the example of diatoms is particularly relevant for ELMs, since they utilize silicon to create mineralized structures (Figure 11a) that are not only far more intricate than any synthetic counterpart, but also exhibit better mechanical properties. ${ }^{[90]}$ Apart from exoskeleton construction, diatoms also utilize silica for metabolic processes such as chlorophyll synthesis. ${ }^{[91]}$ Some bacteria incorporate silica structures into their anatomy, as in the case of Calothrix thermalis, which forms a silica-based mineral crust to reinforce its cell wall, protect itself from predators, prevent dehydration, ${ }^{[92]}$ and provide resistance to acidic environments. ${ }^{[93]}$ This process is prominent in the hot spring environment where the mineral composition is supersaturated. ${ }^{[94]}$ Plants also absorb silicon in the form of silicic acid $\left(\mathrm{Si}(\mathrm{OH})_{4}\right)_{4}$ and transform it into hydrated and amorphous silica which can be found throughout the plant structure, where it reinforces protective layers and strengthens the cell wall. ${ }^{[95]}$ In animals, silicon plays important roles in the synthesis of glycosaminoglycan and collagen as well as bone formation and is concentrated in the connective tissues of vertebrates. ${ }^{[96]}$ Based on the central role played by silicon in a wide range of biological structures, it is a natural choice for the synthesis of bio-inspired engineered materials. ${ }^{[97]}$

One approach to creating ELMs that incorporate silica involves genetically engineering diatoms directly in order to influence their templating abilities. The first system for genetic transformation of Thalassiosira pseudonana, was described by the Kroger lab in 2006. ${ }^{[98]}$ At 
the time, T. pseudomana was the only diatom to have its entire genome sequenced, facilitating the construction of functional expression plasmids, a constitutive vector and an inducible vector sensitive to a nitrogen source in the medium. The toolbox was initially validated for the expression of recombinant proteins in T. pseudomana with soluble green fluorescence proteins (GFP), but this served as the foundation for engineering of genes involved in the formation of the silica exoskeleton itself, such as the silaffin proteins. The silaffins are known to play a role in the biogenesis of the silica exoskeleton and can be solubilized only when the silica is completely dissolved. Interestingly, protein domains that are genetically fused to the silaffin protein (tpSil3) remain associated with the biosilica and are localized to the cell surfaces of the diatoms (Figure 11b). Moreover, cell-mediated assembly of the engineered biosilica extends the half-life of the surface-displayed proteins by a factor of two compared to proteins immobilized in vitro onto the cell-free silica structure through a silica binding domain. The methodology was used to demonstrate that fusion of enzymes to tpSil3 exhibited enhanced activity compared to soluble enzymes (Figure 11c). ${ }^{[99]}$

With the toolbox established, the group set up a platform for enzyme immobilization that they call "living diatom silica immobilization" (LiDSI). The platform allows for the one-step preparation of catalytic nanomaterials directly from the growth of engineered diatoms, without the need for enzyme purification or chemical modification. By virtue of the nanoporous structure of the diatom exoskeleton, the displayed enzymes exhibit enhanced stability and robust activity. Since T. pseudomana is a photosynthetic organism, the materials can be classified as renewable and environmental-friendly. Importantly, the strategy of genetically fusing enzymes to tpSil3 is also compatible with enzymes that are multimeric, cofactor-dependent and dependent on metal-ions (Figure 11d). ${ }^{[100]}$ They also show that glucose oxidase can serve as a "suicide gene" for negative selections via the production of cytotoxic hydrogen peroxidase. This will be an important tool for the application of directed evolution to nanomaterials synthesis - a design approach that is somewhat unique to ELMs in the materials realm. A further extension of this technology platform includes its exploration as a drug delivery vehicle for anticancer therapeutics. In this variation, the engineered T. pseudomana displays an IgG-binding domain on its surface that enables immobilization of cell-targeting antibodies. The biologically fabricated silica material, together with drug-loaded liposomes incorporated into the nanomaterial structure, form the basis of a chemotherapeutic vehicle that targets and kills neuroblastoma and B-lymphoma cells $^{[101]}$ (Figure 11e). Overall, ELMs incorporating engineered diatoms showcase an unparalleled level of sophistication over nano-scale organization of inorganic structures that can continue to be leveraged for a range of applications.

Bacteria-based self-healing concrete-In contrast to leveraging or enhancing the capabilities of mineralized microorganisms, there has also been progress in engineering mineral deposition using industrially relevant bacterial chassis that do not engage in biomineralization normally. One example of this relates to the development of self-healing concrete. A major frontier in improving the longevity of concrete structures is preventing and repairing crack formation at an early stage. Cracks that form as a result of material defects or as a result of weather induced thermal expansion and contraction can significantly 
compromise the performance and safety of concrete structures, which has costly ramifications for infrastructure maintenance. The Jonkers group, along with others, are developing a solution that relies on the ability of living microbes embedded within concrete structures to fill in microcracks with a mineralized sealant and prevent the cracks from getting larger, thereby extending its longevity ${ }^{[102]}$. To accomplish this, they harness the resilience of spore-forming bacteria in the bacillus genus, including Sporosarcina pasteurii, Bacillus cohnii, Bacillus halodurans, and Bacillus pseudofirmus (Figure 12a). ${ }^{[103]}$ These bacteria naturally secrete enzymes that break down calcium lactate and drive the following reaction that precipitates calcium carbonate to fill in cracks:

$$
\mathrm{Ca}\left(\mathrm{C}_{3} \mathrm{H}_{5} \mathrm{O}_{2}\right)_{2}+7 \mathrm{O}_{2} \rightarrow \mathrm{CaCO}_{3}+5 \mathrm{CO}_{2}+5 \mathrm{H}_{2} \mathrm{O}
$$

However, this process is dependent on the metabolic activity of the microbe, which cannot be sustained within the generally inhospitable concrete microenvironment for the lifetime of the material. Therefore, the concrete is combined with the spores of these alkali resistant bacteria instead. Compared with the bacteria, the spores are incredibly resistant to a wide range of harsh conditions, but can germinate when water infiltrates microcracks in the concrete. The revived bacteria then drive the calcium carbonate precipitation using small amounts of nutrients embedded in the concrete to fill cracks. ${ }^{[102]}$

Subsequent work has focused on improving the performance of the self-healing concrete through a combination of bacterial encapsulation strategies and optimization of the concrete fabrication protocols. The reported longevity of the self-healing effect for first generation versions of the bacteria-loaded concrete was on the order of one to two months, which was significantly shorter than the inherent viability of Bacillus spores under more amenable conditions. ${ }^{[104]}$ This was remedied first by encapsulating the spores in a porous clay scaffold, which protected them from compression-induced death during the concrete drying process. This restored much of their inherent viability and extended the crack healing longevity to more than 6 months (Figure 12b). However, in order to achieve the desired crack healing performance, the clay scaffold had to compose such a large volume fraction of the final concrete structure that its mechanical performance suffered. ${ }^{[102]}$ Other research groups have employed other materials to protect the bacteria for the use in self-healing concrete. For example, Wang et al. demonstrated pre-encapsulation of Bacillus sphaericus spores in silica gel and polyurethane before incorporation into the concrete (Figure 12c), with the former scaffold resulting in higher bacterial activity, but the latter resulting in better mechanical and water permeability outcomes for the concrete after induced cracking. [105] Follow-on work has demonstrated that the bacteria and calcium lactate can be encapsulated in metakaolin and aluminosilicate "geopolymers" before incorporation into the concrete to minimize decreases in mechanical properties that result from large volume fractions of weaker encapsulation materials like organic polymers and silica. ${ }^{[106]}$

Biologically fabricated bricks-Finally, a similar concept of bacterially-produced calcium carbonate as a binding material has been applied to the fabrication of bricks for use in building construction. Brick-based masonry remains a prevalent paradigm for building construction in much of the world, but conventional brick manufacturing also has 
detrimental environmental consequences. Although the raw materials for brick construction (i.e., earth) are abundant, the kiln-based firing process that is used to create long-lasting, durable bricks is a major contributor to non-renewable energy usage and environmentally harmful emissions ${ }^{[107]}$. Thus, microbially-induced calcite precipitation (MICP) is being investigated as an alternative approach to enhancing the structural performance of bricks. This scheme primarily makes use of the ability of certain microbes to secrete the urease enzyme, which generates carbon dioxide from urea and catalyzes the formation of calcium carbonate in the form of calcite. When this process, which takes days-weeks, is performed in a brick-shaped mold, the calcite serves as a matrix that binds clay or sand particles together to form a brick. Although there are few literature reports that quantitatively compare the structural performance of bio-calcified bricks to that of conventional fired clay bricks, one paper reports compressive strength values of $\sim 8 \mathrm{MPa}$ for bricks cured in an inoculum of Bacillus pasteurii for 28 days, which is 2-3 fold lower than conventional fired bricks ${ }^{[108]}$. The concept of "farmed" bricks is the basis for a start-up company (bioMASON), which has garnered much interest in the approach (Figure 12d). The company claims that with genetic engineering they can create glow-in-the-dark bricks, pollutant-absorbing bricks or waterdetecting bricks. ${ }^{[109]}$ The ultimate goal for these living calcium-carbonate producing biomaterials, such as self-healing concrete and MICP bricks, is to reduce the cost of structural maintenance, protect structural integrity and decrease pollutant production from the brick manufacturing process.

Multi-organism living composites-Lastly, the simplest approaches to creating ELMs rely on a single cell type that is programmed to produce, secrete, and assemble biopolymeric building blocks. However, engineering a single cell type to perform all the necessary functions can be complicated and inefficient. Even in most naturally occurring microbes, for example, biofilm matrix production is a highly regulated process. Matrix production is metabolically taxing and must be balanced with growth and long-term viability of the colony. Thus, when thinking about engineered systems, an alternative strategy could be to divide metabolic tasks up between two different cell types or even species in order to simplify the production of the material as a whole. Taking it a step further, one could even think about making composite living materials in which two or more cell types enact complementary or synergistic functions. While the growth of different microorganisms and their symbiosis while co-cultured have been studied extensively by ecologists and biologists, few composite materials have been fabricated using two or more microorganisms with complementary or mutualistic functions.

One inspirational example of two microorganisms that can grow into a three-dimensional soft material is the case of the bacteria Acetobacter aceti and the photosynthetic microalgae Chlamydomonas reinhardtii. Like many other Acetobacter species, A. aceti normally produces cellulose mats at the air water interface when grown in liquid cultures, due to its need to remain close to an oxygen source, and produces acetate as a byproduct. In contrast, C. reinhardtii thrive in an acetate-rich medium and produce oxygen as a byproduct. By growing these two microorganisms together, researchers were able to create composite materials with cellulose produced by Acetobacter acting as a matrix that favors the growth and entrapment of microalgae cells (Figure 13). In return, microalgae produce oxygen that 
can be used by the bacteria and allows them to form cellulose uniformly within the culture medium, as opposed to only at the water-air interface. As a result of this mutualistic relationship, a soft biomaterial was formed in situ. The cells embedded in the material could remain alive for up to 7 days, and the material, despite having a lower mechanical strength than bacterial cellulose produced from monoculture, was strong enough stably adopt the shape of the container in which it was grown. Thus, the researchers propose this as a method to create living materials that can be simultaneously grown and molded into a variety of selfstanding three-dimensional shapes. ${ }^{[110]}$

With the increasing interest in understanding the systems biology and metabolic dynamics of microbial consortia in various environments, the groundwork for future mutli-organism living materials fabrication efforts is currently being laid. Indeed, a great variety of microorganisms exist that can produce different types of extracellular polymeric materials, and mutualistic growth strategies could be expanded to a wide array of multi-organism composites. Living microbes could be further engineered to interact with one another to produce materials with novel properties and improved mechanical properties.

\section{Section 3. Large-scale ELM Fabrication / Manufacturing Methods}

Much of this review thus far has focused on small-scale materials engineered in laboratoryscale experiments. In this section, we will look at the efforts for the engineering of largescale multicellular tissues and also explore the challenges of scaling up living materials production and inspiring glimpses into their tangible implementation. For applications beyond that of specialized markets, true integration of ELMs into everyday life will require scalable fabrication paradigms, both in terms of making larger material structures and in terms of making larger amounts of material. This may require new infrastructure investments in order to compete with material economies with which we are more familiar, like plastics, ceramics, and electronics. However, in the nearer term, there are many challenges that must be faced to translate the innovations from the previous sections of this review into ELMs that can be applied in a practical manner. One frontier involves making ELMs that are true tissues, in which the cells primarily exist in a multicellular state, generating and maintaining ECM dynamically over long periods of time. In contrast to bacterial biofilms, the cells that might be used here would not naturally have a planktonic state, and the resulting ELMs would thus have higher structural stability during assembly and maintenance. Also, these tissues will need to have more sophisticated architectures that parallel what biological materials have evolved for nutrient and waste circulation in order to allow for stable growth and maintenance of much larger structures. In this section, we review current successful materials platforms categorized as ELMs, some of which are commercially successful, that are used to generate large-scale materials. Throughout this review, we consider an apt description of large-scale materials to be that of sufficient size for manipulation by hand. We then explore various traditional manufacturing methods that are suitable for the scalable production, isolation, and processing of biomass such as biofilms, due to the amount of earlier focus on biofilm-based systems. Finally, we examine the use of several top-down assembly methods for large-scale ELM fabrication and the potential for synthetic morphogenesis efforts in designing true bottom-up autonomous ELM assembly. 
Tree Shaping-An obvious example of unengineered tissue materials we use every day on such a scale is wood. From paper airplanes to Stradivarius violins to hefty crossbeams, wood is a once-living material which, quite literally, is a foundation on which much of our civilization is built. Structures have also been constructed which use living trees, known as 'tree shaping,' in which living woody plants are continually forced by manual manipulation and grafting to attain functional or artistic forms (Figure 14). Sculptures, tables, chairs, and living spaces have been created using tree shaping. In India, the aerial roots of Banyan trees have been manipulated to create strong living bridges capable of holding up to 50 people. Although tree shaping utilizes physical manipulation instead of genetic manipulation to control one particular aspect of the material (structural form) we can consider it an excellent example of how humans have harnessed living tissues to create macrostructures. Efforts to bring tree shaping into more sophisticated engineering spheres include a concept design for a modern eco-friendly home based on tree-shaping using computer numerically-controlled (CNC) scaffold design ${ }^{[111]}$. Another parallel effort to integrating such tree shaping into modern building technologies has been explored as an architectural engineering approach known as Baubotanik ${ }^{[112,113]}$, developed at the University of Stuttgart. Another exciting area in this area is the genetic manipulation of trees to alter the properties of the resulting wood, such as lignin content, growth rate, or pest resistance. However, progress in these efforts is tempered by significant concerns regarding the environmental impact on their release ${ }^{[114,115]}$.

Mycelial Materials-One of the most commercially successful large-scale living materials is fungal mycelium that can be grown from agricultural waste and molded into various shapes (Figure 15). These 'mycelium materials' are largely based on white- and brown-rot fungi that are capable of digesting lignin and cellulose. Commercialized by Ecovative Design with an initial focus on environmentally-friendly packaging and building materials, this technology is claimed to be capable of modulating the material properties of the mycelial structures by altering the mixture of fungal species ${ }^{[55]}$. A different company, Mycoworks, employs an analogous process using mycelium to generate leather-like materials and even bricks for home building ${ }^{[84]} \cdot{ }^{[84]}\left[{ }^{84]}\right.$. Given the rapid growth of mycelia in comparison to other multicellular tissues, the ease of controlling the final form through the use of molds, and the potential of altering the physicochemical properties of the hyphae, this technology has great promise for the wide-scale implementation for engineered living materials. Hyphae biotemplating would greatly expand the spectrum of uses for mycelial materials by combining the rapid growth potential of fungi with the desirable material properties of inorganics, as demonstrated in several studies ${ }^{[116,117]}$. A recent publication has also characterized the physical properties of the mycelial materials and potential routes of altering these properties by simply adjusting the feedstock ${ }^{[118]}$. Aside from structural materials, this technology also has potential uses in advanced applications such as biologically integrated electronic circuits, as shown in a study that uses integrated circuits to direct the growth of fungal hyphae ${ }^{[119]}$. One fertile area for expanding the functional parameters of mycelium materials is through engineering the extracellular polymeric substances (EPS) generated by the fungi. However, currently little is known of composition and biosynthesis of EPS from these lignin-digesting fungi, other than the fact that it is dominated by polysaccharide networks that serve as immobilization sites for secreted 
enzymes or as structural sheaths that surround the hyphae ${ }^{[120,121]}$. With recent advances in CRISPR-Cas9 tools for genetic manipulation of filamentous fungi ${ }^{[122]}$, the prospect of genetically tailored mycelium materials is within reach.

Bacterial Cellulose-Bacterial cellulose obtained from Acetobacter xylinum and similar cellulose-generating bacteria described in Section 1 can also be considered a successful example of scalable implementation of a living material, although like the examples above, engineering on larger size scales primarily consists of physical manipulation to control the functional morphology of the natural material. For example, bacterial cellulose materials have been used commercially in a number of specialty applications (Figure 16). Its strong mechanical properties have been used to create high-strength paper and unique acoustic properties have been led to their use as diaphragm membranes for high-end audio speakers ${ }^{[123,124]}$. Bacterial cellulose is being explored for electroactive paper for advanced displays $^{[125]}$ and energy storage devices ${ }^{[126]}$. The use of these bacterial-cellulose pellicles as textiles has been explored through concept studies using the cellulose as the primary structural material in garments ${ }^{[77]}$ as well as environmentally-friendly architectural materials $^{[48,127]}$. Current commercialization efforts include a number of biomedical applications, including burn dressings and tissue engineering scaffolds, spearheaded by various companies focused on bacterial cellulose materials ${ }^{[49-51]}$. Looking beyond highmargin biomedical applications, a major barrier for widespread commercial adoption of this versatile material is high production costs, due to the inefficient culturing process of the bacteria - pellicle formation on the benchtop can take a week or longer. Ongoing advances in understanding the cellulose synthesis apparatus in these bacteria, engineering synthetic biological control of bacterial cellulose production, and the exploration of other microbial chassis, such as photosynthetic cyanobacteria, will be critical in improving the production yields and lower culturing costs ${ }^{[128,129]}$.

Scalable Traditional Production Methods for ELMs-In contrast to biomaterials created from purified biopolymers, the concept of ELMs is that the self-growing material is assembled or fabricated close to its final form, with no significant processing required. However, one significant challenge is the choice of production methods, which can range from small batch analytical scales to more substantial semi-preparative scale to full-on industrial-level scales. The vast majority of academic studies for biologically-derived materials focuses on protocols for analytical scales, and will not be discussed in this section. Of particular interest for the large-scale utilization of ELMs are the semi-preparatory and industrial level fabrication methods. The process can be divided into two stages, first is the production of the biomaterial itself, usually through in situ biosynthesis, and second is the processing of the material to obtain a desired level of purity and/or form. An alternative method is also reviewed in which 3D printing allows for cells and feedstock to be spatially arranged in a pre-determined design format.

For ELM materials composed of bacterial ECM such as functional amyloids or cellulose, pertinent bulk production methods include traditional bioreactors, fixed/moving-bed bioreactors, hollow-fiber bioreactors and static bioreactors (Figure 17a). Although there a multitude of designs for specific applications, these are the basic bioreactor production 
platforms that have been employed for large-scale biomaterials (e.g., biofilm) production. Biomass produced this way has most commonly been used for biotransformation reactions. The simplest bioreactor design is a static bioreactor, in which the culture is left undisturbed. Static reactors are relatively cheap to build and are ideal for the production of materials that are assembled in situ as floating pellicles, such as bacterial cellulose. However, due to the lack of efficient mixing for nutrient and waste transfer, the cultures are often slow-growing and thus throughput is a concern. Attempts to increase mass transport in these reactors using microbubbles to stimulate convection for mixing, known as an Airlift Reactor, has been shown to significantly increase the production yields of bacterial cellulose ${ }^{[130,131]}$.

One of the most common bioreactor designs in bioprocessing is the Continuous Stirred Tank Reactor (CSTR), which employs an impeller to continually stir the culture while nutrient feedstock is added at the same rate as the product is removed. Such traditional CSTR bioreactors would be optimal for ELM materials in which a high amount of secreted selfassembling biomaterials can be formed from cells in a planktonic state. However, if the desired materials assemble more efficiently at an interface, as is common with biofilms, CSTRs would not be suitable, as they are designed for high biomass production of planktonic cultures and the high shear forces from the impeller may (depending on species) result in poor biofilm formation and biomass retention ${ }^{[132,133]}$.

For such interfacially-assembled ELM materials, a critical factor in scalable production becomes increasing the available adsorptive surface area to maximize cellular or ECM attachment in the biofilm. This is typically done by supporting structures that serve as highsurface area growth substrates, provide a viable microenvironment for biofilm growth, and offer some protection from shear forces during mixing. The choice of supports is varied in composition and geometries, and includes glass beads ${ }^{[134]}$, glass wool ${ }^{[135]}$, carbon fiber ${ }^{[136]}$, bonechar ${ }^{[137]}$, porous brick particles ${ }^{[138]}$, polymeric supports ${ }^{[139]}$, wood chips ${ }^{[140]}$, and metal structures ${ }^{[141]}$. Reactors utilizing solid supports are very common in chemical processing and these strategies have been adapted for interfacial growth of biofilms as well. These biofilm bioreactors can be categorized as fixed-bed or moving-bed bioreactors (FBBR or MBBR), based on whether the solid supports (bedding) are immobile or freely floating, respectively. In both cases, the biofilms grow on the surface of the supports while a variety of reactor architectures enable introduction of the necessary nutrients, gases, or substrates (feedstock) to the cells and removal of wastes and products. Two of the major designs of FBBRs are Packed Bed Reactors (PBRs) and Trickle-Bed Reactors (TBRs). In PBRs, feedstock is pushed up from the bottom of the reactor, percolating through the solid support bed structures, and products are obtained from the top of the reactor. TBRs have the reverse process, as feedstock is introduced at the top, allowed to trickle down by gravity, and any products are collected at the bottom of the reactor. Both PBRs and TBRs have been used for the successful production of biofilms for biocatalysis applications, but typically not for materials production. PBRs have primarily been used in the field of tissue engineering, having directly borrowed much of the PBR design from synthetic chemistry and applying it to bio-artificial tissue development ${ }^{[142]}$. TBRs have been employed in wastewater management systems for the bioconversion of contaminants using natural biofilms ${ }^{[143,144]}$. 
A number of specialized bioreactor designs exist for the growth of interfacial cultures. The most popular of these bioreactors, known as Moving-Bed Bioreactors (MBBRs) are used extensively at industrial scales by water treatment and decontamination facilities, in which naturally occurring biofilms are used to purify waste or contaminated water. These bioreactors were designed to maximize biofilm growth under low nutrient, low resource, and low footprint conditions but yet maintain a wide range of wastewater flow-through per day ${ }^{[145]}$. MBBRs are a variant of the traditional PBRs. A key part of MBBRs is the solid support material known as the 'biocarrier', often made of inexpensive polymer structures that provide a high-surface area attachment sites for the cells of interest. Rather than the bed material being relatively immobile, as in PBRs, in MBBRs these biocarriers are able to move throughout the reactor, maximizing contact with nutrients and/or the contaminant substrates to be bioconverted.

Most of the bioreactor designs here were developed for bioconversion applications rather than harvesting of the biomass as a material. As current synthetic biological reactor development reaches pilot level production in industry, it is expected that the designs described here will be optimized for biomass production and recovery, similar to how the fundamental chemical reactors were repurposed for biological systems. Potential bottlenecks for the large-scale bioproduction of engineered living materials are the harvesting methods for removal of the materials from any bedding materials. There is great potential for using bedding materials that will be integrated in to the final material so that no separation is required, or using bedding/biocarrier materials that are eventually degraded or converted by the engineered cells.

Post-production Processing - Purification and Dewetting-After production in a bioreactor, one significant step is the removal of the biomass from the culture media. For most current biotechnological applications, the bioconverted media is the end-product (or contains the desired product). For ELM production, this process is inverted -- the biomass material will be the desired product. Within this process, steps can be added to wash away undesired impurities from the material to attain a desired purity level. Filtration is one of the simplest methods that scales well from analytical to preparatory levels. In the laboratory, vacuum-assisted filtration in combination with denaturant chemicals can be used to isolate the desired ECM from other cellular material, allowing for post-biosynthesis purification of these materials. Recently, a method has been developed for the vacuum-assisted filtration of preparatory-scale isolation of highly-pure engineered curli nanofibers ${ }^{[4]}$ (Figure 17b), which can then be used in downstream fabrication methods, such as spin casting into thinfilms. A similar strategy that is used widely at industrial scales to remove biomass from filtrate is press-assisted filtration, where the pressure required for the filtration process is provided by a series of roller pins that compresses the crude sample which is sandwiched between two filter membranes (Figure 17c). This highly efficient strategy is used throughout the paper and water purification industries for the removal of solids from liquids. One key aspect of the post-production process is establishing the acceptable limit of purification for an ELM, which will need to be empirically determined for each material and application. On one hand, cells could play important roles in the continual maintenance and adaptability of ELMs, which could be important for sensing and actuation. However, cellular by-products or 
the cells themselves could also compromise the safety, long-term stability of the material, or the reproducibility of the manufacturing process. Will the cells need to be rendered nonviable for safety concerns? Will the cells need to be completely removed from the engineered material in order to attain the desired mechanical or physicochemical properties? These questions will likely need to be answered on a case-by-case basis for each application.

In situ ELM Production-While the above sections attempt to frame prospects for largescale ELM manufacturing within the framework of current bioproduction and bioprocessing methods, there are exciting possibilities for in situ generation of biomaterials using ELMs. The ultimate goal in such a scenario would be for the acquisition of the necessary energy sources, metabolites, and basic building blocks for organismal replication and materials production across multiple size scales to all occur autonomously and without the need for a reactor vessel. As in the case of a seed growing into a tree, would only occur given the appropriate conditions that foster cell growth and hierarchical self-assembly. Of all current examples, the mycelium material is the closest to in situ production without a traditional bioreactor, although it still requires multiple user-mediated steps and a structural mold to convert feedstock into a desired object. It is likely that significant advances in synthetic morphogenesis and biosynthetic materials production would need to occur before a paradigm for truly autonomous ELM fabrication could be implemented, especially for ELMs with complex hierarchical structure and functional performance features that rival conventional materials. Challenges in synthetic morphogenesis to enable bottom-up hierarchical assembly through purely biological programming are reviewed in a separate section further below. However, two currently existing technologies allow for top-down manufacturing of ELMs with controlled spatial positioning, which we take a look at next: electrospun materials containing living cells and 3D printing methods.

Electrospinning of ELMs-Electrospinning has been used to produce fibrous materials containing living cells that, supported with a polymeric matrix, can accomplish a function. By extension, electrospinning techniques developed for biohybrid materials could be extended to ELMs. Figure 18 shows examples of yeast and bacterial cells encapsulated in core-shell electrospun fibers. ${ }^{[146]}{ }^{[147]}$ In these examples, living cells were encapsulated in a water-soluble biocompatible polymeric core that provides a favorable environment to maintain cell viability. Simultaneously during electrospinning, a second needle can be used to form a shell around the biocompatible core fiber. ${ }^{[146]}$ A shell layer can alternatively be synthetized via chemical deposition after the spinning of the core. ${ }^{[147]}$ This shell layer provides the structure its robustness and durability by preventing dissolution of the watersoluble core. In these electrospun fibers, yeast cells were demonstrated to remain functional and capable of biodegrading polyphenols and producing ethanol (Figure 18a). ${ }^{[146]}$ Composite fibers containing bacterial cells Micrococcus luteus and Nitrobacter winogradskyi were used to sequester gold and remove nitrate from solutions, respectively. [147] The bacteria-containing fibers were mounted onto a wire frame to create a mesh (Figure 18b) that could conveniently be used to perform metal sequestration. The meshes could then be cultivated, and living bacteria could be recovered and re-grown from the fibers. ${ }^{[147]}$ Apart from these core-shell examples, electrospinning has been used to encapsulate bacterial cells for other purposes such as drug delivery ${ }^{[148]}$, and to entrap living 
cells within the pores of a matrix of fibers. ${ }^{[60]}$ The strategy of direct cell encapsulation within fibers with a robust shell material could be further developed to entrap or thread engineered microorganisms and their extracellular matrices. Electrospinning is a promising approach to fabricate large scale materials like meshes and fabrics, and to create living wearable devices and clothing.

3D printing of ELMs-3D-printing technologies have garnered much excitement in the past decade as a manufacturing platform that has made significant inroads for prototyping, architectural construction, and bioprinting. The process of bioprinting uses biologically active cells, often contained in some hydrogel matrix that preserves viability, and precisely deposits them with micrometer resolution to create three-dimensional living composites. The precision of this process is facilitated by computer numerically-controlled (CNC) robotics or photolithography. Several different cell types can be integrated into the structure simultaneously as distinct "bioinks," to create complex three-dimensional objects. This spatially programmed approach allows for the development of anisotropic ELMs with arbitrary shapes, in contrast to current demonstrated efforts of monocultures or co-cultures that lead to homogeneous materials or poorly ordered composites, respectively. In addition, programmed quorum-sensing circuits in these spatially arranged cellular materials can be used to create dynamic ELMs that change in space and time. Bacteria of different species have been encapsulated in photo-crosslinked 3D gelatin structures as small as a few microns by the Shear group ${ }^{[149]}$ (Figure 19a,b). These structures were designed as small microscopic chambers to investigate the dynamics of diffusible effectors between polymicrobial communities. Structures containing multiple bacterial chambers with interconnecting "hallways" as well as nested chambers containing distinct bacterial species were demonstrated using this technique. The smallest fabricated chamber contained a volume of only 1 picoliter. Although these 3D-printed chambers have only thus far been utilized as analytical tools, they could potentially be adapted for ordering specific ELM-producing cells in voxels to create well defined anisotropic materials. A lower cost alternative has recently been demonstrated that uses a modified inexpensive consumer 3D printer (Figure 19c). By integrating a computer-controlled syringe pump with the $3 \mathrm{D}$ printer, the authors were able to print genetically engineered E. coli embedded in an alginate bioink ${ }^{[150]}$. Although the 3D resolution of the structures was on the order of 1-2 millimeters, given the inexpensive build and low resource requirements, this bacterial bioprinter system should enable the broader exploration of 3D-printed ELMs. Currently, the vast majority of funding and effort for 3D bioprinting is focused on the development of ex vivo synthetic tissue and organ engineering. Although this review is primarily focused on ELMs broadly, we recognize that tissue and organ engineering efforts are closely aligned with ELM technology, as organs are assemblages of cells that create an ordered hierarchical structure composed of themselves and the ECM that they generate to perform a physiological function. Thus, many of the current efforts to use 3D printing to assemble complex organs by spatially seeding cells in a biodegradable matrix, such as the recent generation of an artificial kidney structure by Homan and colleagues ${ }^{[151]}$ (Figure 19d), hold much potential for adaption for the fabrication of complex ELMs as well. 
Synthetic Morphogenesis: Enabling Complex Self-assembling ELMs-Much of this review has focused on examples which have explored the most basic embodiment of ELMs, that is, the coupling of living cellular systems with in situ materials production. Functionality can be programmed in at the genetic level and most of these systems utilize the principles of self-assembly either as part of the extracellular materials production (e.g., programmed curli nanofibers) or for the formation of higher-ordered structures with unique properties. A significant remaining challenge is developing the ability to precisely engineer complex structures that are hierarchically organized seamlessly from the nanoscale to macroscale, as living organisms are capable of fabricating. One powerful aspect of structures created by living systems that is yet to be fully recapitulated as an engineering principle is the ability to harness self-assembly across these vastly different scales to create emergent properties where the sum is more than its constituent parts. While current attempts to emulate such complexity borrow from top-down approaches such as the 3D printing technologies described above, a true ELM that is capable of achieving the structural and functional complexity of living organisms will require genetically encoding the dynamic growth, hierarchical organization, and temporal modulation of such biological structures. The fields of evolution and developmental biology have endeavored to elucidate such principles that underlie the spatial and functional organization of tissues and organisms. In much the same way that synthetic biology has attempted to derive engineering principles from genetic and biochemical principles of systems biology, a new effort at this time of this review's publication is attempting also integrate developmental biology principles for the artificial design, programming, growth, and maintenance of complex biological structures. This nascent sub-discipline of synthetic biology is known as 'synthetic morphogenesis' and is covered in detail in an excellent recent review ${ }^{[152]}$.

In the field of biological development, the process of creating higher-order structures from genetically encoded processes is known as molecular morphogenesis, in which overlapping tissue-level modular programs enable structural and functional changes through the induction of cell growth, alteration of gene expression, and/or programmed cell death. These morphogenetic modules exert their control over large cell populations through recurrent motifs such as spatial cellular signaling gradients, intercellular feedback loops, and mechanical stress transmitted through the $\mathrm{ECM}^{[153-158]}$. The integration of these various developmental programs results in large-scale pattern formation and defined multicellular structures arising from collective programs occurring on a local and global scale. The majority of current synthetic morphogenesis efforts are focused on biomedical applications, particularly tissue and organ engineering for the replacement of diseased and/or damaged tissues. Thus, much of morphogenic engineering is focused on the design of biomaterials that exhibit tissue-mimicking qualities related to their mechanical properties and/or biochemical functionalization. These material properties then provide cues to the cells in a spatially defined manner to induce cellular differentiation, large-scale tissue reorganization, and organ-level functions, sometimes using methods such as 3D bioprinting as described above.

Of particular import to our focus is the potential for using synthetic morphogenesis approaches to design and modulate ELMs of a degree beyond what has been heretofore 
accomplished. This new generation of ELMs will allow hierarchical control and programmed anisotropy at various scales, vastly increasing the complexity and functions of materials that can be engineered. As a comprehensive systems-level understanding of multicellular biological structure formation is currently lacking, most of the early research of interest has focused on attempts to model and engineer simple patterns using synthetic biology circuits using intercellular communication modules. The recurrent biochemical strategies that Nature uses for morphogenesis can be classified broadly into four motifs ${ }^{[159]}$ : the 'French Flag' model, phase separation, rule-based mechanisms, and Turing pattern models. The first of these, the 'French Flag' model ${ }^{[160]}$, generates positional information in a tissue through the use of a diffusing signal, known as a morphogen. Morphological differentiation of the cell population (analogous to the different solid bands of the French flag) occurs as a result of threshold-based cellular responses to the morphogen gradient. One of the earliest examples of synthetic biological patterning by Basu, et al. employed acylhomoserine lactone (AHL) based bacterial quorum-sensing modules to create a banddetector circuit that is able to detect a specific concentration range of AHL molecules [161] (Figure 20a, left). In the presence of 'sender cells' that generate an AHL gradient, cells harboring the band detection circuit are activated only when a specific concentration of AHL is encountered, resulting in spatially defined gene expression. Bulls-eye patterned differential populations of bacterial cells were created, as well as more complex shapes based on the initial positioning of the sender cells (Figure 20a, right). A subsequent effort integrated this AHL-based band detection system with an engineered light-responsive genetic switch to create an 'edge-detection' circuit where only cells on the periphery of a light-dark zone are activated ${ }^{[162]}$ (Figure 20b). This approach enables the versatile creation of edge patterns of various shapes through the use of photomasking techniques. A further study by Liu and others integrated the AHL-generating circuit with bacterial motility to create an expanding population of bacterial cells that self-organized into tunable periodic stripe patterns ${ }^{[163]}$ (Figure 20c). Another developmental patterning motif is phaseseparation, in which cells self-organize based on their propensity to associate or adhere, analogous to oil-water interfacial dynamics ${ }^{[164]}$. Also referred to as differential adhesion, different cell types coalesce and separate to form diffuse granular patterns. Artificial recapitulation of this morphogenic strategy was recently accomplished for the first time using mammalian cells engineered to express two cadherin heterotypes to generate complex 2- and 3-dimensional patterns ${ }^{[165]}$ (Figure 20d). Aside from the examples above, the remaining two classes of morphogenic motifs, rule-based mechanisms and Turing patterns, have yet to be engineered in a synthetic system. Rule-based mechanisms encompass those in which simple rules, such as cell morphology, growth dynamics, or death, lead to large-scale self-generated patterns. Examples include fractal formation of bacterial populations ${ }^{[166-168]}$ (Figure 20e), branching of colonies ${ }^{[169,170]}$, and wrinkling architectures in biofilms ${ }^{[171]}$. Turing patterns (TPs), also known as reaction-diffusion models, were proposed by Alan Turing in $1953^{[172]}$ and are characterized by feedback interactions between diffusing morphogens. These patterns are not wholly dependent on the pre-pattern state (i.e., the local position of the 'sender' cells), allowing for self-regulating patterns. They are thought to underpin a wide spectrum of developmental processes, from tissue patterning in animals (e.g., zebra stripes) to bilateral symmetry in animals to limb development. Although the development of synthetic circuits for TP formation would be a powerful tool in the synthetic 
morphogenesis toolkit, to date there has been no demonstrated example. Current obstacles include the required precision of the design space for TP dynamics, as well as the lack of synthetic biological parts that fit the required diffusion and feedback parameters ${ }^{[159]}$. As these emerging synthetic morphogenesis efforts begin to establish robust engineering principles for multicellular patterning and coupling of functional structures across hierarchical scales, we anticipate a synergistic integration with biofabricated programmable matter, leading to a new generation of ELMs.

\section{Conclusions}

This current manuscript reviews key advances in the development of ELMs, advanced materials that utilize biological cells to fabricate, assemble, and maintain the material. The cells act as living nanomaterials factories and thus the ELM has properties of living systems - self-assembling, self-healing, and dynamic responsiveness - that many have attempted to artificially emulate in synthetic materials. We consider ELMs to be an emerging branch of biohybrid devices, with the distinction that the acellular components of the ELMs are either created wholly in situ or modified by the cells. The intimate integration of a living system into these materials allows our current efforts at engineering synthetic biological circuits to be applied towards materials science platforms. From the technologies reviewed herein, we can see two parallel efforts that converge under the definition of ELM efforts, as we have defined.

One effort is a bottom-up approach, attempting to use programmed cells as bionanomaterials factories for materials production. This approach draws heavily from synthetic biology and molecular self-assembly. All of the ELM efforts utilizing this bottom-up approach have focused on bacterial production of self-assembling extracellular materials, shadowing the dominance of prokaryotic systems in synthetic biology due to their relative simplicity and ease of growth. We believe that this focus on simpler bacterial systems will continue in the immediate future, as more researchers develop engineered cellular systems to secrete naturally occurring or synthetic biopolymers for extracellular assembly. One area that is ripe for exploration is the use of eukaryotic cells for generating ELMs, which has vast potential, given the spectrum of natural materials routinely used in everyday life that is derived from eukaryotic tissue -cotton fabric, leather, and wood. Concomitant with these efforts, more synthetic biological circuits will be integrated to control the production (and degradation) of ELMs spatially and temporally. Although these systems could theoretically recapitulate the complex materials-generative capability of natural systems, such as the wonderful example of trees, there is a substantial lack of engineering principles that adeptly weaves structural morphology and self-assembly together throughout multiple hierarchical scales.

The other approach in developing ELM technologies we have seen utilizes top-down assembly and is derived from synthetic materials research. Many of the composite ELM materials we have covered here are biohybrid materials/devices in which synthetic inorganic components (polymers, carbon-based and non-carbon based) compose a large part of the material. We attempted to include only those examples in which the living component substantially modified the materials or acted dynamically with the scaffolding to imbue the ELM with emergent function. These approaches attempt to expand the function of synthetic 
materials by the inclusion of living cells, which brings many of these examples into the realm of ELMs. These examples circumvent the need for cell-mediated organization by employing top-down engineering to place and precisely integrate the cells into the materials. Although this adds nontrivial cost and labor into the process, it enables the development complex functional materials that are currently unattainable from wholly bottom-up approaches. A significant benefit from these composite ELMs will be their inclusion in large-scale manufacturing of ELMs.

A review of traditional biomanufacturing processes that would be amenable to biomass isolation was presented for simpler materials, but we also highlighted cell-encapsulated nanofibers and 3D-printed cellular structures as key forward-looking manufacturing technologies that will likely play large roles in pushing the development of large-scale ELMs. It should be noted, however, that the few commercial examples of large-scale ELMs that we reviewed employed various manufacturing processes, many of them quite straightforward.

We anticipate the two approaches for engineering ELMs will continue, and we expect to see merging efforts as synthetic biology and synthetic materials efforts become more overlapping and enmeshed. As these two engineering efforts combine, ELM systems will rapidly emerge as a viable route for the development of programmable matter. One exciting area that we have touched upon in our review is the nascent field of synthetic morphogenesis, the fruits of which will guide us towards engineering living materials that are autonomously self-organizing spatially and temporally at multiple dimensions. The ongoing progress in these fields will drive the future of ELM design and engineering, towards the development of large-scale genetically programmable matter.

\section{References}

[1]. Chen X, Mahadevan L, Driks A, Sahin O, Nat. Nanotechnol. 2014, 9, 137. [PubMed: 24463362]

[2]. Benam KH, Villenave R, Lucchesi C, Varone A, Hubeau C, Lee H-H, Alves SE, Salmon M, Ferrante TC, Weaver JC, Bahinski A, Hamilton GA, Ingber DE, Nat. Methods 2015, 13, 151. [PubMed: 26689262]

[3]. Herland A, van der Meer AD, FitzGerald EA, Park T-E, Sleeboom JJF, Ingber DE, PLoS One 2016, 11, e0150360. [PubMed: 26930059]

[4]. Jain A, Barrile R, van der Meer AD, Mammoto A, Mammoto T, De Ceunynck K, Aisiku O, Otieno MA, Louden CS, Hamilton GA, Flaumenhaft R, Ingber DE, Clin. Pharmacol. Ther. 2017, 10.1002/cpt.742.

[5]. Kim HJ, Li H, Collins JJ, Ingber DE, Proc. Natl. Acad. Sci. U.S.A. 2016, 113, E7. [PubMed: 26668389]

[6]. Villenave R, Wales SQ, Hamkins-Indik T, Papafragkou E, Weaver JC, Ferrante TC, Bahinski A, Elkins CA, Kulka M, Ingber DE, PLoS One 2017, 12, e0169412. [PubMed: 28146569]

[7]. Carlsen RW, Edwards MR, Zhuang J, Pacoret C, Sitti M, Bibette J, Ai S, Wang J, Park J, Park S, Park S, Lab on a Chip 2014, 14, 3850. [PubMed: 25120224]

[8]. Liu X, Tang T-C, Tham E, Yuk H, Lin S, Lu TK, Zhao X, Proc. Natl. Acad. Sci. U.S.A. 2017, 114, 2200. [PubMed: 28202725]

[9]. Townsend-Nicholson A, Jayasinghe SN, Biomacromolecules 2006, 10.1021/BM060649H.

[10]. Liu Y, Rafailovich MH, Malal R, Cohn D, Chidambaram D, Proc. Natl. Acad. Sci. U.S.A. 2009, 106, 14201. [PubMed: 19667172] 
[11]. Ang HY, Irvine SA, Avrahami R, Sarig U, Bronshtein T, Zussman E, Boey FYC, Machluf M, Venkatraman SS, Biomatter 2014, 4, e28238. [PubMed: 24553126]

[12]. Dandoy P, Meunier CF, Leroux G. g., Voisin V, Giordano L, Caron N, Michiels C, Su B-L, PLoS One 2013, 8, e54683. [PubMed: 23372752]

[13]. Nassif N, Bouvet O, Noelle Rager M, Roux C. c., Coradin T, Livage J, Nat. Mater. 2002, 1, 42. [PubMed: 12618847]

[14]. Gullo MR, Takeuchi S, Paul O, Adv. Healthcare Mater. 2017, 6, 1601053.

[15]. Branda SS, Vik S, Friedman L, Kolter R, Trends Microbiol. 2005, 13, 20. [PubMed: 15639628]

[16]. Bazaka K, Crawford RJ, Nazarenko EL, Ivanova EP, in Bacterial Adhesion: Chemistry, Biology and Physics, 10.1007/978-94-007-0940-9_13 (Eds: Linke D, Goldman A), Springer Netherlands, Dordrecht 2011, 213.

[17]. Limoli DH, Jones CJ, Wozniak DJ, Microbiol. Spectrum 2015, 3.

[18]. Yi W, Liu X, Li Y, Li J, Xia C, Zhou G, Zhang W, Zhao W, Chen X, Wang PG, Proc. Natl. Acad. Sci. U.S.A. 2009, 106, 4207. [PubMed: 19251666]

[19]. Wratil PR, Horstkorte R, Reutter W, Angew. Chem. Int. Ed. 2016, 55, 9482.

[20]. Hsieh YC, Yano H, Nogi M, Eichhorn SJ, Cellulose 2008, 15, 507.

[21]. Lee KY, Buldum G, Mantalaris A, Bismarck A, Macromol. Biosci. 2014, 14, 10. [PubMed: 23897676]

[22]. Florea M, Reeve B, Abbott J, Freemont PS, Ellis T, Sci. Rep. 2016, 6, 23635. [PubMed: 27010592]

[23]. Brown AJ, J. Chem. Soc,Trans. 1886, 49, 432.

[24]. Ng FMC, Wang PW, The Design Journal 2016, 19, 837.

[25]. Florea M, Hagemann H, Santosa G, Abbott J, Micklem CN, Spencer-Milnes X, de Arroyo Garcia L, Paschou D, Lazenbatt C, Kong D, Chughtai H, Jensen K, Freemont PS, Kitney R, Reeve B, Ellis T, Proc. Natl. Acad. Sci. U.S.A. 2016, 113, E3431. [PubMed: 27247386]

[26]. Mangayil R, Rajala S, Pammo A, Sarlin E, Luo J, Santala V, Karp M, Tuukkanen S, ACS Appl Mater Interfaces 2017, 9, 19048. [PubMed: 28520408]

[27]. Yu J, Huang TR, Lim ZH, Luo R, Pasula RR, Liao LD, Lim S, Chen CH, Adv. Healthcare Mater. 2016, 5, 2983.

[28]. Fang J, Kawano S, Tajima K, Kondo T, Biomacromolecules 2015, 16, 3154. [PubMed: 26360299]

[29]. Sleytr UB, Messner P, Pum D, Sara M, Mol. Microbiol. 1993, 10, 911. [PubMed: 7934867]

[30]. Sleytr UB, Beveridge TJ, Trends Microbiol. 1999, 7, 253. [PubMed: 10366863]

[31]. Sleytr UB, Messner P, Pum D, Sara M, Angew. Chem. Int. Ed. 1999, 38, 1034.

[32]. Beveridge TJ, Graham LL, Microbiol. Mol. Biol. Rev. 1991, 55, 684.

[33]. Sleytr UB, Huber C, Ilk N, Pum D, Schuster B, Egelseer EM, FEMS Microbiol. Lett. 2007, 267, 131. [PubMed: 17328112]

[34]. Michon C, Langella P, Eijsink VG, Mathiesen G, Chatel JM, Microb. Cell Fact. 2016, 15, 70. [PubMed: 27142045]

[35]. Morais S, Shterzer N, Lamed R, Bayer EA, Mizrahi I, Biotechnol. Biofuels 2014, 7, 112. [PubMed: 25788977]

[36]. Dueholm MS, Albertsen M, Otzen D, Nielsen PH, PLoS One 2012, 7, e51274. [PubMed: 23251478]

[37]. Chen AY, Deng Z, Billings AN, Seker UO, Lu MY, Citorik RJ, Zakeri B, Lu TK, Nat. Mater. 2014, 13, 515. [PubMed: 24658114]

[38]. Abdelwahab MT, Kalyoncu E, Onur T, Baykara MZ, Seker UOS, Langmuir 2017, 33, 4337. [PubMed: 28388843]

[39]. Nguyen PQ, Botyanszki Z, Tay PK, Joshi NS, Nat. Commun. 2014, 5, 4945. [PubMed: 25229329]

[40]. Botyanszki Z, Tay PK, Nguyen PQ, Nussbaumer MG, Joshi NS, Biotechnol. Bioeng. 2015, 112, 2016. [PubMed: 25950512]

Adv Mater. Author manuscript; available in PMC 2019 May 01. 
[41]. Nussbaumer MG, Nguyen PQ, Tay PKR, Naydich A, Hysi E, Botyanszki Z, Joshi NS, ChemCatChem 2017, 10.1002/cctc.201701221,n/a.

[42]. Seker UO, Chen AY, Citorik RJ, Lu TK, ACS Synth. Biol. 2017, 6, 266. [PubMed: 27794590]

[43]. Ebuzer Kalyoncu REA, Tolga T Olmez and Urartu Ozgur Safak Seker, RSC Adv. 2017, 7, 32543.

[44]. Dorval Courchesne N-M, Duraj-Thatte A, Tay PKR, Nguyen PQ, Joshi NS, ACS Biomater. Sci. Eng. 2016, 3, 733.

[45]. Tay PKR, Nguyen PQ, Joshi NS, ACS Synth. Biol. 2017, 10.1021/acssynbio.7b00137.

[46]. Gilbert C, Howarth M, Harwood CR, Ellis T, ACS Synth. Biol. 2017, 6, 957. [PubMed: 28230977]

[47]. Ma Q, Yang Z, Pu M, Peti W, Wood TK, Environ. Microbiol. 2011, 13, 631. [PubMed: 21059164]

[48]. Lee J, Jayaraman A, Wood TK, BMC Microbiol. 2007, 7, 42. [PubMed: 17511876]

[49]. Hong SH, Hegde M, Kim J, Wang X, Jayaraman A, Wood TK, Nat. Commun. 2012, 3, 613. [PubMed: 22215088]

[50]. Hong SH, Lee J, Wood TK, Microb. Biotechnol. 2010, 3, 717. [PubMed: 21255366]

[51]. Wood TL, Guha R, Tang L, Geitner M, Kumar M, Wood TK, Proc. Natl. Acad. Sci. U.S.A. 2016, 113, E2802. [PubMed: 27140616]

[52]. Tecon R, Or D, Sci. Rep. 2017, 7, 43726. [PubMed: 28262696]

[53]. Duprey A, Chansavang V, Fremion F, Gonthier C, Louis Y, Lejeune P, Springer F, Desjardin V, Rodrigue A, Dorel C, J. Biol. Eng. 2014, 8, 19. [PubMed: 25104972]

[54]. Halan B, Buehler K, Schmid A, Trends Biotechnol. 2012, 30, 453. [PubMed: 22704028]

[55]. Singh R, Paul D, Jain RK, Trends Microbiol. 2006, 14, 389. [PubMed: 16857359]

[56]. Achal V, Mukherjee A, Kumari D, Zhang Q, Earth-Sci. Rev. 2015, 148, 1.

[57]. Bertucci A, Moya A, Tambutté S, Allemand D, Supuran CT, Zoccola D, Biorg. Med. Chem. 2013, 21, 1437.

[58]. Amao Y, Shuto N, Furuno K, Obata A, Fuchino Y, Uemura K, Kajino T, Sekito T, Iwai S, Miyamoto Y, Matsuda M, Faraday Discuss. 2012, 155, 289. [PubMed: 22470981]

[59]. Liu X, Tang TC, Tham E, Yuk H, Lin S, Lu TK, Zhao X, Proc. Natl. Acad. Sci. U.S.A. 2017, 114, 2200. [PubMed: 28202725]

[60]. Eroglu E, Agarwal V, Bradshaw M, Chen X, Smith SM, Raston CL, Swaminathan Iyer K, Green Chem. 2012, 14, 2682.

[61]. Cheng Z, Lu L, Kennes C, Ye J, Yu J, Chen D, Chen J, Bioresour. Technol. 2016, 218, 751. [PubMed: 27423036]

[62]. Gerber LC, Koehler FM, Grass RN, Stark WJ, Angew. Chem. Int. Ed. 2012, 51, 11293.

[63]. Gerber LC, Koehler FM, Grass RN, Stark WJ, Proc. Natl. Acad. Sci. U.S.A. 2012, 109, 90. [PubMed: 22198770]

[64]. Chen X, Mahadevan L, Driks A, Sahin O, Nat Nano 2014, 9, 137.

[65]. Chen X, Goodnight D, Gao Z, Cavusoglu AH, Sabharwal N, DeLay M, Driks A, Sahin O, Nat. Commun. 2015, 6, 7346. [PubMed: 26079632]

[66]. Wang W, Yao L, Cheng C-Y, Zhang T, Atsumi H, Wang L, Wang G, Anilionyte O, Steiner H, Ou J, Zhou K, Wawrousek C, Petrecca K, Belcher AM, Karnik R, Zhao X, Wang DIC, Ishii H, Sci. Adv. 2017, 3.

[67]. Zhang Q, Chen B, Tao L, Yan M, Chen L, Wei Y, RSC Adv. 2014, 4, 32475.

[68]. Magdanz V, Sanchez S, Schmidt OG, Adv. Mater. 2013, 25, 6581. [PubMed: 23996782]

[69]. Williams BJ, Anand SV, Rajagopalan J, Saif MT, Nat. Commun. 2014, 5, 3081. [PubMed: 24435099]

[70]. Feinberg AW, Feigel A, Shevkoplyas SS, Sheehy S, Whitesides GM, Parker KK, Science 2007, 317, 1366. [PubMed: 17823347]

[71]. Nawroth JC, Lee H, Feinberg AW, Ripplinger CM, McCain ML, Grosberg A, Dabiri JO, Parker KK, Nat. Biotechnol. 2012, 30, 792. [PubMed: 22820316]

[72]. Park S-J, Gazzola M, Park KS, Park S, Di Santo V, Blevins EL, Lind JU, Campbell PH, Dauth S, Capulli AK, Pasqualini FS, Ahn S, Cho A, Yuan H, Maoz BM, Vijaykumar R, Choi J-W, 
Deisseroth K, Lauder GV, Mahadevan L, Parker KK, Science 2016, 353, 158. [PubMed: 27387948]

[73]. Chan V, Park K, Collens MB, Kong H, Saif TA, Bashir R, Sci. Rep. 2012, 2, 857. [PubMed: 23155480]

[74]. Cvetkovic C, Raman R, Chan V, Williams BJ, Tolish M, Bajaj P, Sakar MS, Asada HH, Saif MTA, Bashir R, Proc. Natl. Acad. Sci. U.S.A. 2014, 111, 10125. [PubMed: 24982152]

[75]. Verlinden RAJ, Hill DJ, Kenward MA, Williams CD, Radecka I, J. Appl. Microbiol. 2007, 102, 1437. [PubMed: 17578408]

[76]. Chuah J-A, Yamada M, Taguchi S, Sudesh K, Doi Y, Numata K, Polym. Degrad. Stab. 2013, 98.

[77]. Lee W-H, Loo C-Y, Nomura CT, Sudesh K, Bioresour. Technol. 2008, 99.

[78]. Park S, Lee T, Lim S-C, Kim T, Lee H, Kim M, Lee S, Song B, Appl. Microbiol. Biotechnol. 2012, 93.

[79]. Insomphun C, Kobayashi S, Fujiki T, Numata K, AMB Express 2016, 6, 29. [PubMed: 27075993]

[80]. Li Z, Yang J, Loh XJ, NPG Asia Mater. 2016, 8, e265.

[81]. Arshad A, Ashraf B, Ali I, Jamil N, Front. Biol. 2017, 12, 210.

[82]. Magennis EP, Fernandez-Trillo F, Sui C, Spain SG, Bradshaw DJ, Churchley D, Mantovani G, Winzer K, Alexander C, Nat. Mater. 2014, 13, 748. [PubMed: 24813421]

[83]. Valentini L, Bon SB, Pugno NM, ACS Appl. Mater. Interfaces 2016, 8, 22714. [PubMed: 27529441]

[84]. Valentini L, Bittolo Bon S, Signetti S, Pugno NM, ACS Appl. Mater. Interfaces 2016, 8, 7607. [PubMed: 26971362]

[85]. Valentini L, Bon SB, Signetti S, Tripathi M, Iacob E, Pugno NM, Sci. Rep. 2016, 6, 27031. [PubMed: 27279425]

[86]. Valentini L, Bittolo Bon S, Pugno NM, Adv. Funct. Mater. 2017, 27, 1606526.

[87]. Yuan Y, Zhou S, Yang G, Yu Z, RSC Adv. 2013, 3, 18844.

[88]. Zhao C.-e., Wu J, Ding Y, Wang VB, Zhang Y, Kjelleberg S, Loo JSC, Cao B, Zhang Q, ChemElectroChem 2015, 2, 654.

[89]. Zhou L, Fu P, Wang Y, Sun L, Yuan Y, J. Mater. Chem. A 2016, 4, 7222.

[90]. Perry CC, Keeling-Tucker T, J. Biol. Inorg. Chem. 2000, 5, 537. [PubMed: 11085644]

[91]. Trevors JT, Antonie Van Leeuwenhoek 1997, 71, 271. [PubMed: 9111922]

[92]. Phoenix VR, Adams DG, Konhauser KO, Chem. Geol. 2000, 169, 329.

[93]. Hirota R, Hata Y, Ikeda T, Ishida T, Kuroda A, Bacteriol J. 2010, 192, 111.

[94]. Benning LG, Phoenix VR, Yee N, Konhauser KO, Geochim. Cosmochim. Acta 2004, 68, 743.

[95]. Tubaña BS, Heckman JR, in Silicon and Plant Diseases, 10.1007/978-3-319-22930-0_2 (Eds:

Rodrigues FA, Datnoff LE), Springer International Publishing, Cham 2015, 7.

[96]. Price CT, Koval KJ, Langford JR, Int. J. Endocrinol. 2013, 2013, 6.

[97]. Meunier CF, Dandoy P, Su BL, J. Colloid Interface Sci. 2010, 342, 211. [PubMed: 19944428]

[98]. Poulsen N, Chesley PM, Kröger N, J. Phycol. 2006, 42, 1059.

[99]. Poulsen N, Berne C, Spain J, Kröger N, Angew. Chem. Int. Ed. 2007, 46, 1843.

[100]. Sheppard V, Scheffel A, Poulsen N, Kröger N, Appl. Environ. Microbiol. 2012, 78, 211. [PubMed: 22057862]

[101]. Delalat B, Sheppard VC, Rasi Ghaemi S, Rao S, Prestidge CA, McPhee G, Rogers M-L, Donoghue JF, Pillay V, Johns TG, Kröger N, Voelcker NH, Nat. Commun. 2015, 6, 8791. [PubMed: 26556723]

[102]. Jonkers HM, HERON 2011, 56, 1.

[103]. Jonkers HM, Schlangen E, Proceedings of FRACOS6: fracture mechanics of concrete and concrete structures Catania, Italy 2007, 1821.

[104]. Jonkers HM, Thijssen A, Muyzer G, Copuroglu O, Schlangen E, Ecol. Eng. 2010, 36, 230.

[105]. Wang J, Van Tittelboom K, De Belie N, Verstraete W, Constr. Build. Mater. 2012, 26, 532.

Adv Mater. Author manuscript; available in PMC 2019 May 01. 
[106]. de Koster SAL, Mors RM, Nugteren HW, Jonkers HM, Meesters GMH, van Ommen JR, Procedia Eng. 2015, 102, 475.

[107]. Weyant C, Athalye V, Ragavan S, Rajarathnam U, Lalchandani D, Maithel S, Baum E, Bond TC, Environ. Sci. Technol. 2014, 48, 6477. [PubMed: 24735080]

[108]. Raut SH, Sarode DD, Lele SS, World J Microbiol. Biotechnol. 2014, 30, 191.

[109]. Nave K, Vol. 2017, WIRED UK, WIRED UK 2016.

[110]. Das AAK, Bovill J, Ayesh M, Stoyanov SD, Paunov VN, Journal of Materials Chemistry B 2016, 4, 3685.

[111]. Vickers D, Modern Design 2008, 63.

[112]. Ludwig F, de Bruyn G, Thielen M, Speck T, "Plant stems as building material for living plant constructions", presented at UMR EcoFoG, 6th Plant Biomechanics Conference, Cayenne, French Guiana, France, 2009.

[113]. Ludwig F, Storz O, Schwertfeger H, Architectural Design 2012, 82, 82.

[114]. Harfouche A, Meilan R, Altman A, Trends Biotechnol. 2011, 29, 9. [PubMed: 20970211]

[115]. Mathews JH, Campbell MM, Forestry 2000, 73, 371.

[116]. Bigall NC, Reitzig M, Naumann W, Simon P, van Pée K-H, Eychmüller A, Angew. Chem. Int. Ed. 2008, 120, 7994.

[117]. Rehman A, Majeed MI, Ihsan A, Hussain SZ, Saif ur R, Ghauri MA, Khalid ZM, Hussain I, J. Nanopart. Res. 2011, 13, 6747.

[118]. Haneef M, Ceseracciu L, Canale C, Bayer IS, Heredia-Guerrero JA, Athanassiou A, Sci. Rep. 2017, 7, 41292. [PubMed: 28117421]

[119]. Kozicki MN, Roberson RW, Whidden TK, Kersey SE, J. Vac. Sci. Technol. A 1995, 13, 1808.

[120]. Barrasa JM, Gutiérrez A, Escaso V, Guillén F, Martínez MJ, Martínez AT, Appl. Environ. Microbiol. 1998, 64, 325. [PubMed: 9435085]

[121]. Hietala AM, Nagy NE, Steffenrem A, Kvaalen H, Fossdal CG, Solheim H, Appl. Environ. Microbiol. 2009, 75, 4069. [PubMed: 19376909]

[122]. Nødvig CS, Nielsen JB, Kogle ME, Mortensen UH, Zou G, Nielsen KF, PLoS One 2015, 10, e0133085. [PubMed: 26177455]

[123]. Iguchi M, Yamanaka S, Budhiono A, J. Mater. Sci. 2000, 35, 261.

[124]. Vandamme EJ, De Baets S, Vanbaelen A, Joris K, De Wulf P, Polym. Degrad. Stab. 1998, 59, 93.

[125]. Shah J, Malcolm Brown R, Appl. Microbiol. Biotechnol. 2005, 66, 352. [PubMed: 15538556]

[126]. Li S, Huang D, Zhang B, Xu X, Wang M, Yang G, Shen Y, Adv. Energy Mater. 2014, 4, 1301655.

[127]. Wood TK, Hong SH, Ma Q, Trends Biotechnol. 2011, 29, 87. [PubMed: 21131080]

[128]. Florea M, Hagemann H, Santosa G, Abbott J, Micklem CN, Spencer-Milnes X, De L, Garcia A, Paschou D, Lazenbatt C, Kong D, Chughtai H, Jensen K, Freemont PS, Kitney R, Reeve B, Ellis T, Boeke JD, Proc. Natl. Acad. Sci. U.S.A. 2016, 113, E3431. [PubMed: 27247386]

[129]. Zhao C, Li Z, Li T, Zhang Y, Bryant DA, Zhao J, Cell Discovery 2015, 1, 15004. [PubMed: 27462405]

[130]. Al-Mashhadani MKH, Wilkinson SJ, Zimmerman WB, Chem. Eng. Sci. 2015, 137, 243.

[131]. Cheng H-P, Wang P-M, Chen J-W, Wu W-T, Biotechnol. Appl. Biochem. 2002, 35, 125. [PubMed: 11916454]

[132]. Willquist K, Pawar SS, Van Niel EWJ, Niel E. W. J. v., Kim IH, Yeom SH, Microb. Cell Fact. 2011, 10, 111. [PubMed: 22189215]

[133]. Zhang Z-P, Show K-Y, Tay J-H, Liang DT, Lee D-J, Energy Fuels 2008, 22, 87.

[134]. Morato J, Codony F, Mas J, Biofouling 2005, 21, 151. [PubMed: 16371335]

[135]. Junker LM, Toba FA, Hay AG, FEMS Microbiol. Lett. 2007, 268, 237. [PubMed: 17227468]

[136]. Matsumoto S, Ohtaki A, Hori K, Environ. Sci. Technol, 46, 10175.

[137]. Qureshi N, Annous BA, Ezeji TC, Karcher P, Maddox IS, Dasgupta M, Marrie TJ, Microb. Cell Fact. 2005, 4, 24. [PubMed: 16122390] 
[138]. Qureshi N, Schripsema J, Lienhardt J, Blaschek HP, World J Microbiol. Biotechnol. 2000, 16, 377.

[139]. Tse S-W, Yu J, Biofouling 2003, 19, 223. [PubMed: 14626842]

[140]. Lopez-Ponnada EV, Lynn TJ, Peterson M, Ergas SJ, Mihelcic JR, J. Biol. Eng. 2017, 11, 16. [PubMed: 28469703]

[141]. Zune Q, Delepierre A, Gofflot S, Bauwens J, Twizere JC, Punt PJ, Francis F, Toye D, Bawin T, Delvigne F, Appl. Microbiol. Biotechnol. 2015, 99, 6241. [PubMed: 25935344]

[142]. Warnock JN, Bratch K, Al-Rubeai M, in Bioreactors for Tissue Engineering, 10.1007/1-4020-3741-4_4, Springer-Verlag, Berlin/Heidelberg 2005, 87.

[143]. Hekmat D, Feuchtinger A, Stephan M, Vortmeyer D, Biodegradation 2004, 15, 133. [PubMed: 15068374]

[144]. Skybov T, P?ibyl M, Pocedi J, Hasal P, J. Biotechnol. 2012, 157, 512. [PubMed: 21930164]

[145]. Tay A, Yang ST, Biotechnol. Bioeng. 2002, 80, 1. [PubMed: 12209781]

[146]. Letnik I, Avrahami R, Rokem JS, Greiner A, Zussman E, Greenblatt C, Biomacromolecules 2015, 16, 3322. [PubMed: 26351729]

[147]. Knierim C, Enzeroth M, Kaiser P, Dams C, Nette D, Seubert A, Klingl A, Greenblatt CL, Jérôme V, Agarwal S, Freitag R, Greiner A, Macromol. Biosci. 2015, 15, 1052. [PubMed: 25845988]

[148]. Xie S, Tai S, Song H, Luo X, Zhang H, Li X, Journal of Materials Chemistry B 2016, 4, 6820.

[149]. Connell JL, Ritschdorff ET, Whiteley M, Shear JB, Proc. Natl. Acad. Sci. U.S.A. 2013, 110, 18380. [PubMed: 24101503]

[150]. Lehner BAE, Schmieden DT, Meyer AS, ACS Synth. Biol. 2017, 6, 1124. [PubMed: 28225616]

[151]. Homan KA, Kolesky DB, Skylar-Scott MA, Herrmann J, Obuobi H, Moisan A, Lewis JA, Sci. Rep. 2016, 6, 34845. [PubMed: 27725720]

[152]. Teague BP, Guye P, Weiss R, Cold Spring Harb. Perspect. Biol. 2016, 8.

[153]. Cachat E, Liu W, Hohenstein P, Davies JA, J. Biol. Eng. 2014, 8, 26. [PubMed: 25478005]

[154]. de Bono B, Grenon P, Baldock R, Hunter P, Journal of Biomedical Semantics 2013, 4, 22. [PubMed: 24103658]

[155]. Pavlicev M, Widder S, J. Exp. Zool. B Mol. Dev. Evol. 2015, 324, 104. [PubMed: 25755143]

[156]. Zartman JJ, Shvartsman SY, Annu. Rev. Chem. Biomol. Eng. 2010, 1, 231. [PubMed: 22432580]

[157]. Mohamadlou H, Podgorski GJ, Flann NS, BioSyst. 2016, 146, 77.

[158]. Mammoto T, Mammoto A, Ingber DE, Annu. Rev. Cell Dev. Biol. 2013, 29, 27. [PubMed: 24099083]

[159]. Scholes NS, Isalan M, Curr. Opin. Chem. Biol. 2017, 40, 1. [PubMed: 28463802]

[160]. Wolpert L, Curr. Top. Dev. Biol. 1971, 6, 183. [PubMed: 4950136]

[161]. Basu S, Gerchman Y, Collins CH, Arnold FH, Weiss R, Nature 2005, 434, 1130. [PubMed: 15858574]

[162]. Tabor JJ, Salis HM, Simpson ZB, Chevalier AA, Levskaya A, Marcotte EM, Voigt CA, Ellington AD, Cell 2009, 137, 1272. [PubMed: 19563759]

[163]. Liu C, Fu X, Liu L, Ren X, Chau CK, Li S, Xiang L, Zeng H, Chen G, Tang LH, Lenz P, Cui X, Huang W, Hwa T, Huang JD, Science 2011, 334, 238. [PubMed: 21998392]

[164]. Steinberg MS, Curr. Opin. Genet. Dev. 2007, 17, 281. [PubMed: 17624758]

[165]. Cachat E, Liu W, Martin KC, Yuan X, Yin H, Hohenstein P, Davies JA, Sci. Rep. 2016, 6, 20664. [PubMed: 26857385]

[166]. Rudge TJ, Federici F, Steiner PJ, Kan A, Haseloff J, ACS Synth. Biol. 2013, 2, 705. [PubMed: 23688051]

[167]. Ben-Jacob E, Schochet O, Tenenbaum A, Cohen I, Czirok A, Vicsek T, Nature 1994, 368, 46. [PubMed: 8107881]

[168]. Ben-Jacob E, Cohen II, Shochet O, Tenenbaum A, Czirok A, Vicsek T, Phys. Rev. Lett. 1995, 75, 2899. [PubMed: 10059433] 
[169]. Sanchez JA, Lasker HR, Nepomuceno EG, Sanchez JD, Woldenberg MJ, Am. Nat. 2004, 163, E24. [PubMed: 15026984]

[170]. Nieminen L, Webb S, Smith MC, Hoskisson PA, PLoS One 2013, 8, e54316. [PubMed: 23441147]

[171]. Asally M, Kittisopikul M, Rue P, Du Y, Hu Z, Cagatay T, Robinson AB, Lu H, Garcia-Ojalvo J, Suel GM, Proc. Natl. Acad. Sci. U.S.A. 2012, 109, 18891. [PubMed: 23012477]

[172]. Turing AM, Bull. Math. Biol. 1990, 52, 153. [PubMed: 2185858]

[173]. Rehm BHA, Nat. Rev. Microbiol. 2010, 8, 578. [PubMed: 20581859]

[174]. Kröger N, Curr. Opin. Chem. Biol. 2007, 11, 662. [PubMed: 17991447]

[175]. Taylor-Foster J, in ArchDaily, Vol. 2017, ArchDaily, http://www.archdaily.com/472905/bricksgrown-from-bacteria 2014. 


\section{Engineered Living Materials}

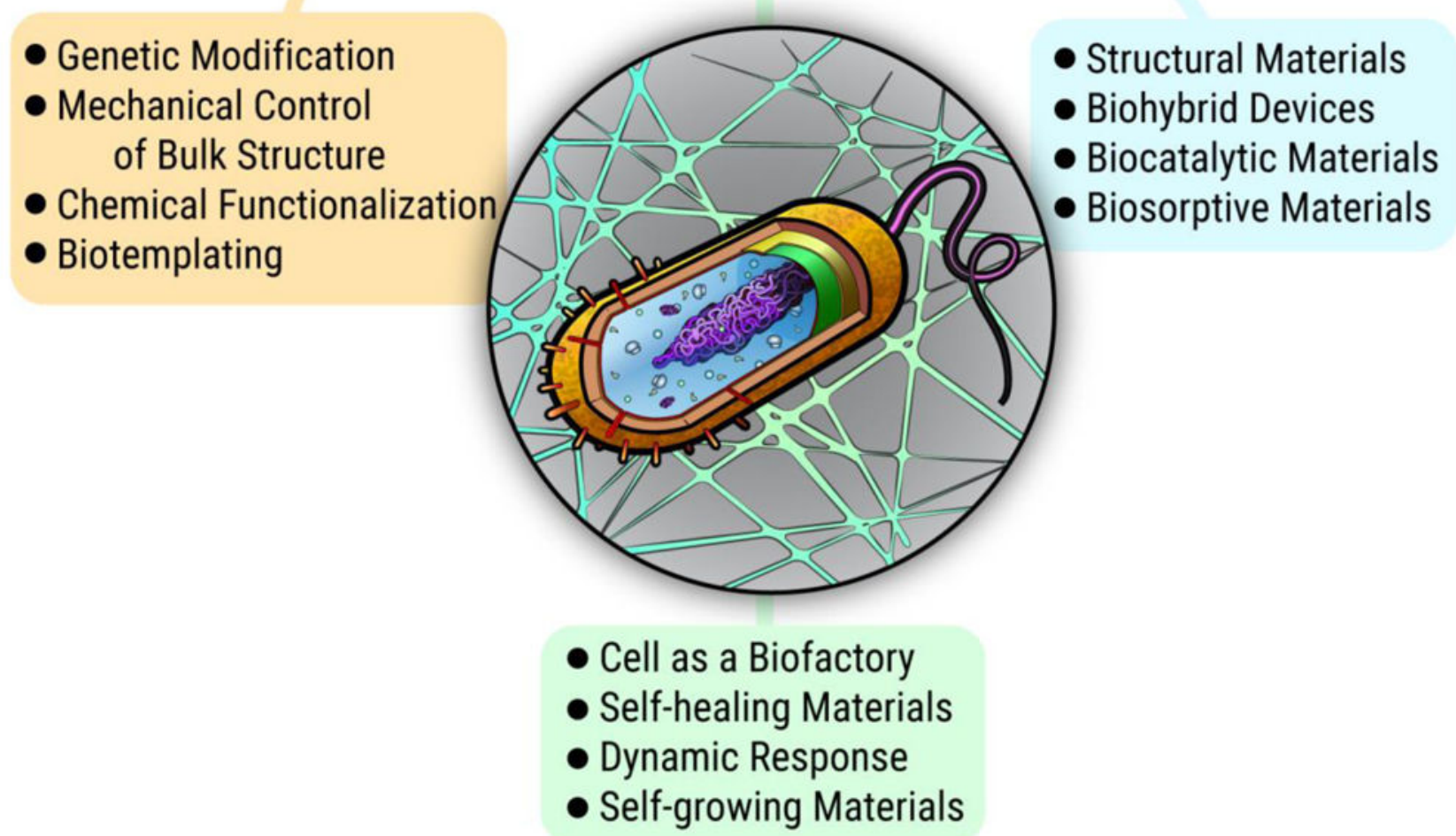

Figure 1. Properties of Engineered Living Materials.

ELMs leverage the adaptive responses, self-replicating potential, and robustness of biological systems for the programmed assembly and modulation of novel materials. The material can be engineered by genetic, spatial patterning, or chemical means, and can be designed for a wide variety of functions. These ELMs could be employed as micro to largescale structural materials, as a kind of biohybrid device that integrates synthetic materials with living systems, or for specialty applications such as biocatalysis or toxin sequestration. 

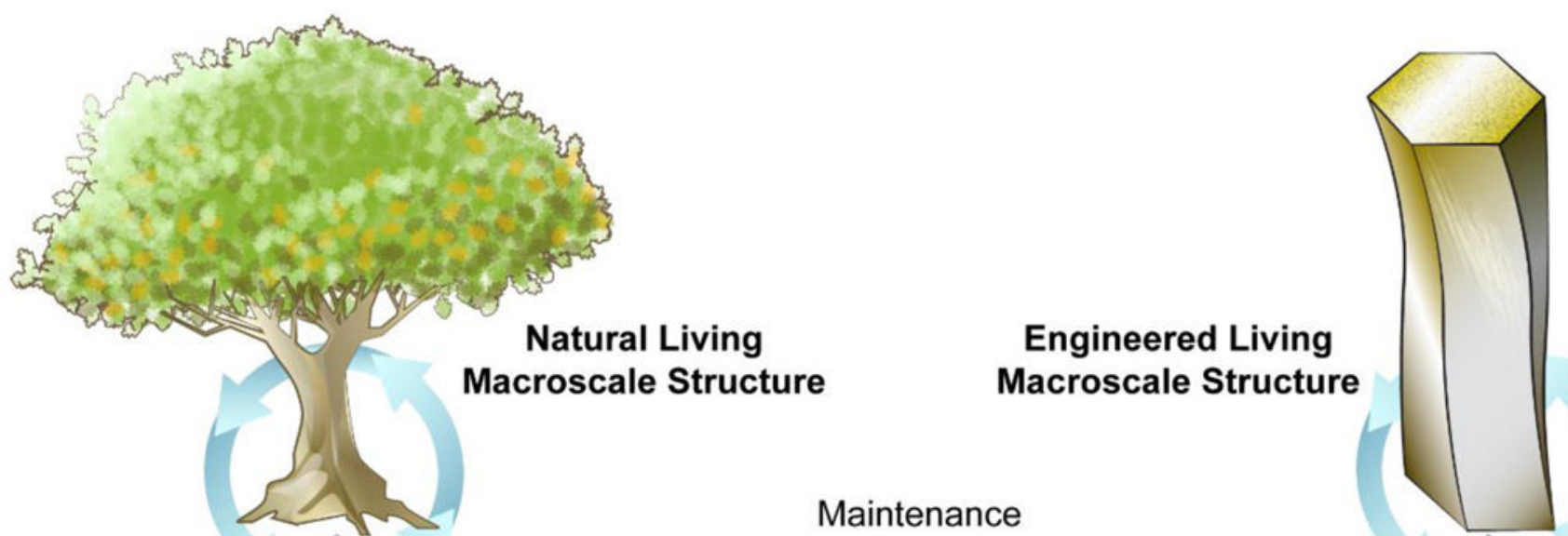

\section{Maintenance}

\& Renewal
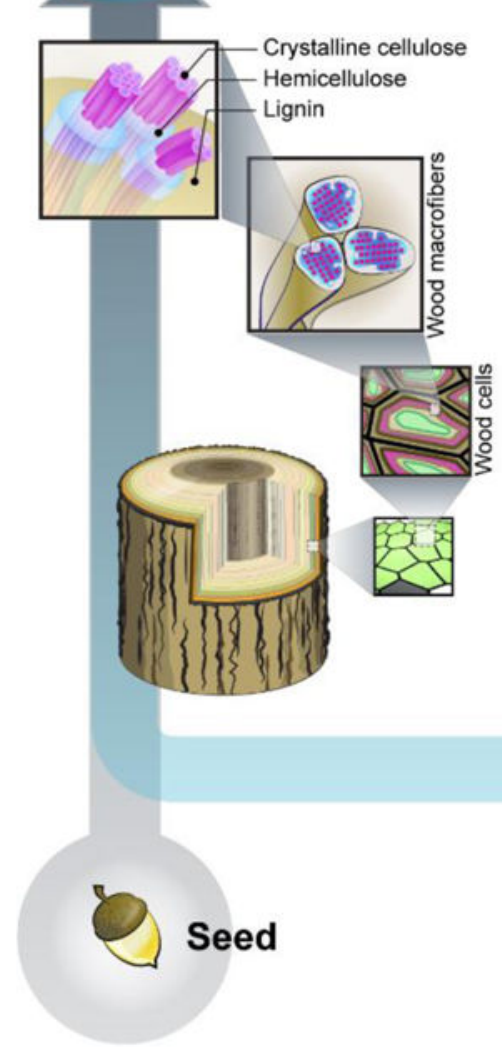

Self Organization

\&

Self Assembly

Morphogenesis
Water,

Energy Sources, Nutrients,

\&

Carbon Sources
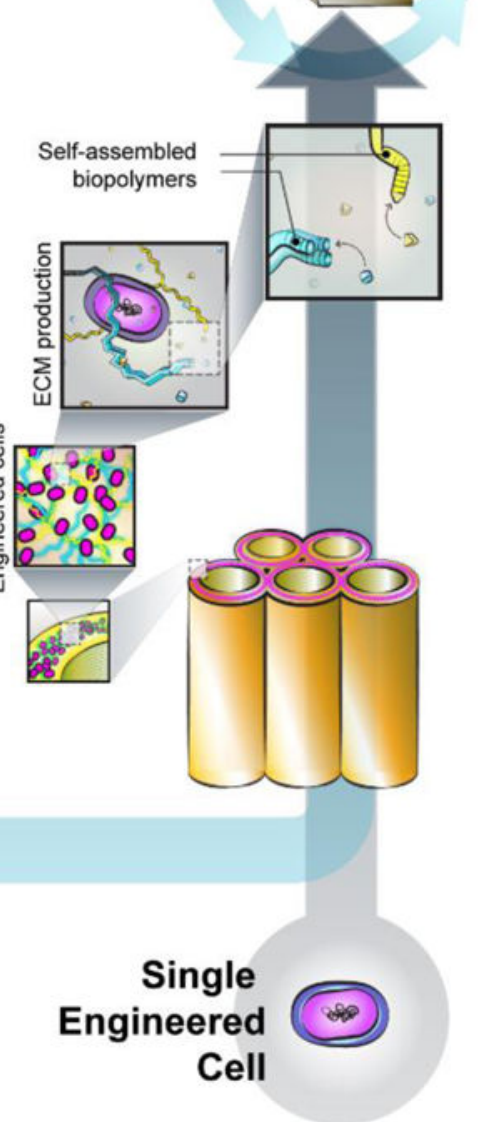

Figure 2. ELMs as Bioinspired Materials Engineering.

A single seed contains all the essential information needed to create a tree, and it does so by genetically encoding the metabolic functions required for acquiring energy (photosynthesis) and mechanisms for utilizing local resources (carbon dioxide and water). It also contains the information necessary for successful multi-scale hierarchical morphogensis based on principles of cellular self-organization and molecular self-assembly. These living structures are capable of dynamically responding to the environment and can maintain and renew themselves over time. Synthetic ELMs attempt to replicate these characteristics, by using synthetic biology to engineer cells to fabricate, assemble, and maintain autonomously 
produced materials composed of one or more biomaterials from a library of potential biomaterials. The cells could be metabolically engineered to utilize local energy sources and materials. By also incorporating programmed synthetic morphogenesis, large-scale structures can be designed with complex organization over multiple length scales. 
a b

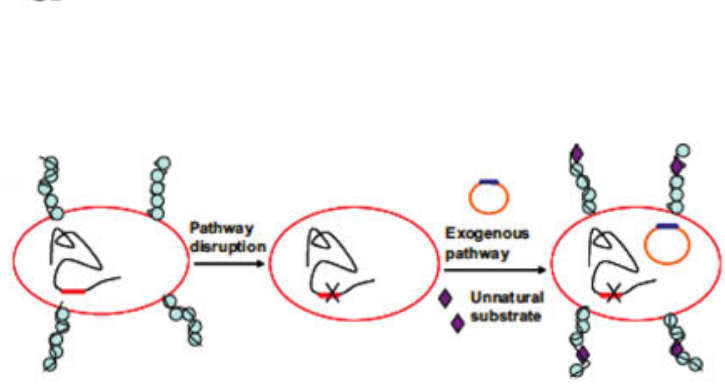

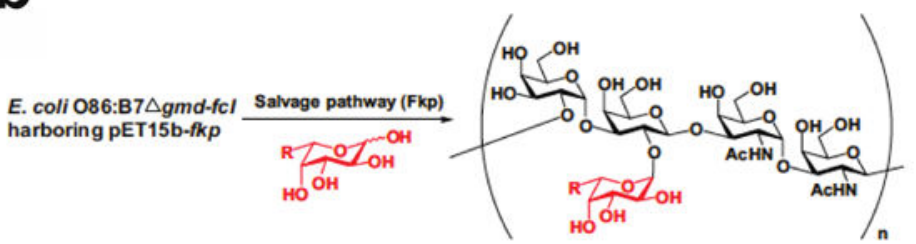

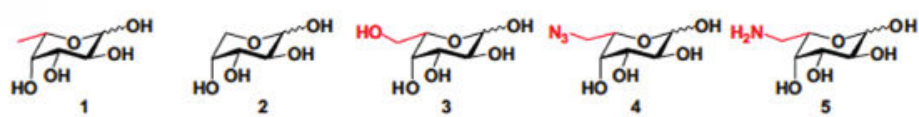

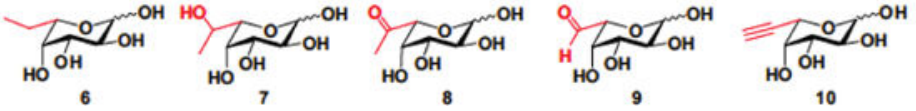

Figure 3. Engineering Bacterial Polysaccharides.

(a) Strategy for in vivo generation of modified polysaccharides by metabolic pathway engineering. (b) Generation of polysaccharides containing fucose analogs and their modifications located at C-6 position of fucose. Both images are from Yi, et al. ${ }^{[18]}$ 
a

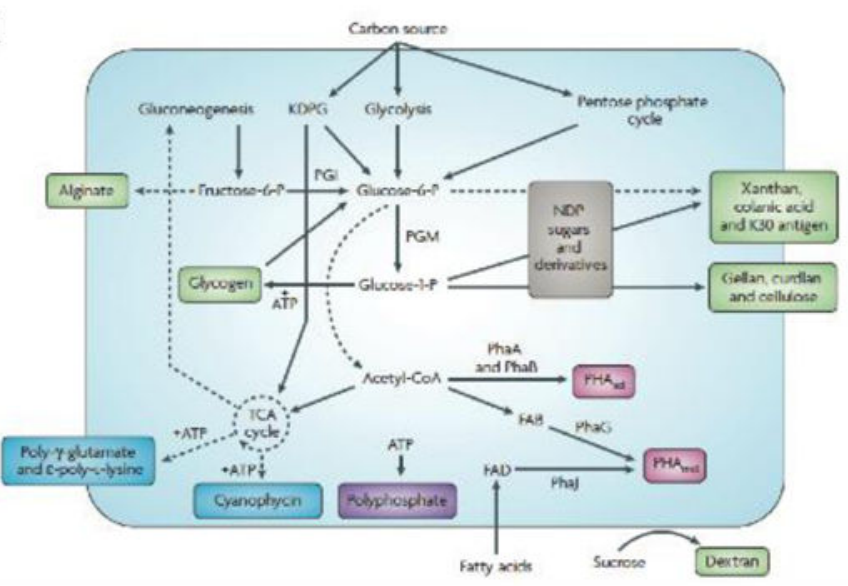

b
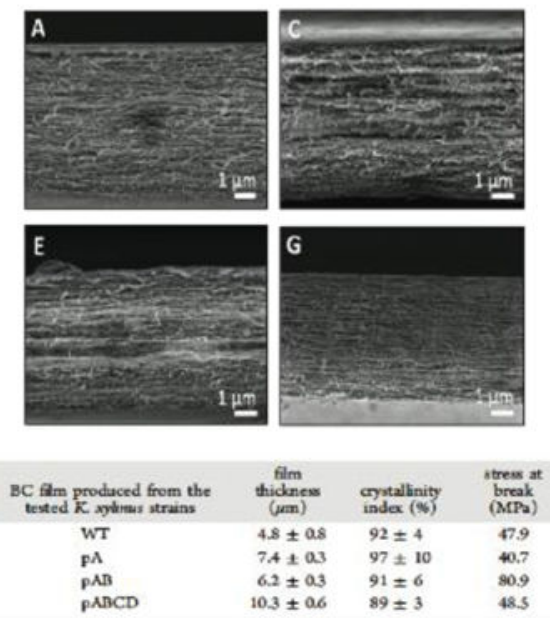

d
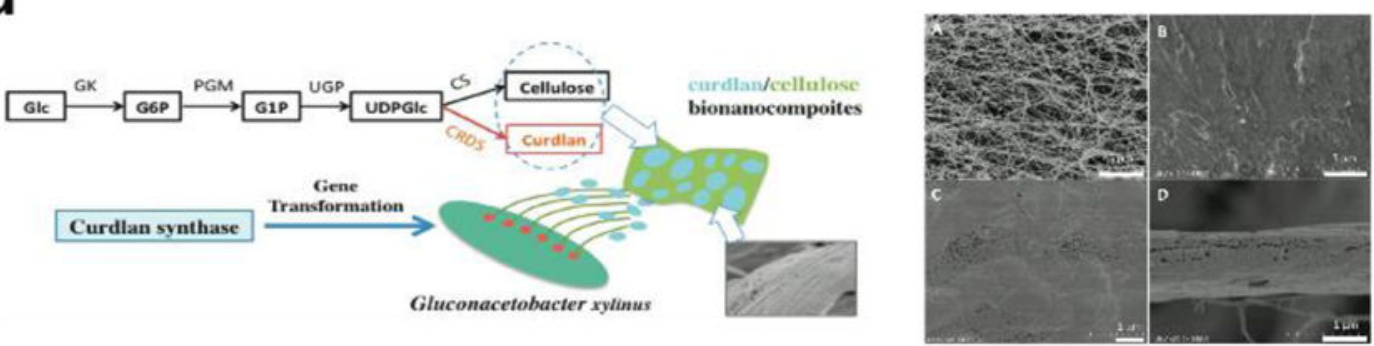

Figure 4. Engineered Polysaccharide-Based ECMs for Functional Biofilms.

(a) Bacterial polymer biosynthesis pathways from intermediates of central metabolism (polysaccharides shown in green). ${ }^{[173]}$ Cellulose (b-d). (b) Engineering the thickness and piezoelectricity of bacterial cellulose. ${ }^{[26]}$ (c) Engineering of spatial and temporal patterned and functionalized cellulose materials on a macroscale. ${ }^{[25]}$ (d) In vivo curdlan and cellulose bionanocomposite synthesis to modulate cellulose properties. ${ }^{[28]}$ 
a

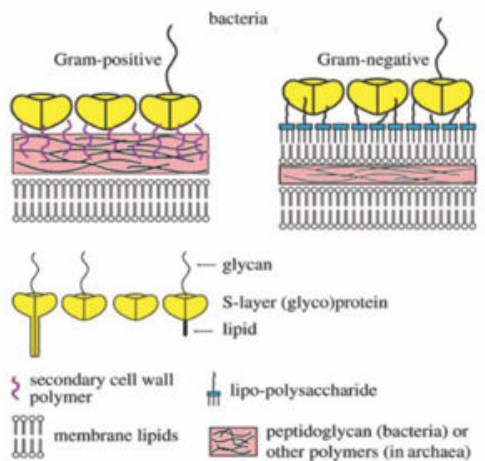

b
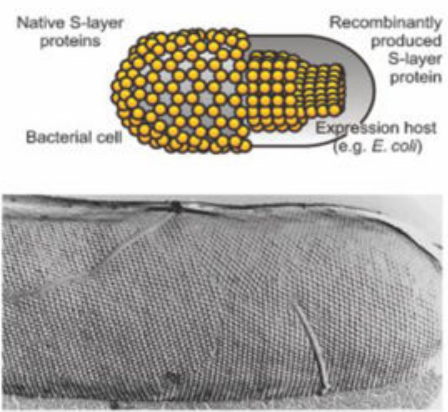

C d
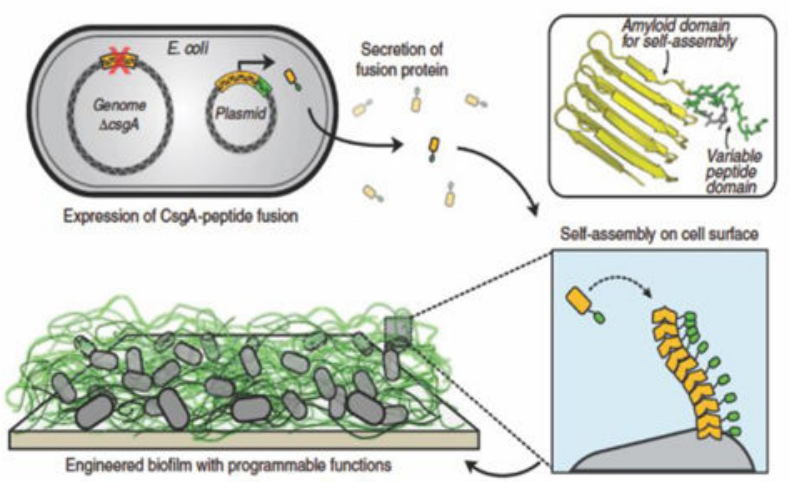

f
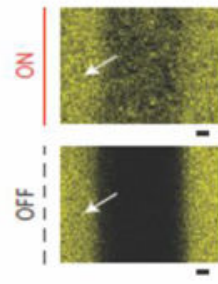

SEMVEDS
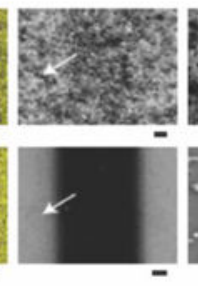

SEM
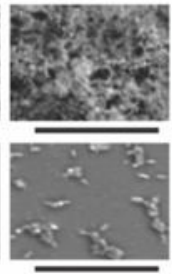
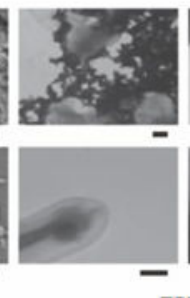
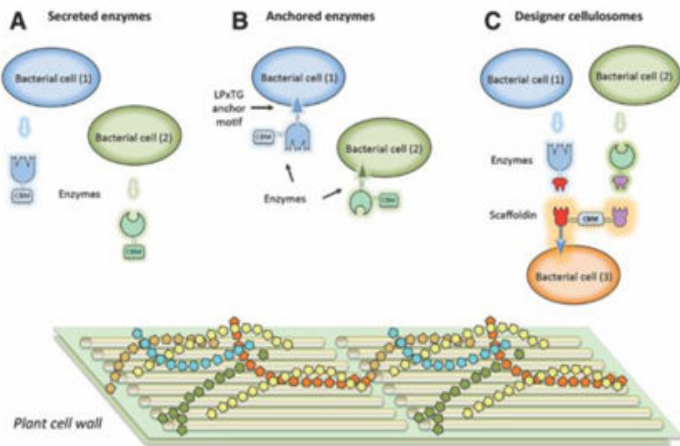

Figure 5. Engineered Protein-Based ECMs for Functional Biofilms.

S-layer proteins (a-b). (a) Schematic of S-layer proteins in gram positive and negative bacteria. ${ }^{[30]}$ (b) Scheme of S-layer protein on bacterial cell surface and SEM image of cell surface of Thermoanaerobacter thermohydrosulfuricus L111 showing a hexagonal (p6) surface lattice. ${ }^{[30]}$ (c) The construction of the three paradigmatic systems involved the division of the production and organization of the enzymes and scaffold proteins into different strains of L. plantarum. ${ }^{[35]}$ (d) Scheme of genetic programming and modularity of the BIND system. ${ }^{[39]}$ (e) Catalytic-BIND: biofilm functionalization with enzymes through 
the covalent modification of curli fibers. ${ }^{[40]}$ (f) SEM/EDS and TEM images of environmentally switchable conductive biofilms. ${ }^{[42]}$ (g) Conceptual depiction of selfregulating mercury binding circuit. ${ }^{[45]}$ 


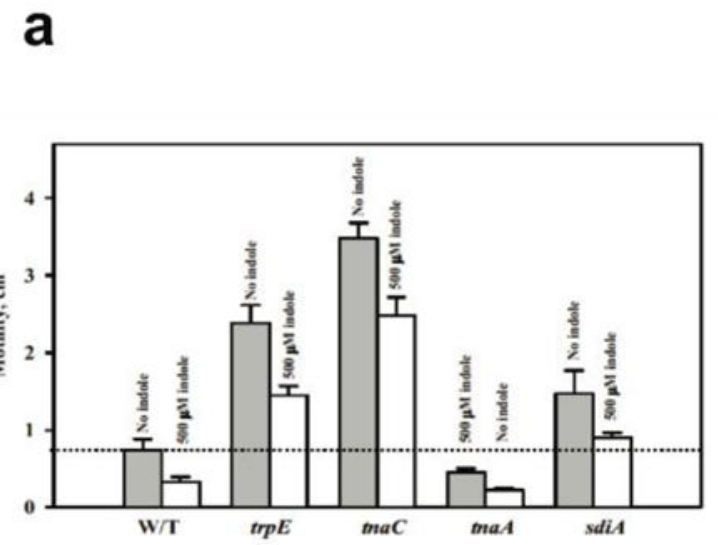

b
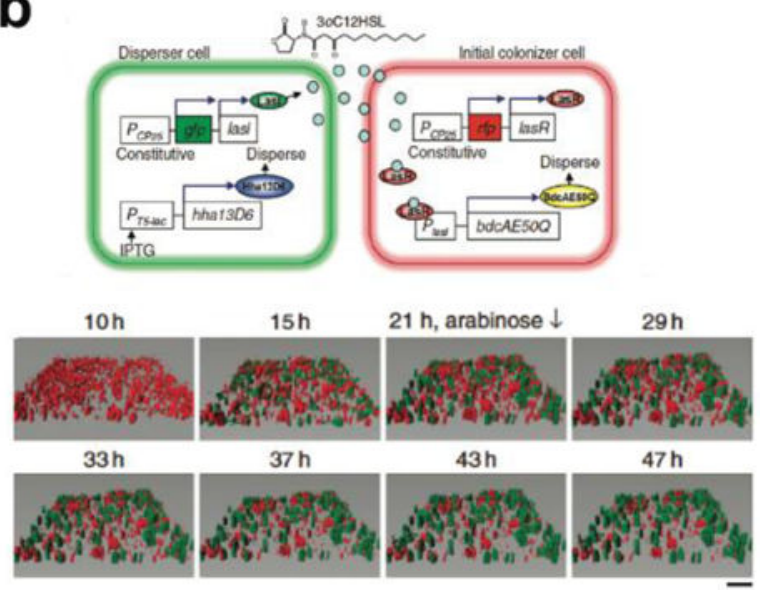

C
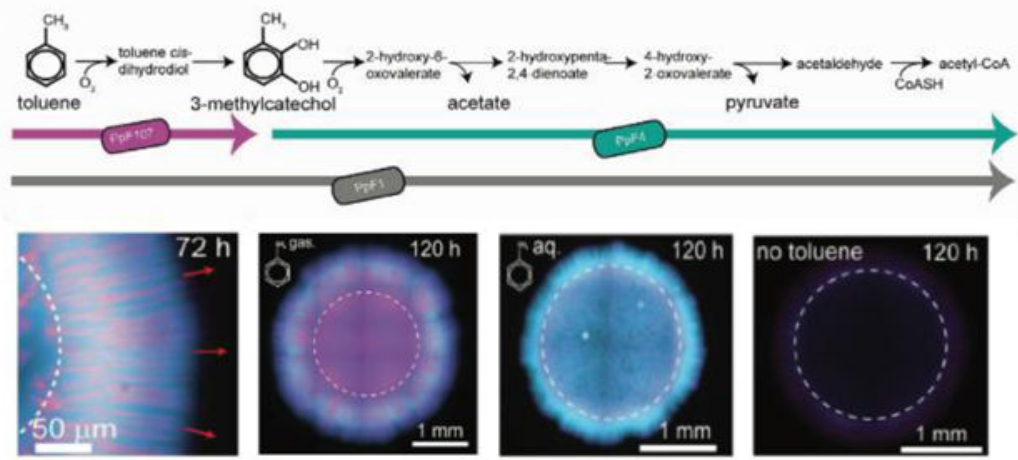

d
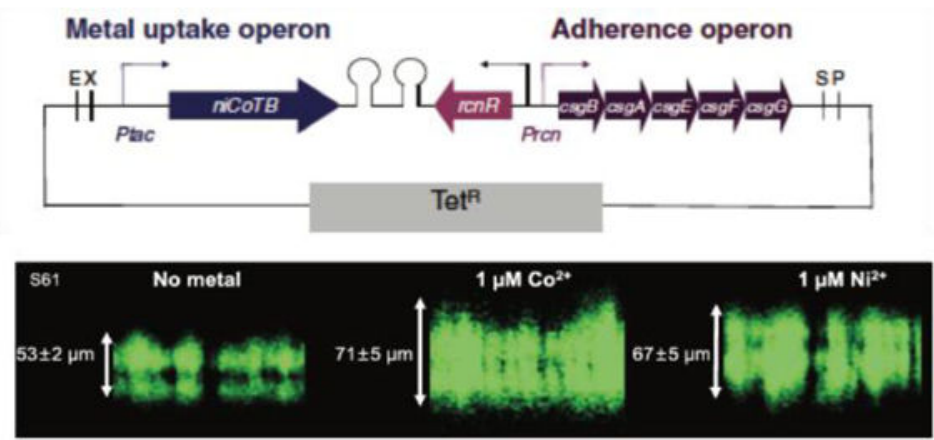

Figure 6. Engineering Bacteria to Control Biofilm Pattern Formation.

(a) Effect of indole $(500 \mu \mathrm{M})$ on the biofilm formation and motility of wild-type and engineered E. coli. ${ }^{[48]}$ (b) $\mu \mathrm{BE}$ metabolic circuit with the two E. coli cell types communicate by using the LasI / LasR QS module of $P$. aeruginosa (top) and dispersal of dual-species biofilms using arabinose (bottom). ${ }^{[47]}$ (c) Toluene degradation pathway from P. putida F1 and partial pathways carried out by mutant strains $\mathrm{PpF} 4$ and $\mathrm{PpF} 10732$ (top). Spatial patterns arising from the consortium of mutants (bottom). ${ }^{[52]}$ (d) Schematic representation of the engineered plasmid conferring constitutive metal uptake and inducible adhesion (top). 
Thickness of biofilm formed by the S61 strain in the presence or absence of cobalt and nickel (bottom). ${ }^{[53]}$ 
a
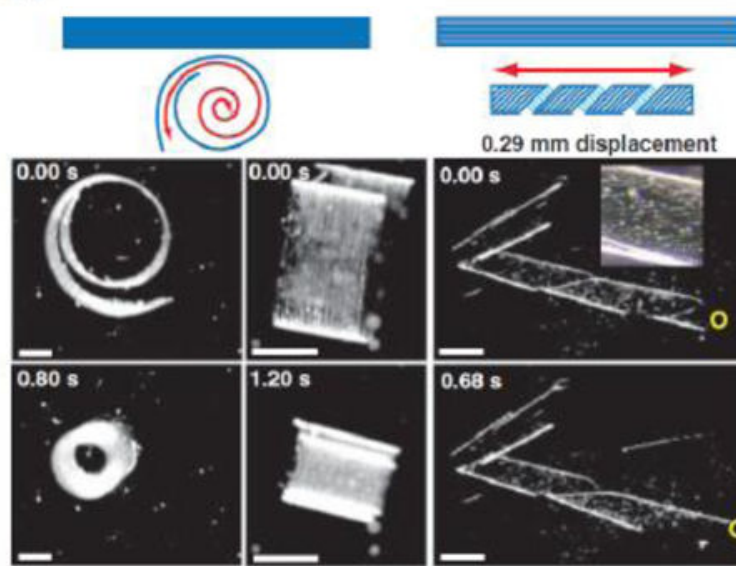
$0.00 \mathrm{~s}$
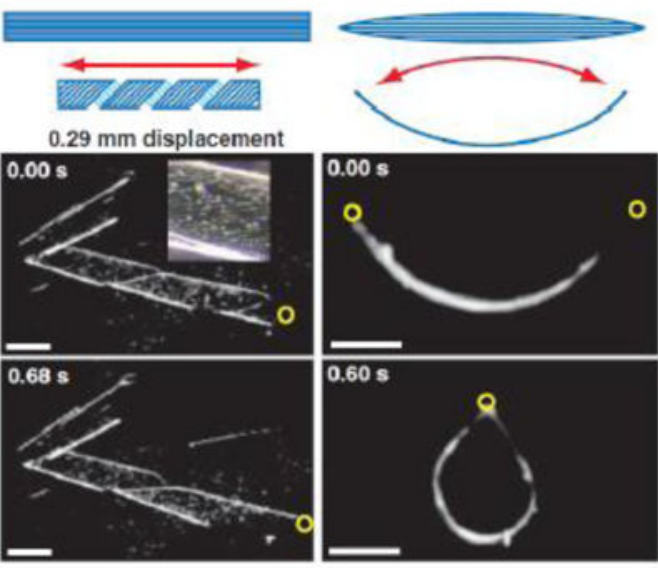

b

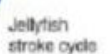
stroke oydo and thactions

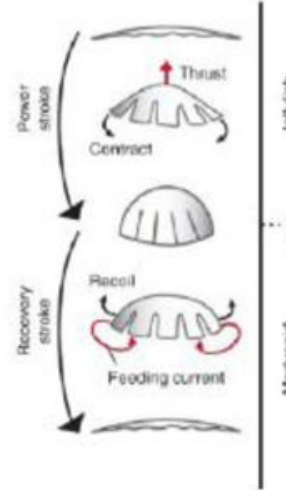

P Pacemales nstom

C

\section{ARTIFICIAL RAY}

layer 1:

3D elastomer

body

layer 2:

golden

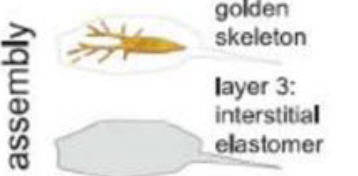

layer 4:

muscle

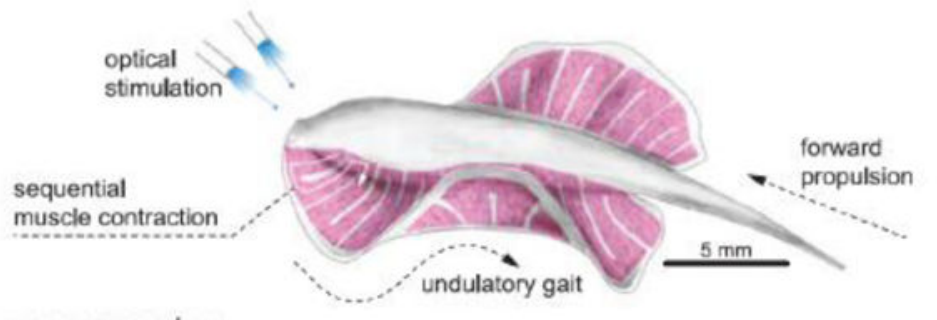

maneuvering
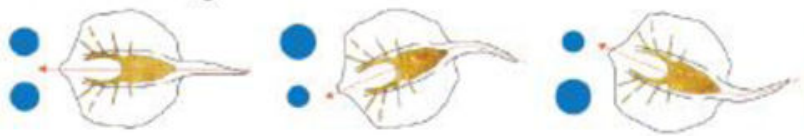

weak

strong

Figure 7. Living soft robots' motion relies on the patterning of cardiac muscle cells.

(a) Muscular thin films exploit the anisotropy of cardiomyocytes seeded onto different fibronectin-patterned PDMS surfaces to generate complex actuation patterns. Left: A strip with anisotropically organized cadiomyocytes coils and uncoils, middle: A rectangular strip with discrete arrayed muscle fibers spontaneously adopts an helical conformation, right: Cardiomyocytes aligned length-wise along a strip form a "gripper" device. ${ }^{[70]}$ (b) The locomotion of a jellyfish was replicated using electrically-stimulated muscle sheets to form a medusoid. ${ }^{[71]}$ (c) An artifical ray robot was fabricated with genetically engineered cardiomyocytes that can respond to optical stimuli. Different frequencies were used for stimulating muscle contraction and for maneuvering. ${ }^{\text {[72] }}$ 
a

Classic non-living material

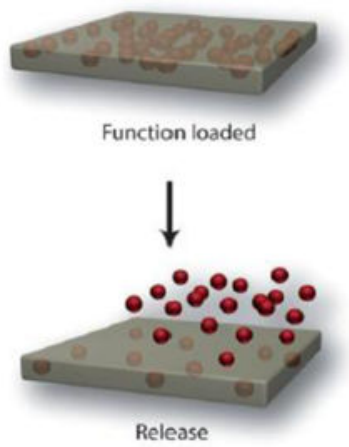

Living surface

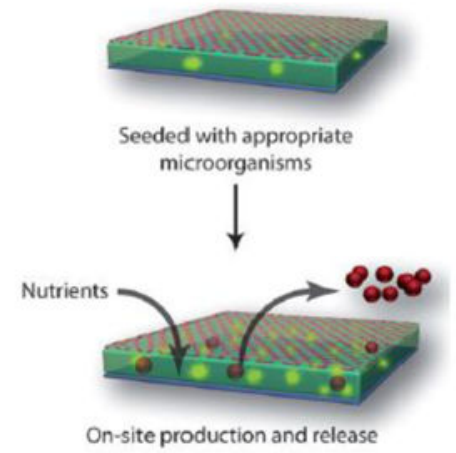

b
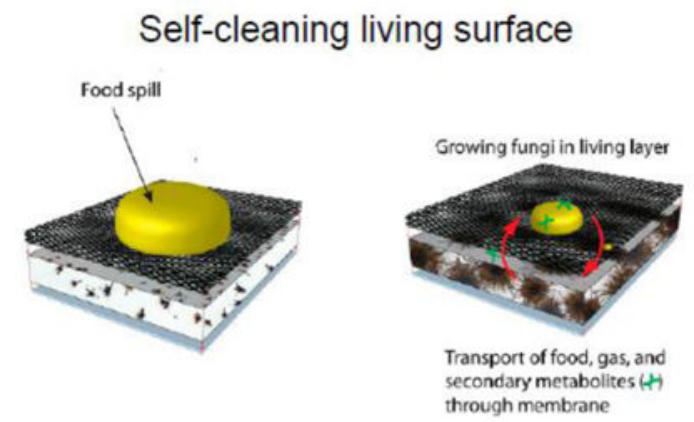

Figure 8.

Living cells have been integrated with polymers to create smart functional living polymeric composites with added functionalities. Penicillin-producing fungi cells were used to create (a) living antibiotic-releasing surfaces that can sustain release over time because of their in situ production ${ }^{[62]}$ and (b) smart self-cleaning surfaces that can metabolize food spills. ${ }^{[63]}$ 
a

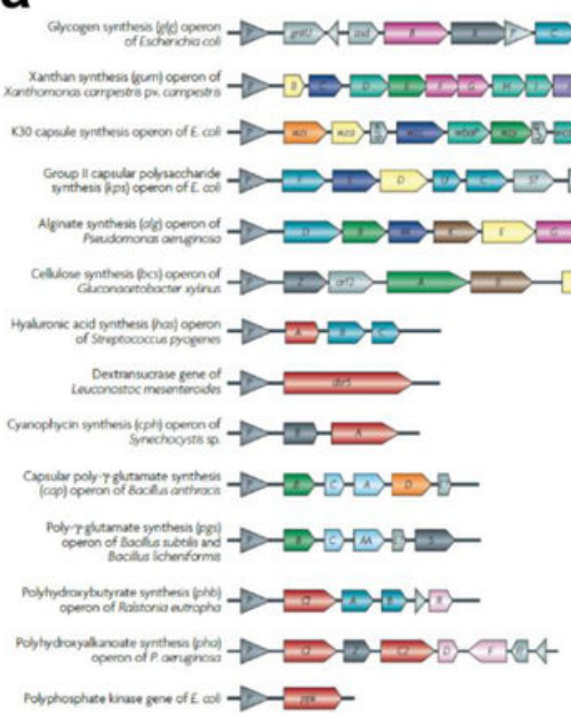

b

ins

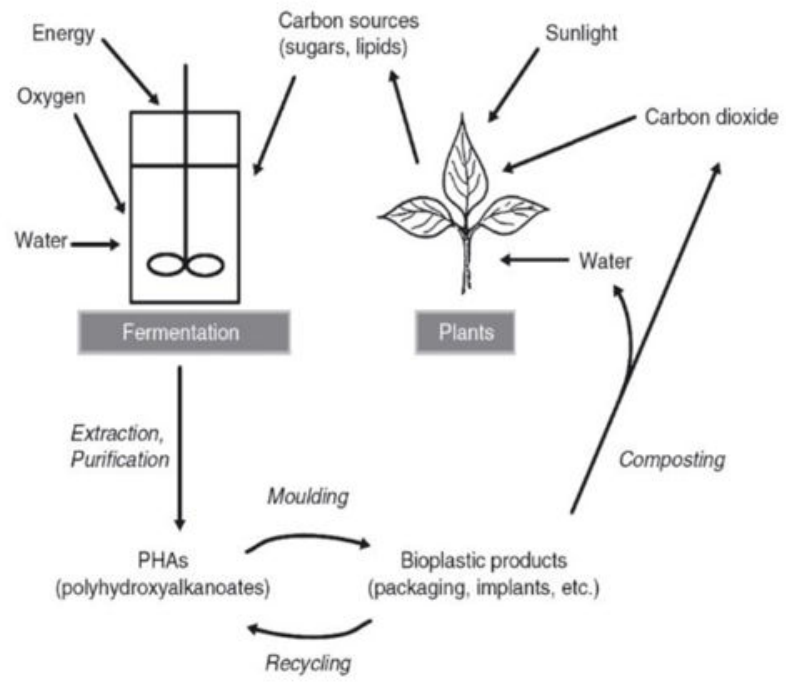

C

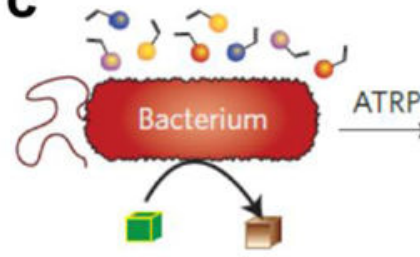

Cu reduction

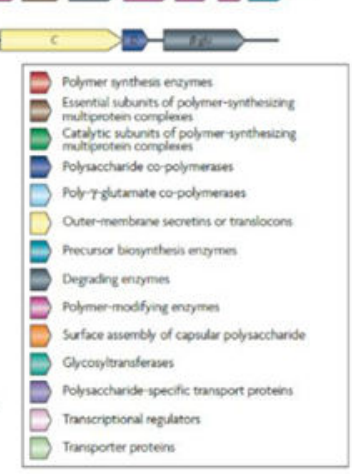

Templated
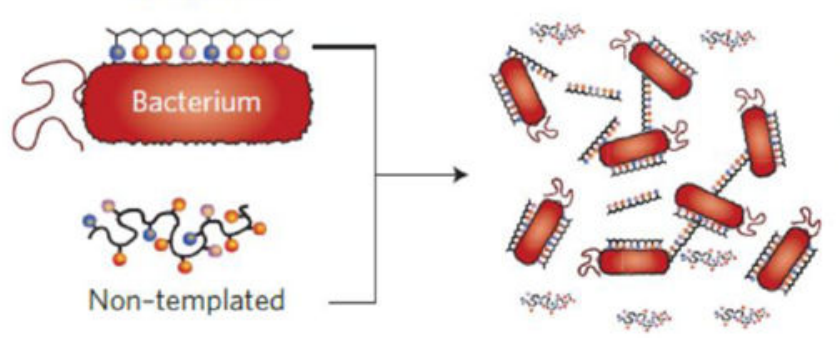

Separate polymers and cells

Figure 9. Microorganisms for the production of or the directed assembly of organic polymers. (a) Several key genes and operons are involved in the synthesis of biopolymers produced by bacteria. ${ }^{[173]}$ (b) Bacterial synthesis of polyhydroxyalkanoates through fermentation is part of the carbon life cycle of these biodegradable polymers. ${ }^{[75]}$ (c) Through their redox metabolism, bacteria can induce polymerization in monomer-catalyst suspensions, resulting in surface-bound polymers. ${ }^{[82]}$ 

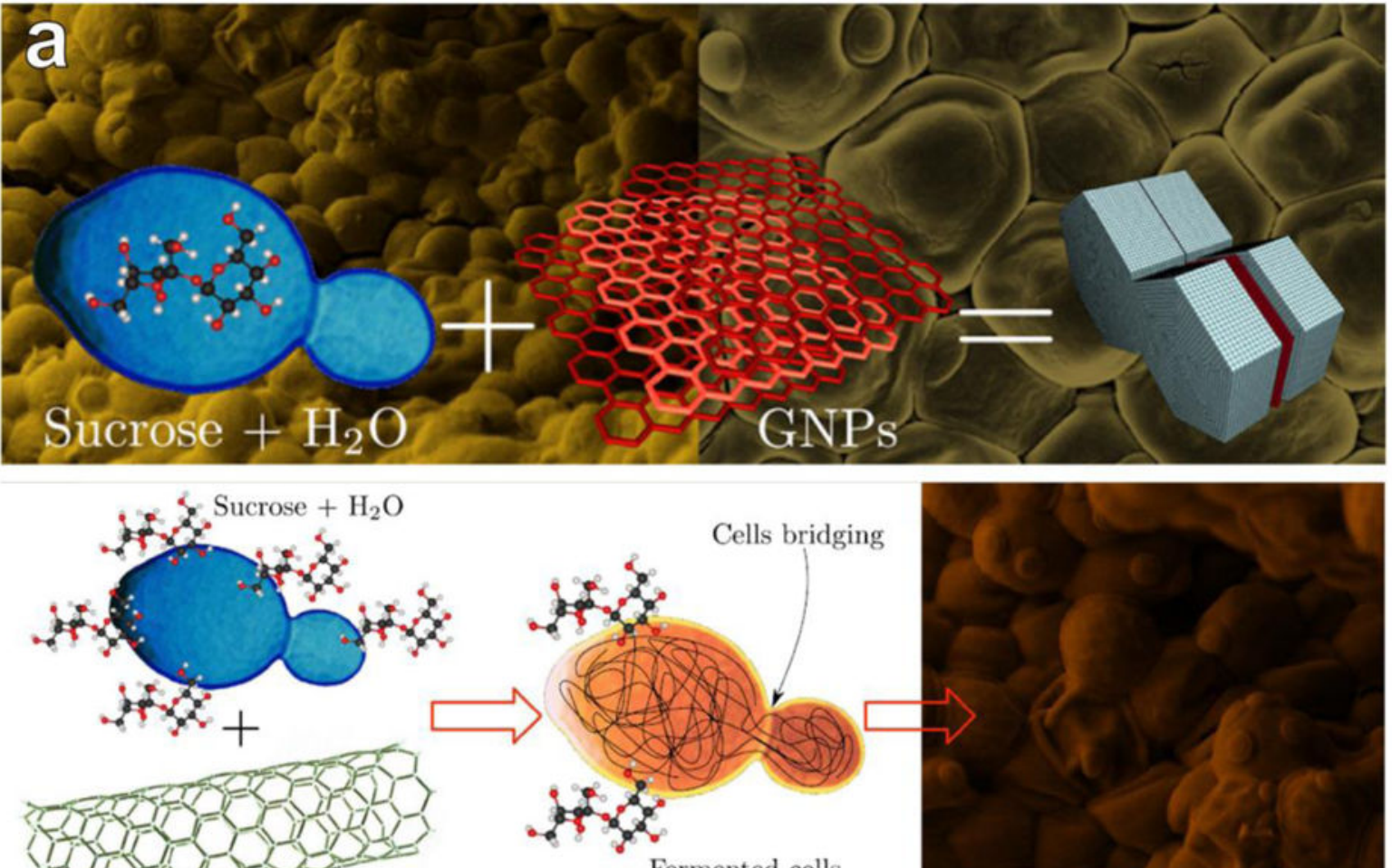

Fermented cells encapsulating CNTs

CNTs

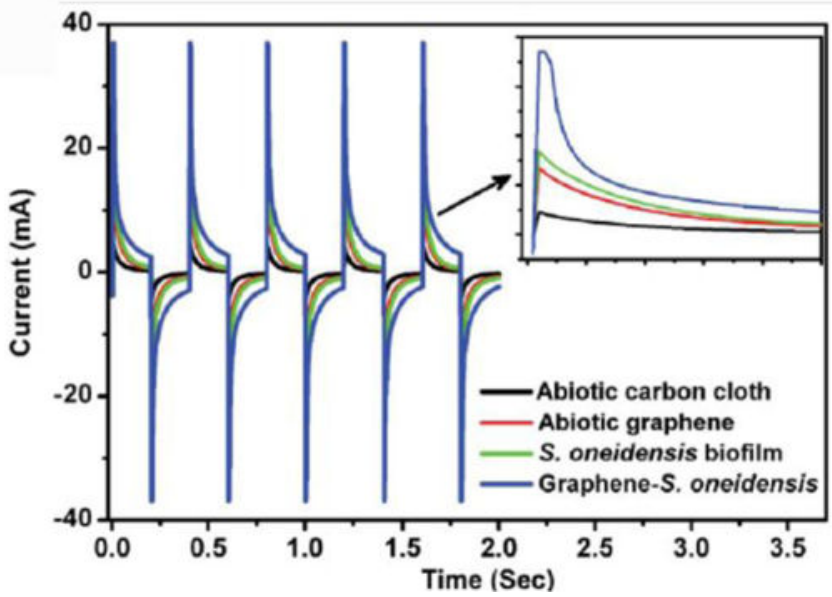

Figure 10. Living composites with carbon materials.

Integrating ELMs with inroganic materials serve for the fabrication of various devices. (a) Bionic composites made of yeast cells fermented with graphene nanoplatelets (GNPs) (top) or carbon nanotubes (CNTs) (bottom). ${ }^{[85,86]}$ (b) Biomemory device fabricated through the self-assembly of Shewanella oneidensis bacteria with graphene oxide (left: SEM image of the composite, right: Write/erase function of the biomemory device). ${ }^{[87]}$ 
a
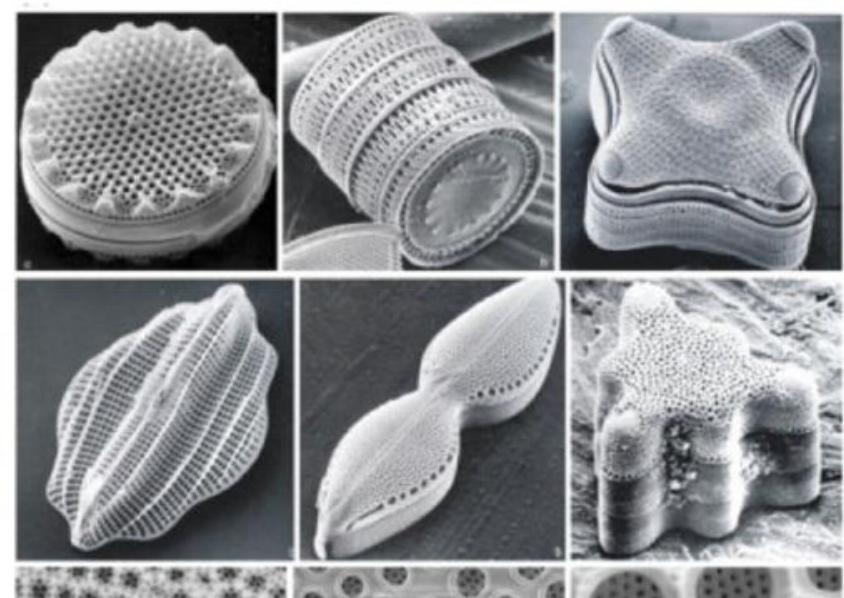

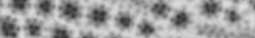

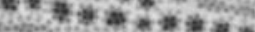

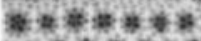

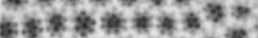

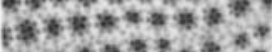

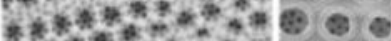

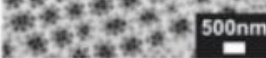
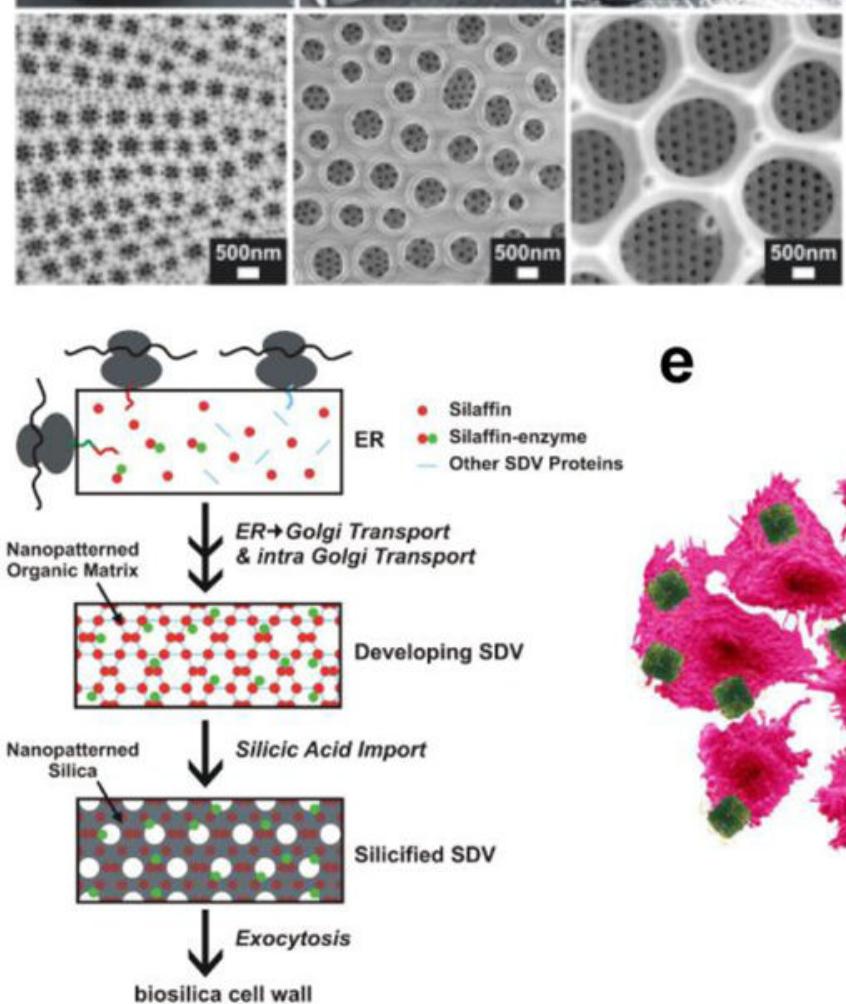

e b

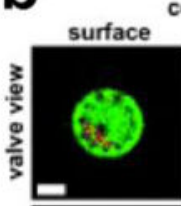

cell
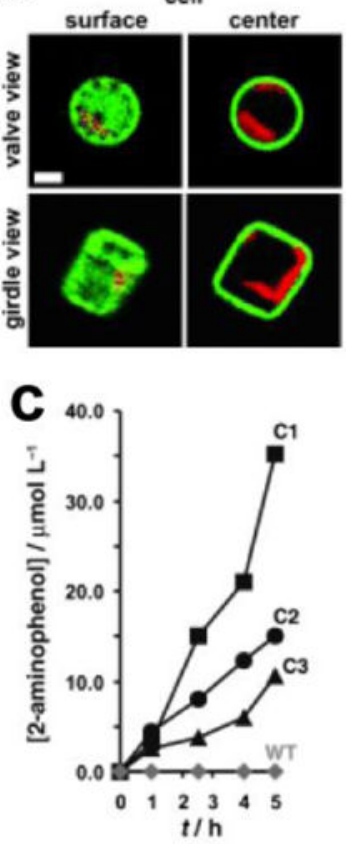

cell wall
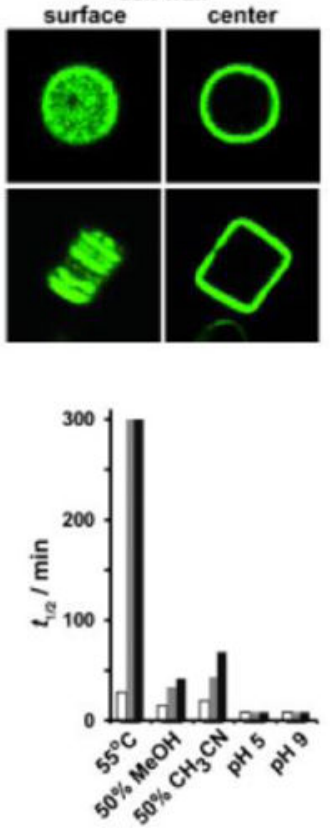
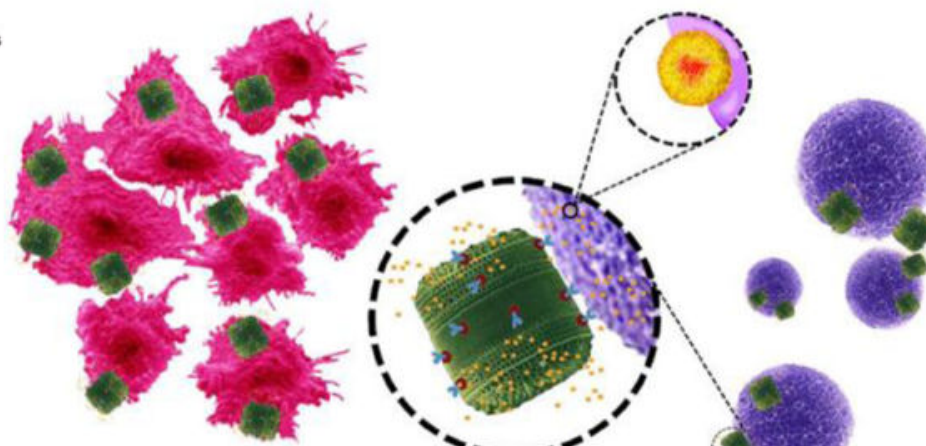

Figure 11. Silica-based ELMs from Diatom Engineering.

(a) Scanning electron microscopy images of cell walls from different diatom species. Images in top and middle rows show overviews of single silica cell walls, and images in bottom row show details of diatom silica cell walls. ${ }^{[174]}$ (b) Images obtained by confocal fluorescence microscopy of $T$. pseudonana transformants expressing silaffin fusion protein tpSil3-GFP. The four images in the left half ("cell") were taken from live cells, the images in the right half ("cell wall") were taken from isolated biosilica. Each micrograph is an overlay of two images recorded in the "green channel" (excitation: $488 \mathrm{~nm}$, emission: 505/550 nm bandpass filter) and the "red channel" (excitation: $543 \mathrm{~nm}$, emission: $585 \mathrm{~nm}$ long-pass filter). The green color is indicative of GFP, the red color is caused by chloroplast autofluorescence. Scale bar: $2 \mu \mathrm{m}$ (identical scale for all micrographs). ${ }^{[99]}$ (c) HabB activity in T. pseudonana 
biosilica. The activity was monitored by following the formation of 2-aminophenol from hydroxylaminobenzene. ${ }^{[99]}$ Line graph: Kinetics of 2-aminophenol formation by identical amounts of biosilica $(1.2 \mathrm{mg}$ ) isolated from wild-type cells (WT, gray diamonds) and transformants $\mathrm{C} 1$ (squares), $\mathrm{C} 2$ (filled circles) and C3 (triangles). Bar graph: Half-life of soluble HabB (white bars), immobilized in biosilica of transformant C1 (black bars), and immobilized in silica produced by the $\mathrm{R} 5$ peptide in vitro (gray bars). The HabB-containing samples were subjected to various treatments as indicated. ${ }^{[99]}$ (d) Hypothetical pathway for intracellular transport of silaffin-enzyme fusion proteins to the silica deposition vesicle (SDV). The fusion proteins are cotranslationally imported into the endoplasmic reticulum (ER), and the signal peptide (SP) for ER import is removed by signal peptidase. Further modifications of the fusion proteins may occur in the ER and after transport to and through the Golgi apparatus. The fusion proteins may reach the SDV via specific transport vesicles, and become incorporated into the silica-forming organic matrix in the SDV lumen. After completion of silica formation, the silaffin-enzyme fusion proteins become trapped in the deposited silica and, after exocytosis of the SDV contents, remain stably attached to the biosilica cell wall. ${ }^{[100]}$ (e) Genetically engineered diatom biosilica (green) containing liposome-encapsulated drug molecules (yellow) can be targeted to both adherent neuroblastoma cells (red) and lymphocyte cells in suspension (purple) by functionalizing the biosilica surface with cell-specific antibodies. Liposome-encapsulated drug molecules are released from the biosilica carrier in the immediate vicinity of the target cells (inset). ${ }^{[101]}$ 
a

1. Bioconcrete contains dormant bacteria.

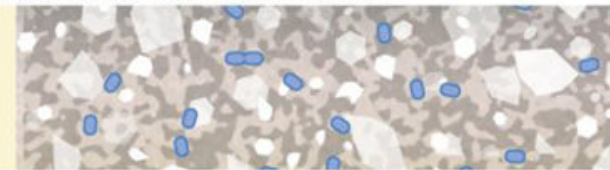

2. Upon damage, the interior is exposed to the environment.

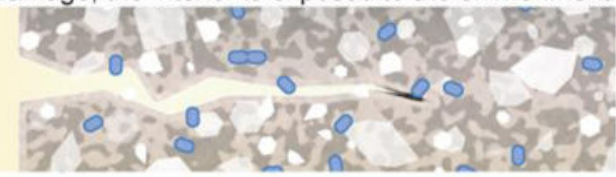

3. Water ingress activates the dormant bacteria

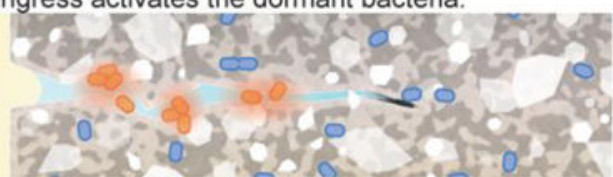

4. Bacterially induced mineralization fills the fracture

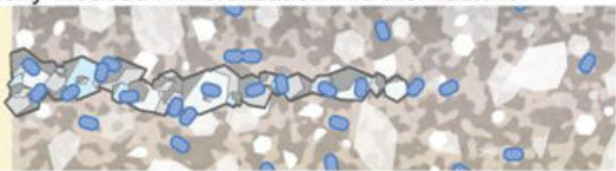

C

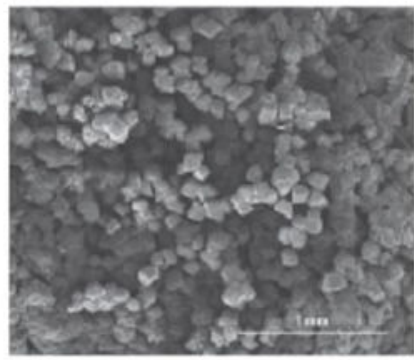

(a) $\mathrm{CaCO}_{3}$ in silica gel (100x)

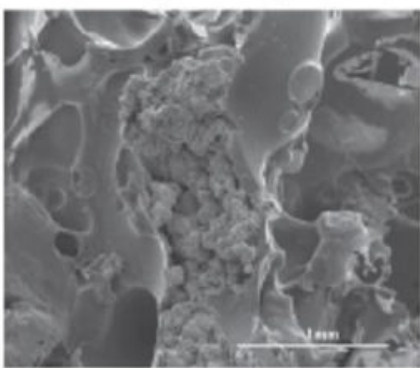

(c) $\mathrm{CaCO}_{3}$ in $\mathrm{PU}$ foam $(100 \mathrm{x})$

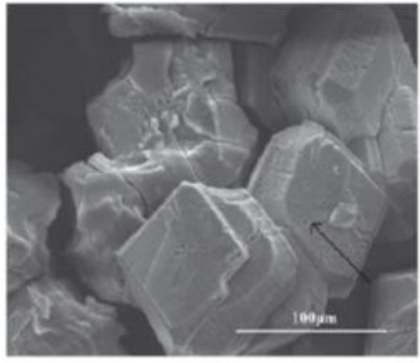

(b) $\mathrm{CaCO}_{3}$ in silica gel $(1000 \mathrm{x})$

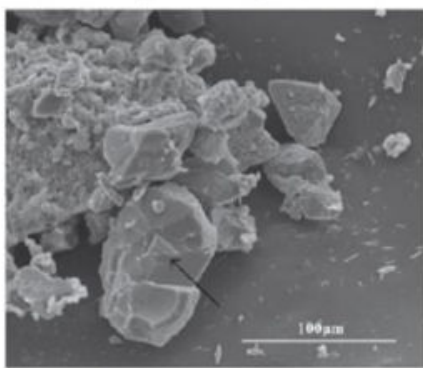

(d) $\mathrm{CaCO}_{3}$ in $\mathrm{PU}$ foam (1000x) b
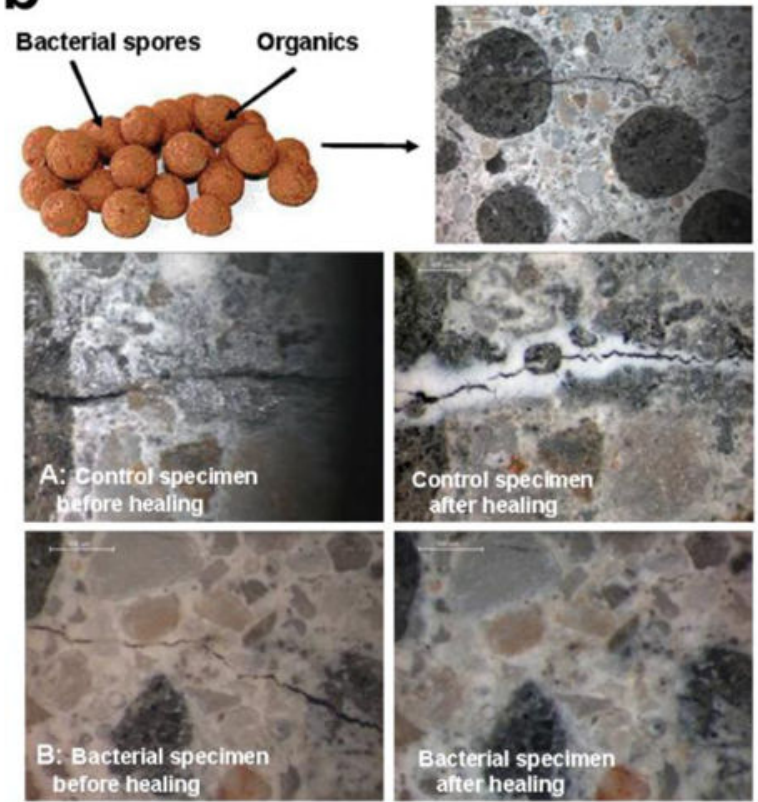

d

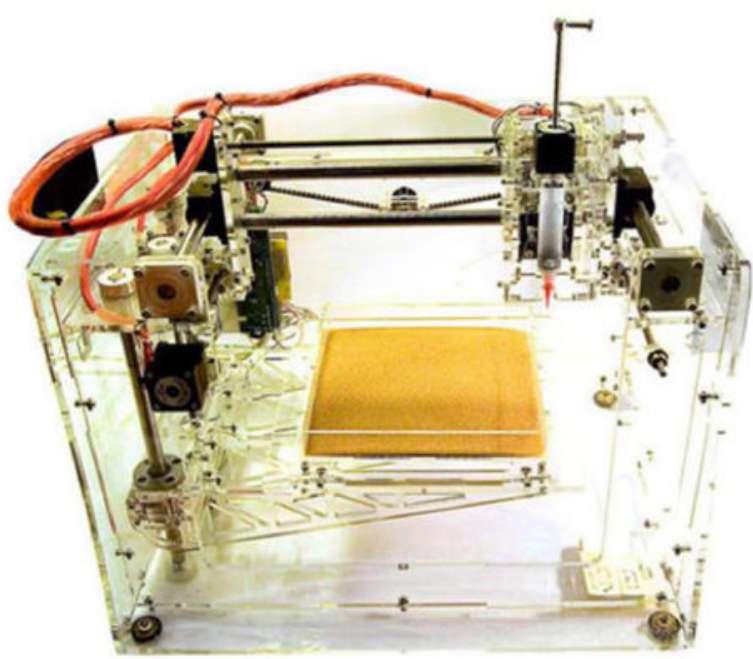

Figure 12. Self-healing Biological Concrete.

(a) Schematic of crack-healing by concrete-immobilized bacteria. Bacteria on fresh crack surfaces become activated due to water ingression, start to multiply and precipitate minerals such as calcite $\left(\mathrm{CaCO}_{3}\right)$, which eventually seal the crack ${ }^{[103]}$ (b) Self-healing admixture composed of expanded clay particles (left) loaded with bacterial spores and organic biomineral precursor compound (calcium lactate). When embedded in the concrete matrix (right) the clay particles serve as internal reservoirs containing the two-component healing agent consisting of bacterial spores and a suitable bio-mineral precursor compound. ${ }^{[102]}$ 
Light microscopic images (40x magnification) of pre-cracked control (A) and bacterial (B) concrete specimen before (left) and after (right) healing following 2 weeks of submersion in water. Mineral precipitation occurred predominantly near the crack rim in control but inside the crack in bacterial specimens. ${ }^{[102]}$ (c) Scanning electron micrographs of $\mathrm{CaCO}_{3}$ precipitation in the silica gel and PU foam. ${ }^{[105]}$ (d) bioMASON machine for producing biobricks. ${ }^{[175]}$ 
a

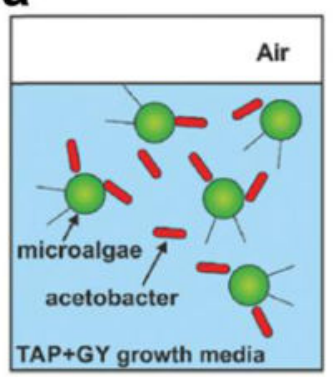

b

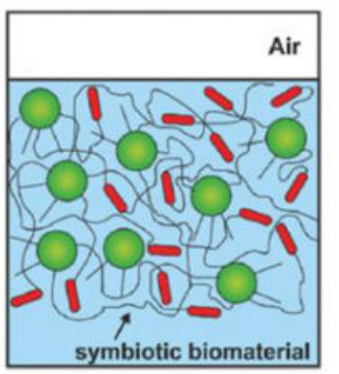

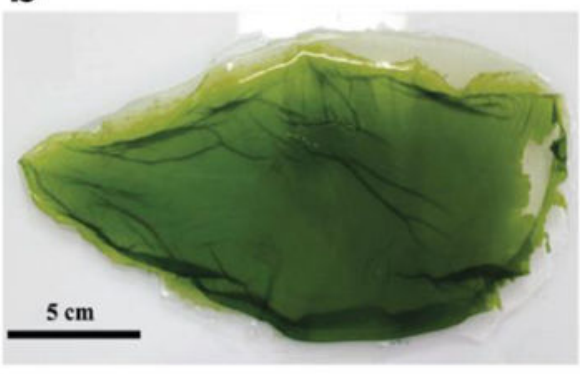

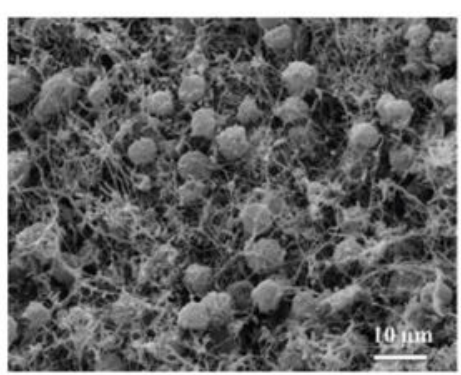

Figure 13. Engineered ELMs from Mutualistic Microbial Consortia.

A collection of microorganisms with mutualistic or complementary functions can be assembled to produce functional materials. (a) Living soft matter was produced in situ by growing cellulose-producing bacteria and photosynthetic microalgae. ${ }^{[110]}$ (b) The resulting material consists of a moldable gel of entrapped microalgae in a cellulose matrix. 

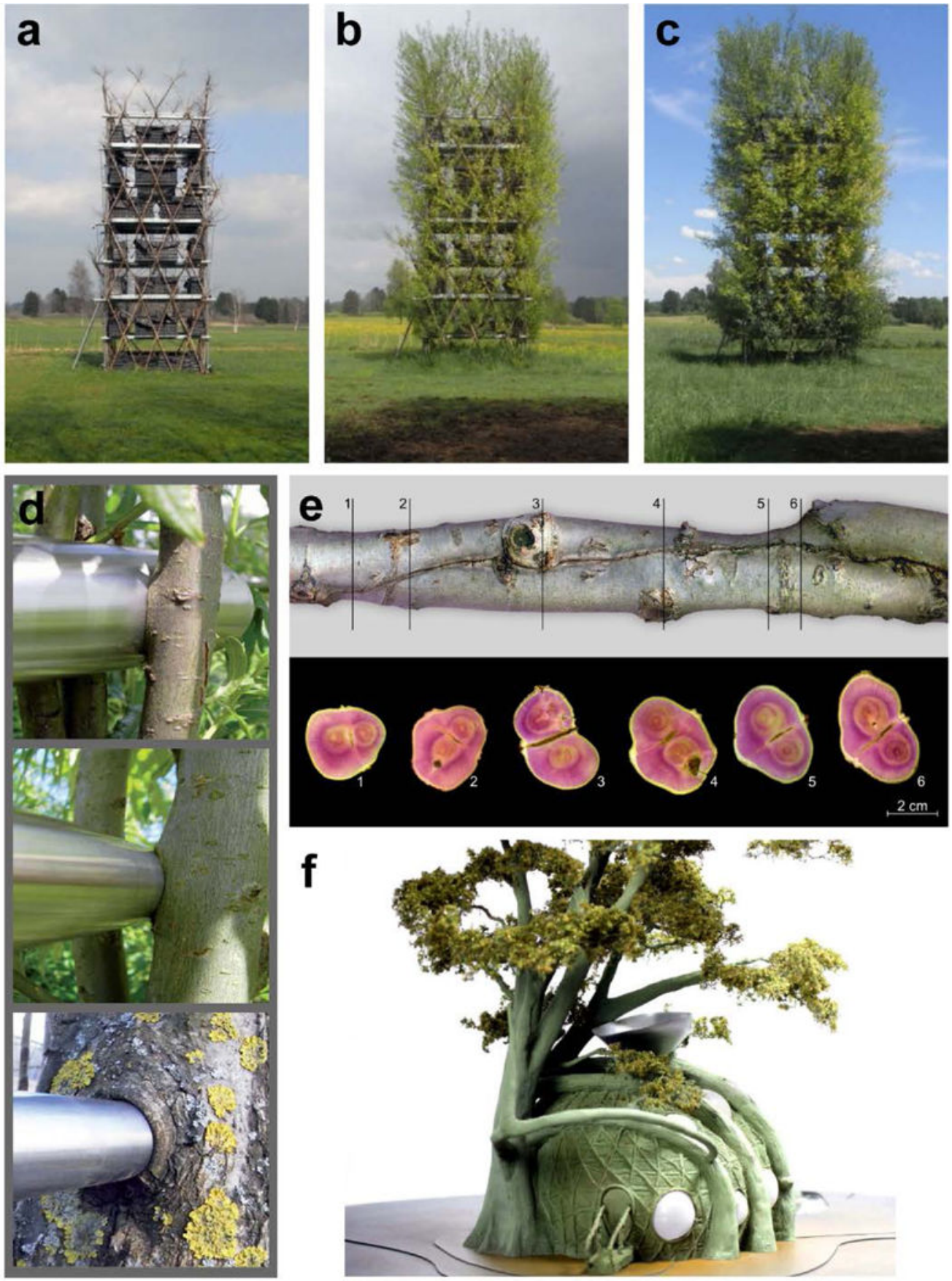

Figure 14. Lignocellulosic Living Organisms as ELMs.

(a-c) Tree shaping allows for the mechanical manipulation of living tree tissues into a growing structure. (d) One version of this architectural design system is known as Baubontanik and incorporates modern design materials with tree shaping, as shown in the construction of a tower. Over time, the trees are able to fuse around temporary metal scaffolding. (e) Tree shaping takes advantage of inosculation, or proximity grafting, the ability of tree tissues in trunks, branches, or roots to fuse together when in close proximity. Shown are cross-sections of inosculated branches. All photos for (a-e) copyright Ferdinand 
Ludwig. (f) Concept designs of a tree-based habitat in which the tree is shaped using CNC scaffolds. Photo copyright Mitchell Joachim. 

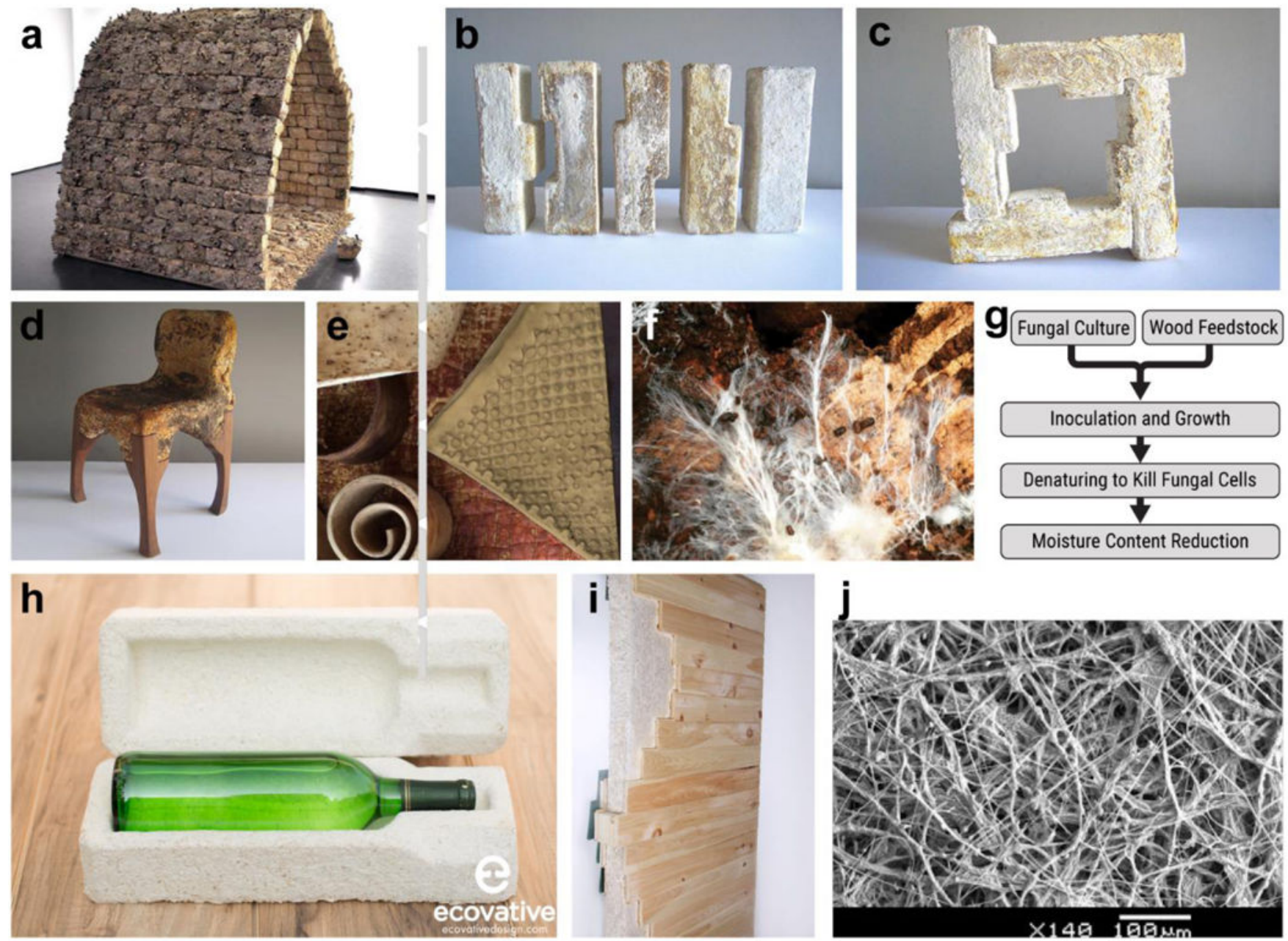

Figure 15. Mycelium-based ELM materials.

(a) A 'mycotecture' concept structure composed of mycelium, Mycotectural Alpha, on exhibit at Kunsthalle Düsseldorf in 2009. Photograph copyright Philip Ross. (b) Myceliumbased bricks for construction. (c) When the bricks are placed together, the mycelium grow into each other, organically fusing the bricks together. (d) The mycelial material can also be shaped into functional structures such as furniture. (e) In addition, flexible materials such as tough leather-like fabrics can be made from mycelium. (f) A photograph of Mycoworks mycelium material shows the wood chip feedstock and the growing mycelial hyphae. Photos (a-f) are all copyright MycoWorks. (g) A flowchart of the process for preparation of mycelial materials. Mycelium-based foam technology from Ecovative Design used for ecologically-friendly packaging (h) and building insulation (i). (j) SEM image of the mycelium from the Ecovative materials. Photos (h-j) copyright Ecovative Design. 

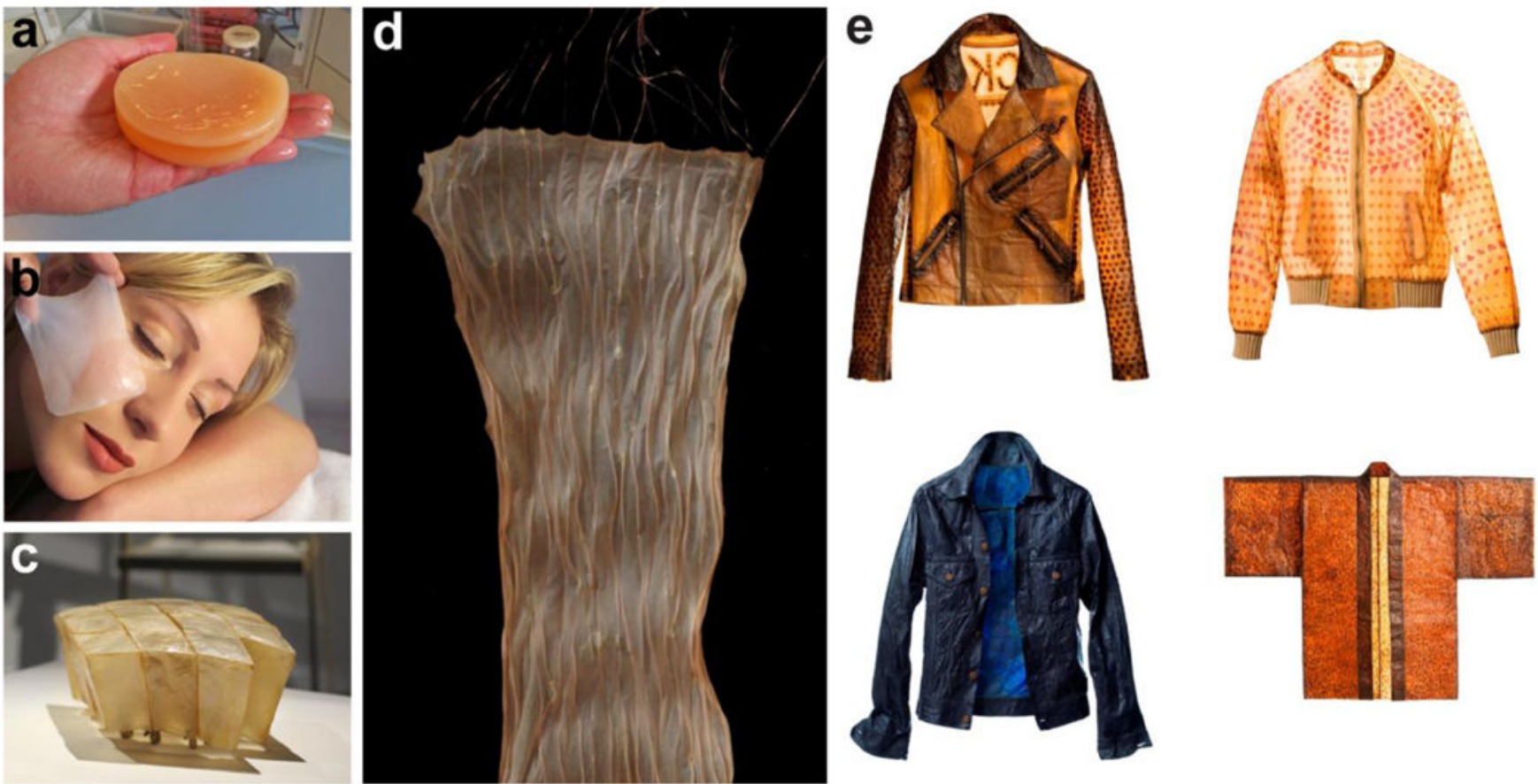

Figure 16. Large-scale production of bacterial cellulose materials.

(a) Harvested bacterial cellulose material. Photo copyright Scobytec. (b) Skin dressing made from microbial cellulose materials. Photo copyright www.fzmb.de. (c) As a structural material, modular geometric objects can be created from molded bacterial cellulose. Photo copyright Jannis Hülsen. (d) An example of architectural materials made of bacterial cellulose grown on a support scaffold of jute wires. Photo copyright Institute for Advanced Architecture of Catalonia. (e) Bacterial cellulose can also be used as a large-scale textile material, here used to create various garments. Photo copyright Suzanne Lee. 

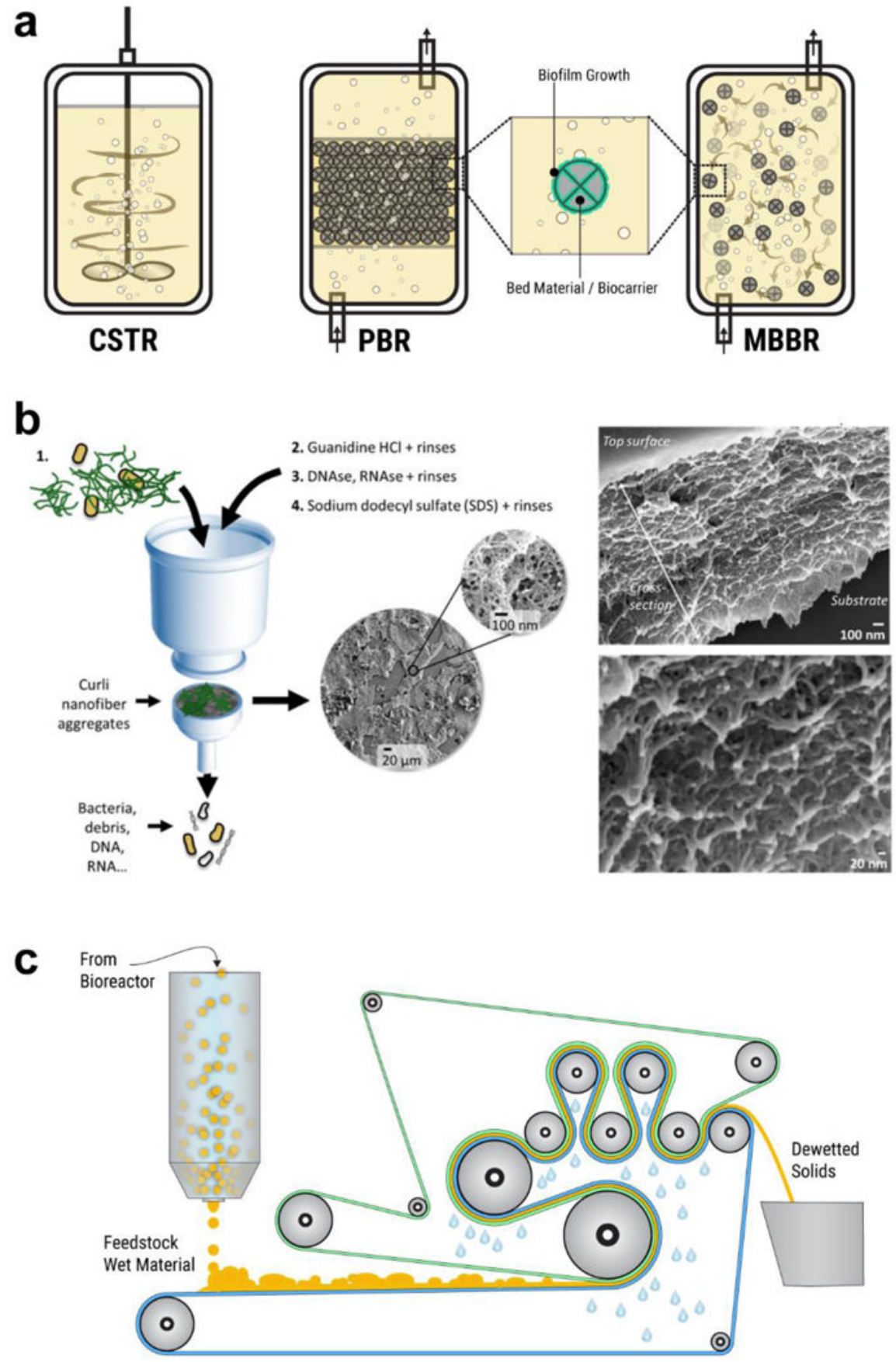

Figure 17. Large-scale Bioproduction Technologies for ELMs.

(a) Basic bioreactor architectures. In contrast to CSTR reactors, which are efficient for production of planktonic cells, PBR and MBBRs contain packing materials that allow for the growth of interfacial biomass and are more suited for maximizing biofilm growth. (b) Preparatory-scale ELMs can be separated from the cellular components after growth, using chemical treatments and vacuum-assisted filtration. Right images are SEM micrographs showing isolated layered ELM amyloid mat materials. (c) On an industrial level, highthroughput separation of solids from liquids can be applied to ELMs grown in liquid 
bioreactors using belt press filters, which sandwiches the material to be processed between two membranes of defined pore size and uses pressure from rollers to remove liquids, producing solids that can be processed further. 

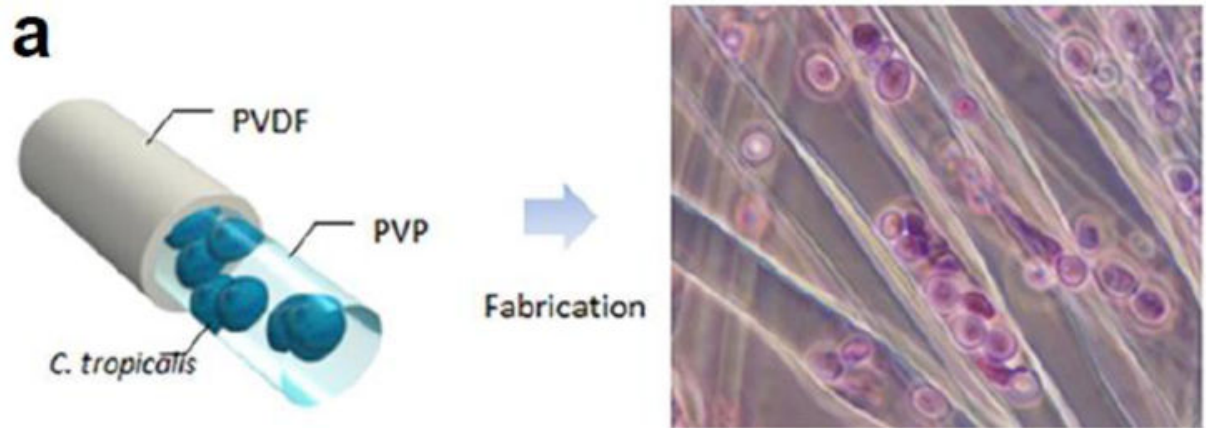

Application
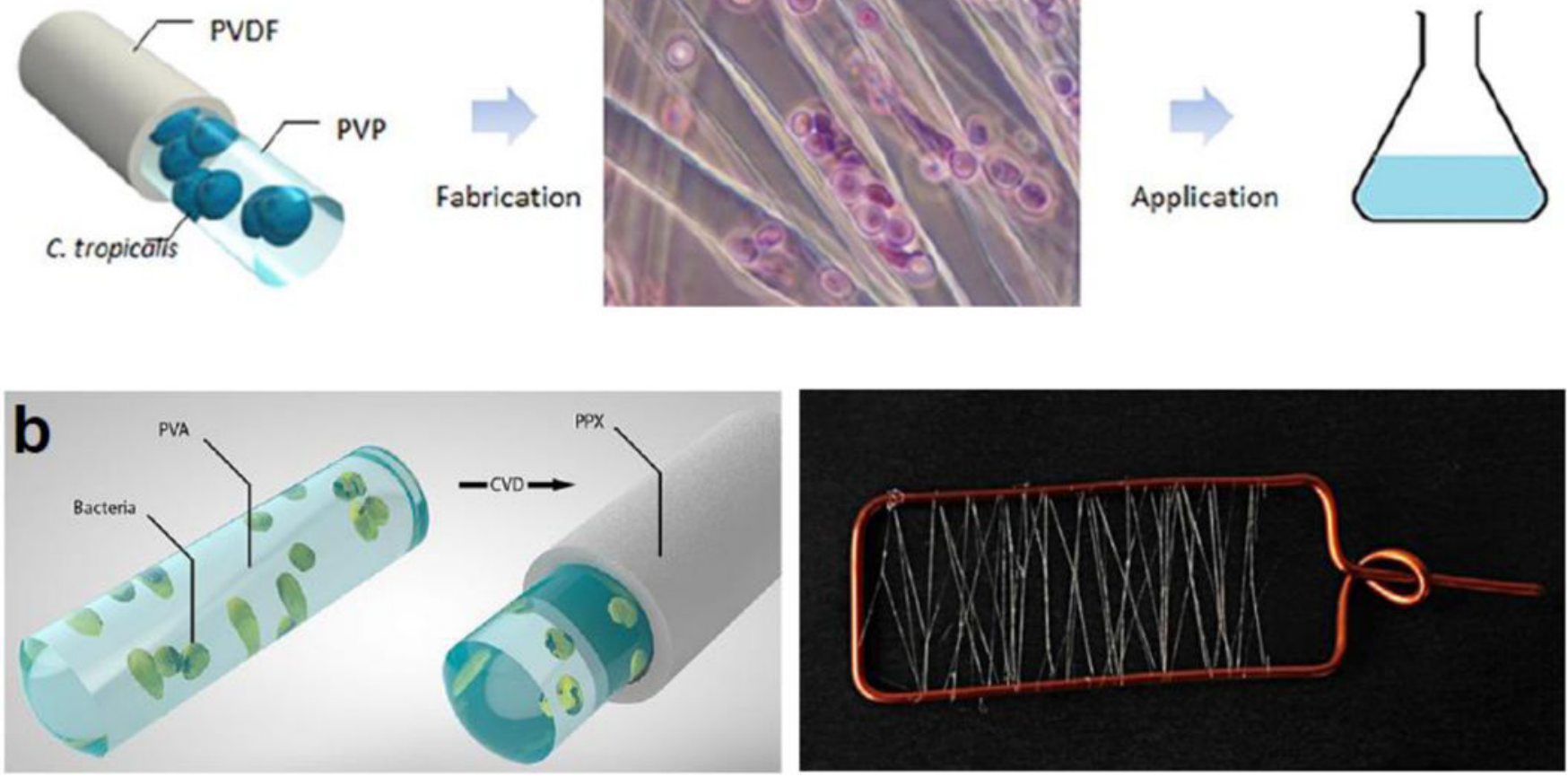

Figure 18. Electrospun living polymer fibers.

(a) Yeast cells were embedded in a water-soluble polyvinylpyrrolidone (PVP) core matrix, and enveloped with a poly vinylidene fluoride-cohexafluoropropylene shell. In the middle panel, encapsulated yeast cells are shown in purple. ${ }^{[146]}$ (b) Electrospun living bacteria composite fibers were prepared using a poly-(vinyl alcohol) (PVA) core subsequently coated with a hydrophobic poly(p-xylylene) (PPX) shell via chemical vapor deposition (left). The fibers were mounted onto a wire frame $(5 \mathrm{~cm}$ wide) that could be used for decontamination $(\text { right })^{[147]}$ 

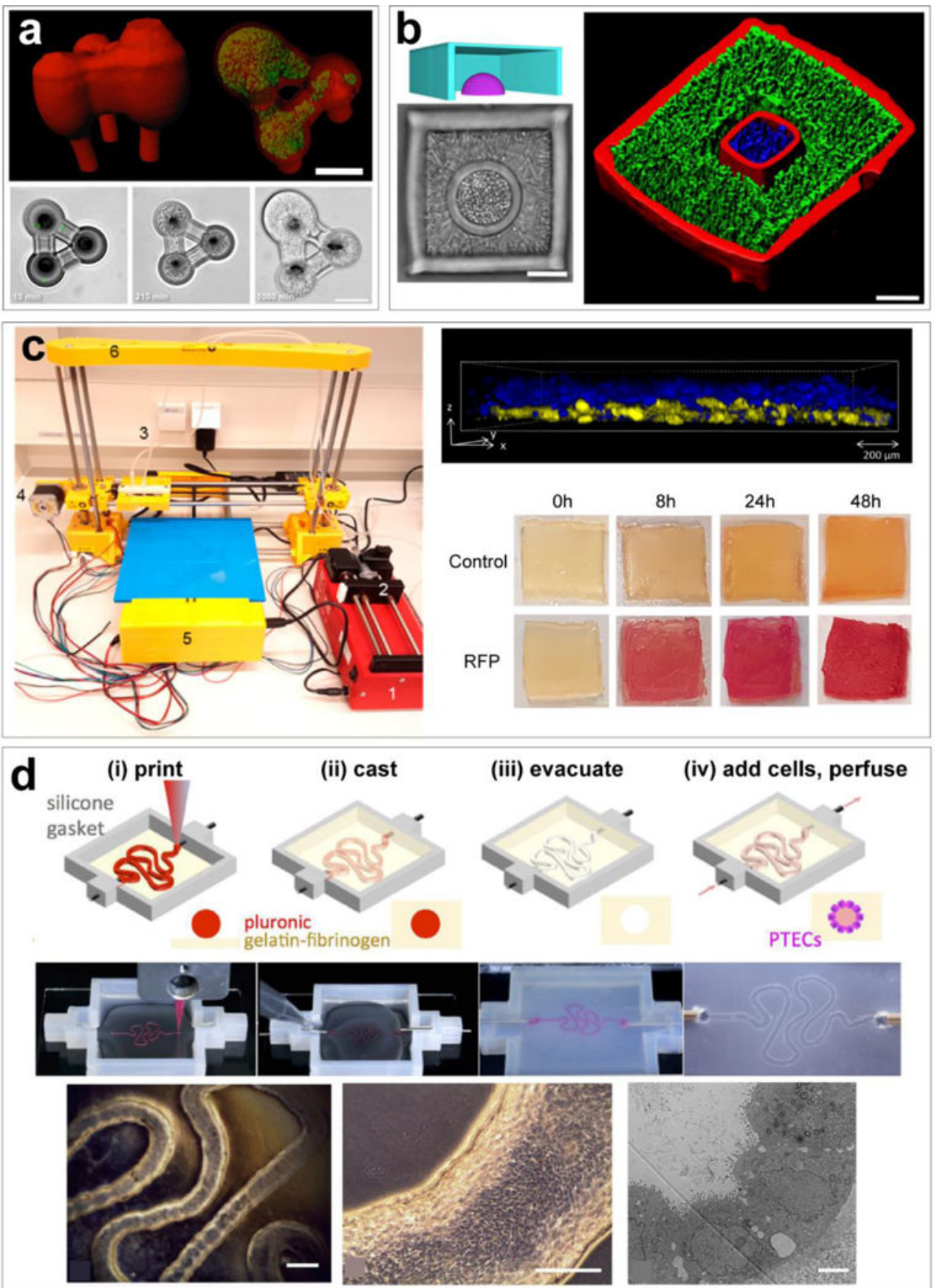

Figure 19. 3D Printing for Spatial Patterning of ELMs.

(a) Top: Confocal laser scanning microscopy (CLSM) reconstruction of bacteria (green) within spheroid "rooms" created from photocrosslinked gelatin that are connected by channels $(r e d)$. Scale bar, $20 \mu \mathrm{m}$. Bottom: Light microscopy top view of encapsulated $P$. aeruginosa cells growing within the microfabricated structure over time. Scale bar, $20 \mu \mathrm{m}$. (b) This method can be used to construct complex structures containing different bacterial species, as demonstrated from nested structures where the inner chamber contains $S$. aureus bacteria surrounded by $P$. aeruginosa bacteria in the outer chamber. Left: Model and light 
microscopy image of nested rooms. Right, top: CLSM reconstruction cross-section view of the structure. Scale bars, $10 \mu \mathrm{m}$. All images in (a-b) from the Shear group. ${ }^{[149]}$ (c) Left: An inexpensive bacterial 3D printer using a modified commercial kit integrated with a syringe pump and alginate-based bioinks. Right: Top shows a CLSM reconstruction of two E. coli populations expressing different fluorescent proteins in different 3D-printed alginate layers. Bottom shows rhamnose-induced expression of RFP from 3D-printed structures, demonstrating viability. ${ }^{[150]}$ (d) Top: schematic and photographs of the 3D tissue-printing process using fugitive ink from the Lewis lab, here used to fabricate a structure simulating kidney nephron tubules ${ }^{[151]}$. Bottom: After seeding with proximal tubule epithelial cells, shear stress from flow through the printed tubule induces the formation of ECM and functionalization of the cells, forming a polarized tissues that structurally and functionally recapitulate aspects of kidney epithelium. (left to right) Phase contrast images of cells in 3Dprinted tubule after 6 weeks, scale bars at 500 and $250 \mu \mathrm{m}$. TEM cross-section after 5 weeks, scale bar is $5 \mu \mathrm{m}$. 

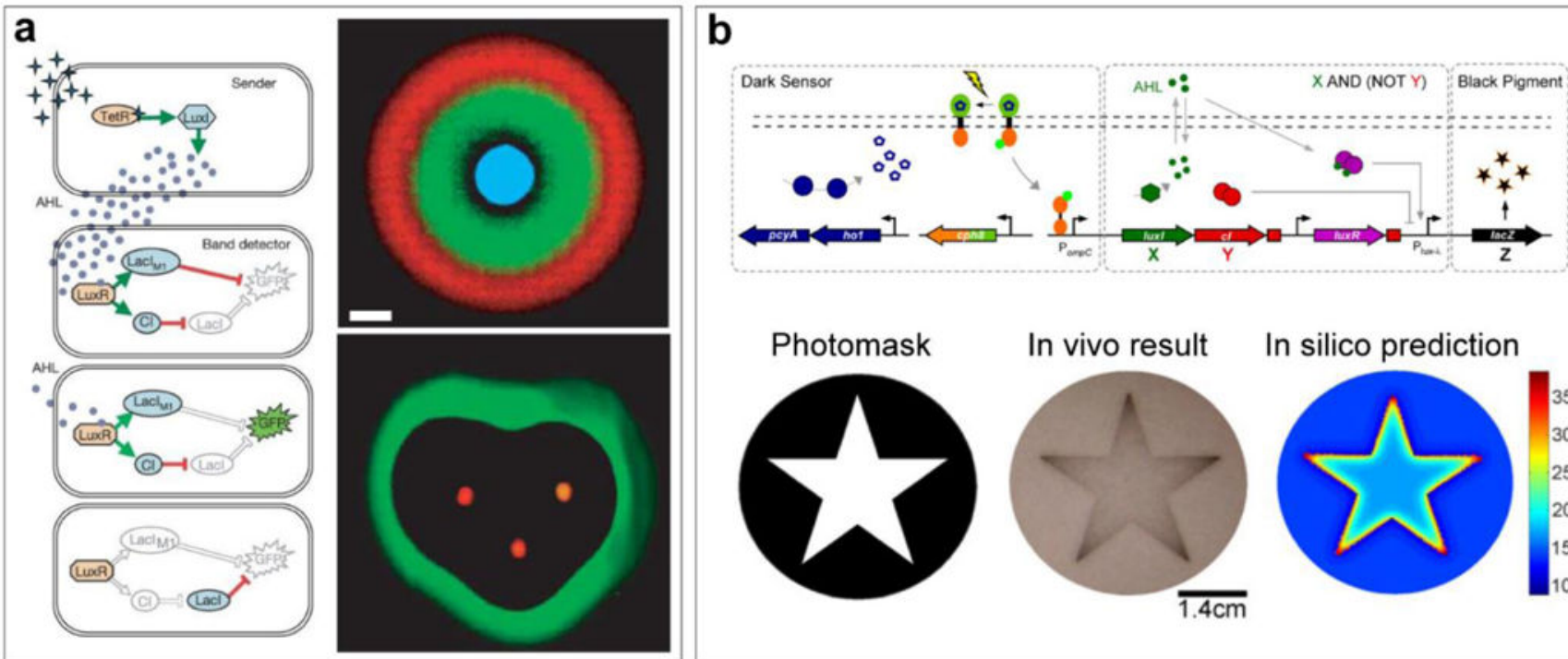

C : AHth

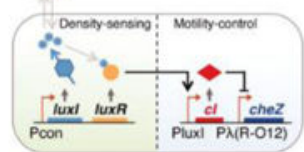

Stop Move
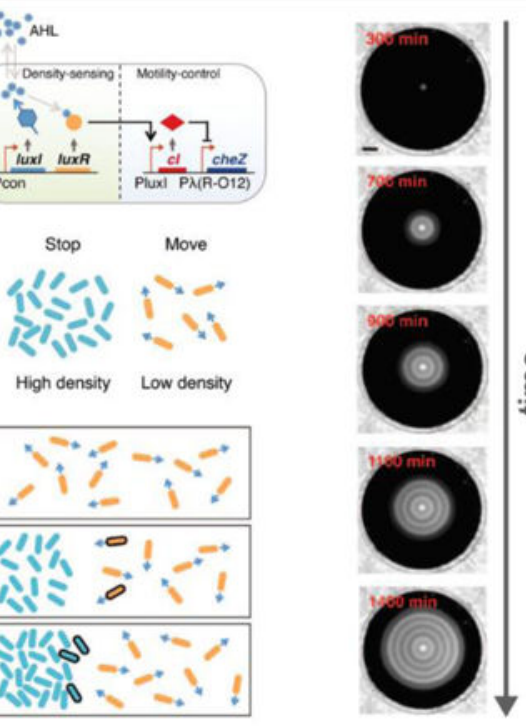

d
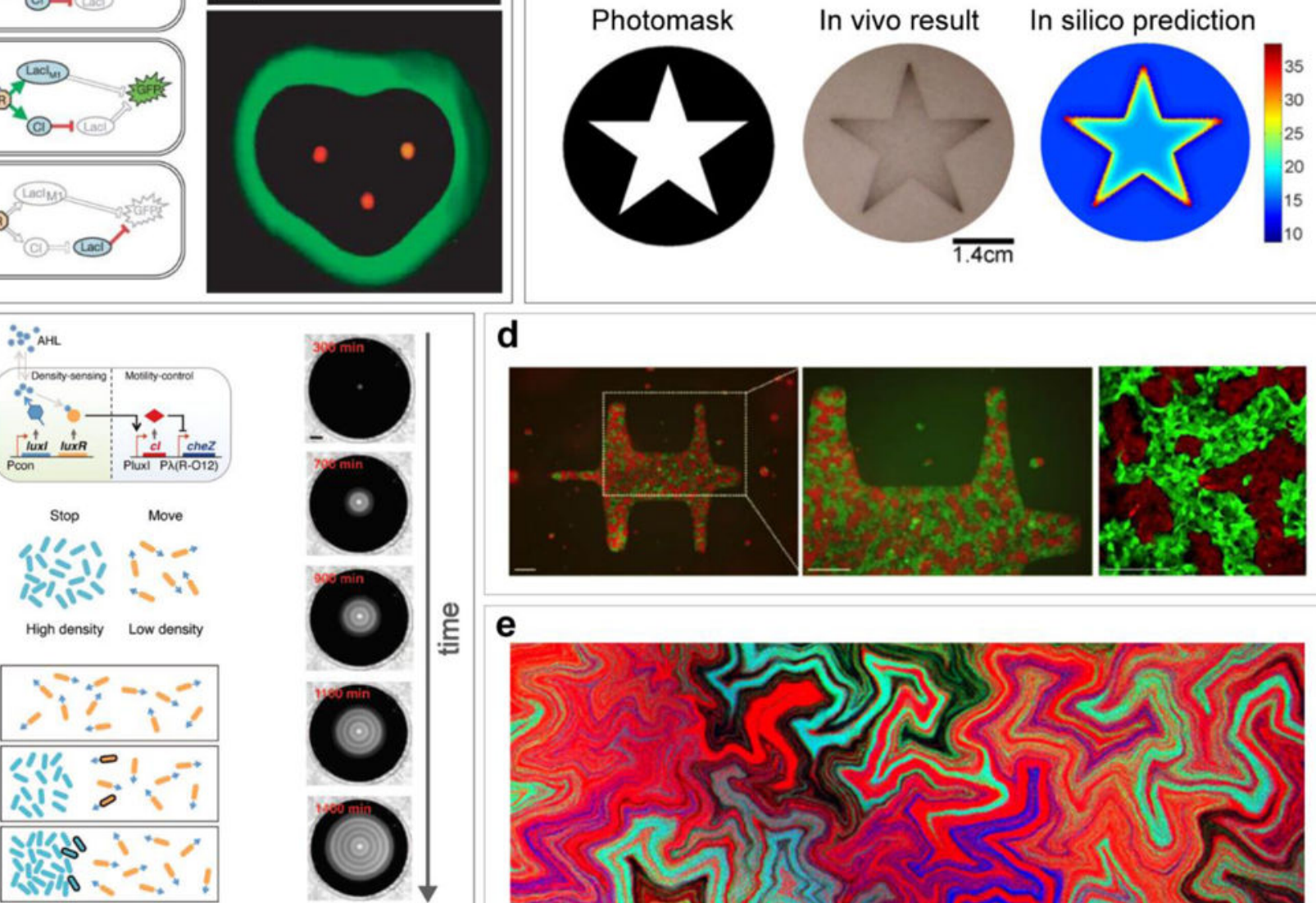

e

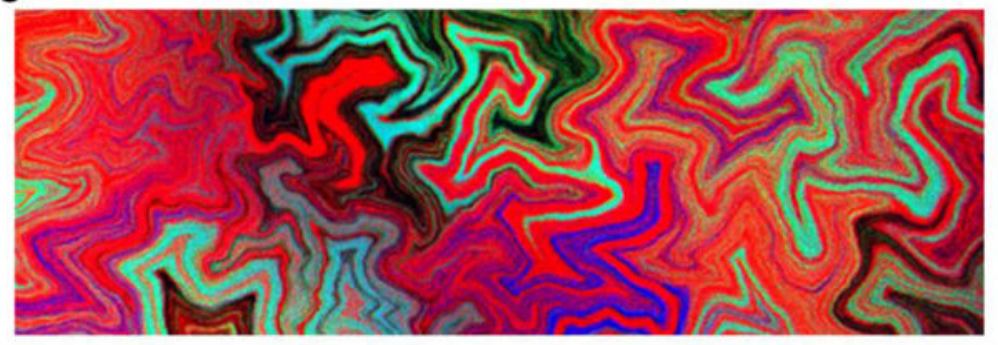

Figure 20. Synthetic Morphogenesis for Engineering Hierarchically Defined Multicellular Structures.

(a) Use of AHL production and sensing elements to generate a band detector circuit for genetically programmed morphogenesis. Right, top image shows a bullseye pattern formed by the sender cells in the middle expressing CFP surrounded by two cell types with different band detectors, expressing GFP and DsRed. Right, bottom image shows designed shapes can be generated by defined placement of the sender cells (here expressing DsRed); the GFP cells containing the band detector circuit only grow at a defined AHL range and thus form a heart shape. ${ }^{[161]}$ (b) By combining a genetic dark sensor with an AHL-gradient response circuit, only cells directly adjacent to the dark zones (edges) are turned on by the synthetic circuit, creating an edge-detection device. Bottom images: left shows the photomask used in the experiment, center is a photo of a bacterial lawn containing the edge detector circuit, and right shows the mathematically modeled prediction. ${ }^{[162]}$ (c) Generation of striped patterns from growing populations of bacterial cells using an AHL-generation circuit that stops 
cellular motility at high cell densities. As cells swarm outward, they produce concentric bands containing nonmotile cells. The stripe periodicity can be tuned genetically. ${ }^{[163]}$ (d) Differential adhesion-based engineering of multicellular patterning, using two populations of mammalian cells expressing two different pairs of cadherins with GFP or mCherry as markers. Epifluorescence microscopy images (left, center) showing the boundary zones. Right, zoom-in confocal fluourescence image. All scale bars are 200 microns. ${ }^{[165]}$ (e) Confocal microscopy image of $B$. subtilis biofilm populations expressing different fluorescent proteins that self-organize into fractal patterns. Image from University of Cambridge, Fractal Bacteria: Predictably Beautiful 3, by Fernan Federici, PJ Steiner, Tim Rudge, and Jim Haseloff. 